\title{
Predictions of covariant chiral perturbation theory for nucleon polarisabilities and polarised Compton scattering
}

\author{
Vadim Lensky ${ }^{1,2,3,4, a}$, Judith A. McGovern ${ }^{4}$, Vladimir Pascalutsa ${ }^{1}$ \\ ${ }^{1}$ Institut für Kernphysik and PRISMA Cluster of Excellence, Johannes Gutenberg Universität Mainz, 55128 Mainz, Germany \\ ${ }^{2}$ Institute for Theoretical and Experimental Physics, 117218 Moscow, Russia \\ ${ }^{3}$ National Research Nuclear University MEPhI (Moscow Engineering Physics Institute), 115409 Moscow, Russia \\ ${ }^{4}$ Theoretical Physics Group, School of Physics and Astronomy, University of Manchester, Manchester M13 9PL, UK
}

Received: 16 October 2015 / Accepted: 10 November 2015 / Published online: 19 December 2015

(C) The Author(s) 2015. This article is published with open access at Springerlink.com

\begin{abstract}
We update the predictions of the SU(2) baryon chiral perturbation theory for the dipole polarisabilities of the proton, $\left\{\alpha_{E 1}, \beta_{M 1}\right\}_{p}=\{11.2(0.7), 3.9(0.7)\} \times 10^{-4} \mathrm{fm}^{3}$, and obtain the corresponding predictions for the quadrupole, dispersive, and spin polarisabilities: $\left\{\alpha_{E 2}, \beta_{M 2}\right\}_{p}$ $=\{17.3(3.9),-15.5(3.5)\} \times 10^{-4} \mathrm{fm}^{5},\left\{\alpha_{E 1 v}, \beta_{M 1 v}\right\}_{p}$ $=\{-1.3(1.0), 7.1(2.5)\} \times 10^{-4} \mathrm{fm}^{5}$, and $\left\{\gamma_{E 1 E 1}, \gamma_{M 1 M 1}\right.$, $\left.\gamma_{E 1 M 2}, \gamma_{M 1 E 2}\right\}_{p}=\{-3.3(0.8), 2.9(1.5), 0.2(0.2), 1.1$ $(0.3)\} \times 10^{-4} \mathrm{fm}^{4}$. The results for the scalar polarisabilities are in significant disagreement with semi-empirical analyses based on dispersion relations; however, the results for the spin polarisabilities agree remarkably well. Results for proton Compton-scattering multipoles and polarised observables up to the Delta(1232) resonance region are presented too. The asymmetries $\Sigma_{3}$ and $\Sigma_{2 x}$ reproduce the experimental data from LEGS and MAMI. Results for $\Sigma_{2 z}$ agree with a recent sum rule evaluation in the forward kinematics. The asymmetry $\Sigma_{1 z}$ near the pion production threshold shows a large sensitivity to chiral dynamics, but no data is available for this observable. We also provide the predictions for the polarisabilities of the neutron, the numerical values being $\left\{\alpha_{E 1}, \beta_{M 1}\right\}_{n}=\{13.7(3.1), 4.6(2.7)\} \times$ $10^{-4} \mathrm{fm}^{3},\left\{\alpha_{E 2}, \beta_{M 2}\right\}_{n}=\{16.2(3.7),-15.8(3.6)\} \times$ $10^{-4} \mathrm{fm}^{5},\left\{\alpha_{E 1 v}, \beta_{M 1 v}\right\}_{n}=\{0.1(1.0), 7.2(2.5)\} \times 10^{-4}$ $\mathrm{fm}^{5}$, and $\left\{\gamma_{E 1 E 1}, \gamma_{M 1 M 1}, \gamma_{E 1 M 2}, \gamma_{M 1 E 2}\right\}_{n}=\{-4.7(1.1)$, $2.9(1.5), 0.2(0.2), 1.6(0.4)\} \times 10^{-4} \mathrm{fm}^{4}$. The neutron dynamical polarisabilities and multipoles are examined too. We also discuss subtleties related to matching the dynamical and static polarisabilities.
\end{abstract}

\footnotetext{
a e-mail: lensky@itep.ru
}

\section{Contents}

1 Introduction . . . . . . . . . . . 1

2 Compton scattering in $\mathrm{B} \chi \mathrm{PT} \ldots \ldots . \ldots 2$

2.1 Power counting . . . . . . . . . . . . 2

2.2 Complete NLO calculation in the Delta-region . 3

2.3 Remarks on higher-order Delta contributions . . 4

2.4 Summary ................ . 4

3 Decompositions of the Compton amplitude ... . . 5

4 Static polarisabilities ... . . . . . . . . . 5 5

4.1 Definitions................ . . 5

4.2 Proton . . . . . . . . . . . . 6

4.3 Neutron . . . . . . . . . . . . . . 8

4.4 Error estimates . . . . . . . . . . . . . 9 9

5 Dynamical polarisabilities and multipoles ..... 9

5.1 Definitions . . . . . . . . . . . . . . . 9 9

5.2 Results. . . . . . . . . . . . . . 10

6 Polarised observables for the proton . . . . . . . . 15

7 Conclusion . . . . . . . . . . . . . . 18

Appendix A: Transformation between the bases . . . . 19 Appendix B: Matching of higher-order polarisabilities . 21 Appendix C: Expressions for static polarisabilities . . . 23

$1 \pi \mathrm{N}$ loops . . . . . . . . . . . . . 23

a Proton . . . . . . . . . . . 23

b Neutron . . . . . . . . . . . . . . 24

$2 \pi \Delta$ loops . . . . . . . . . . . . . 2 25

$3 \Delta$ excitation . . . . . . . . . . 27

Appendix D: Details of higher-order Delta self-energy . 27

References . . . . . . . . . . . . . . . . . 28

\section{Introduction}

Low-energy Compton scattering off the nucleon is traditionally used to access the nucleon polarisabilities, but the 
relation between the observables and the polarisabilities is not straightforward; see Refs. [1-3] for recent reviews. A direct relation only exists at the level of the low-energy expansion (LEX), which is a polynomial expansion in the photon energy $\omega$. The LEX validity is limited to very low energies, well below the pion production threshold $\left(\omega \ll m_{\pi}\right)$. Most of the experimental data are, however, obtained at energies above $100 \mathrm{MeV}$. Chiral perturbation theory $(\chi \mathrm{PT})[4,5]$ in the single-nucleon sector [6], as a lowenergy effective-field theory of QCD, can provide the necessary model-independent link between the nucleon Comptonscattering data and polarisabilities beyond the LEX applicability. In recent years $\chi \mathrm{PT}$ calculations have been performed using both the heavy-baryon (HB $\chi \mathrm{PT})$ and manifestly Lorentz-covariant (B $\chi \mathrm{PT}$ ) formulations; see, respectively, [7-11] and [12-14]; some details of the differences between the two approaches can be found in [15]. These calculations are not only useful for precision determination of the polarisabilities from experiment - they also provide a testing ground for $\chi \mathrm{PT}$ in the single nucleon sector. ${ }^{1}$

In this paper we follow up on the manifestly covariant calculation of Ref. [13], where the next-to-next-to-leading order (NNLO) calculation of proton Compton scattering was carried out in $\mathrm{SU}(2) \mathrm{B} \chi \mathrm{PT}$ with pion, nucleon and Delta(1232) degrees of freedom. This NNLO calculation provides a prediction for all the nucleon polarisabilities and scattering observables; however, only the proton scalar dipole polarisabilities and the unpolarised differential cross sections were examined in Ref. [13]. In the present paper we consider a number of the other predictions. We correct the numerical results for the dipole polarisabilities of the proton and present the corresponding results for the neutron. We present the NNLO results, both analytically and numerically, for the quadrupole polarisabilities and the four dipole spin polarisabilities. We also extend the calculation of [13] to the Delta(1232)-resonance region and present results for the Compton multipoles and dynamical polarisabilities. In doing so, we shall remark on a subtlety in the matching of the multipole expansion and static polarisabilities. Finally, we shall consider the B $\chi$ PT predictions for the polarised proton observables, namely the asymmetries $\Sigma_{3}, \Sigma_{2 x}, \Sigma_{2 z}$, and $\Sigma_{1 z}$. These results should be of particular interest to the ongoing Compton-scattering experiments at MAMI (Mainz) and HIGS (Duke) facilities, which aim to determine the scalar and spin polarisabilities of the proton and neutron in polarised measurements [16-18].

The paper is structured as follows. In Sect. 2 we recall the main ingredients of the NNLO B $\chi$ PT calculation of Compton

\footnotetext{
${ }^{1}$ Previous work on the polarisabilities of the nucleons in the framework of $\chi$ PT is contained in Refs. [19-29], and in a chiral framework in [30].
}

scattering. In Sect. 3 we introduce various parametrisations of the Compton amplitude and establish relations between them. We provide the predictions of $\mathrm{B} \chi \mathrm{PT}$ for the nucleon static polarisabilities in Sect. 4, and the Compton multipoles and dynamical polarisabilities in Sect. 5, and compare them with various empirical and theoretical results. Section 6 presents our predictions for some of the polarised observables, compared with experimental data where possible. We conclude with Sect. 7.

\section{Compton scattering in $\mathrm{B} \chi \mathrm{PT}$}

Following Refs. [12-14], where one can find details such as the relevant $\chi \mathrm{PT}$ Lagrangians, we consider low-energy Compton scattering in $\mathrm{B} \chi \mathrm{PT}$, i.e., a manifestly covariant formulation of $\chi \mathrm{PT}$ with pion, nucleon and Delta(1232) isobar degrees of freedom; see Ref. [31, Sec. 4] for a review. Our present calculation of the Compton amplitude follows that of Ref. [13] below photoproduction threshold (and hence for the static polarisabilities), but improves the treatment of the Delta-excitation near the resonance, as described below.

\subsection{Power counting}

Our EFT expansion uses the $\delta$-counting [10], where the mass difference between the nucleon and the Delta, $\Delta=M_{\Delta}-$ $M_{\mathrm{N}}$, is considered an intermediate scale, so that

$m_{\pi} / \Delta \simeq \Delta / \Lambda_{\chi} \equiv \delta$

and hence the usual chiral expansion scale $m_{\pi} / \Lambda_{\chi}$ is counted as $\delta^{2}$. An important feature of the $\delta$-counting is that the characteristic momentum $p$ distinguishes two regimes: low energy: $p \simeq m_{\pi}$, and Delta-resonance: $p \simeq \Delta$.

Since the Delta propagators go as $1 /(p \pm \Delta)$, rather than simply $1 / p$ as for the nucleon, the counting of the graphs with Deltas is different in the two regimes. For example, replacing a nucleon propagator by a Delta changes the power of the graph by $p / \Delta$, which is $O\left(p^{1 / 2}\right)$ in the low-energy regime and $O(1)$ in the resonance region. In addition, the counting demands that the one-Delta-reducible $(1 \Delta \mathrm{R})$ loop graphs, which contain the pole at $p=\Delta$, are resummed in the regime $p \simeq \Delta$, which gives rise to the Delta-resonance width and results in a natural description of the resonance peak.

In general, a graph with $L$ loops, $N_{\pi}$ pion propagators, $N_{N}$ nucleon propagators, $N_{\Delta}$ Delta propagators and $V_{k}$ vertices of order $k$ counts in the low-energy regime as $\mathscr{O}\left(p^{n}\right)$, with

$n=4 L-2 N_{\pi}-N_{N}-\frac{1}{2} N_{\Delta}+k V_{k}$

In the resonance regime, one identifies the number of the $1 \Delta \mathrm{R}$ propagators, $N_{1 \Delta \mathrm{R}}$, then 
$n=4 L-2 N_{\pi}-N_{N}-N_{\Delta}-2 N_{1 \Delta \mathrm{R}}+k V_{k}$.

In the low-energy regime, which is most relevant for the extraction of nucleon polarisabilities, the diagrams with $N_{\Delta}$ Delta propagators are suppressed by $\delta^{N_{\Delta}}$, or equivalently by $p^{N_{\Delta} / 2}$, with respect to the same diagram but with nucleon propagators. The leading order (LO) contribution in this regime hence comes from the nucleon Born term (first two diagrams in Fig. 1) which are responsible, for instance, for the correct Thomson limit. Inclusion of the nucleon anomalous magnetic moment in these graphs is warranted at $\mathscr{O}\left(p^{3}\right)$, which here is the next-to-leading order (NLO).

Also of $\mathscr{O}\left(p^{3}\right)$ is the $\pi^{0}$-pole graph in Fig. 1, and all of the pion-nucleon loops in Fig. 2. These graphs give the LO contribution to the polarisabilities, as the Born graphs do not contribute to polarisabilities by definition. The Deltapole and pion-Delta loop graphs, Fig. 3, enter at $\mathscr{O}\left(p^{7 / 2}\right)$, here the next-to-next-to-leading order (NNLO), and give the NLO contribution to polarisabilities. We note that the Deltapole term, despite being formally suppressed compared to the pion-nucleon loops, has long been known to give a large contribution to the magnetic polarisabilities of the nucleon.

The NNLO amplitude thus receives contributions from the nucleon Born and $\pi^{0}$ pole graphs of Fig. 1, the $\pi \mathrm{N}$ loop graphs of Fig. 2 and the Delta graphs in Fig. 3; all of these were computed in [13].

\subsection{Complete NLO calculation in the Delta-region}

In order to extend this calculation to the Delta-resonance region, we resum the $\pi \mathrm{N}$ loop contributions to the Delta propagator in the Delta-pole graphs, as explained in Ref. [10]. In addition we take into account the leading pion-loop corrections to the $\gamma N \Delta$ vertex, shown in Fig. 4. Those additions
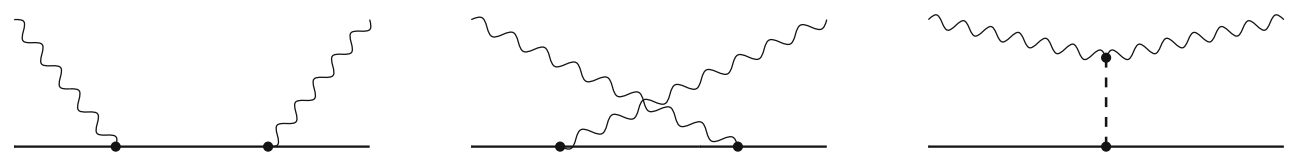

Fig. 1 Born graphs and the anomaly graph. Dots are vertices from the lowest-order Lagrangians
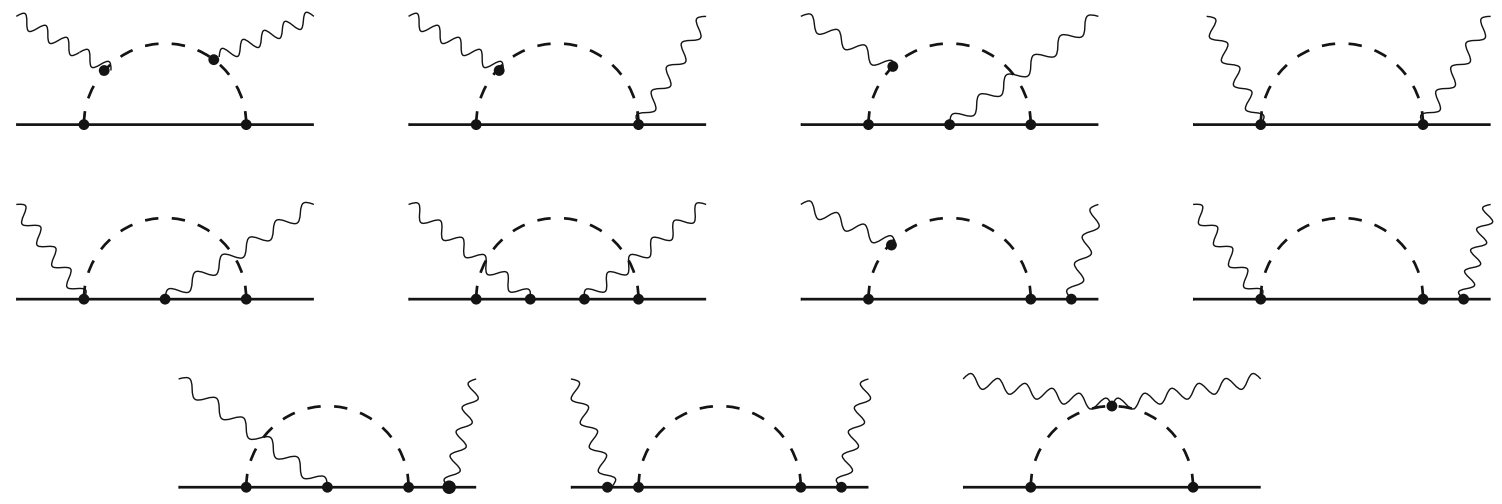

Fig. 2 Pion-nucleon loops that contribute to nucleon polarisabilities at leading order. Crossed and time-reversed graphs are not shown but are included in the calculation
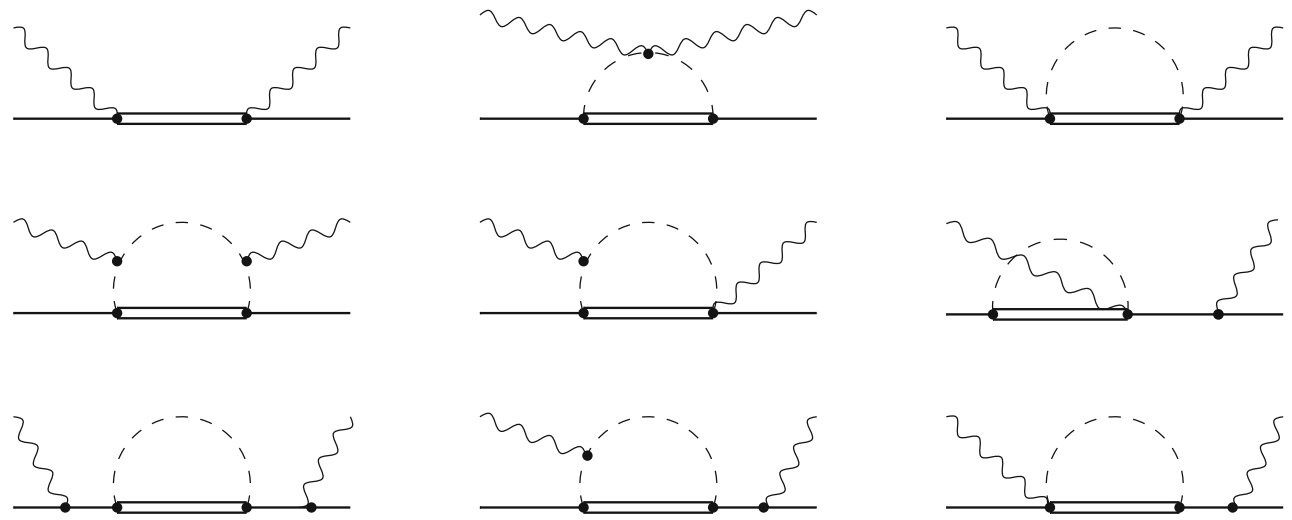

Fig. 3 Pion-Delta loops and the Delta tree graph that contribute to nucleon polarisabilities at next-to-leading order. Crossed and time-reversed graphs are not shown but are included in the calculation. Double lines denote the propagator of the Delta 
are important for unitarity above the pion threshold. At NLO in the regime $p \simeq \Delta$, only the imaginary part of these vertex loops is relevant (specific remarks on the real part are given in Sect. 2.3); this means that these corrections vanish below the pion production threshold and hence do not affect the static polarisabilities. We will, however, investigate their importance for the dynamical polarisabilities and Compton multipoles; see Sect. 5. The technical details of the counting and the evaluation of these $\gamma N \Delta$-vertex corrections are found in Refs. [11,32].

\subsection{Remarks on higher-order Delta contributions}

The effects of running of the $\gamma N \Delta$ coupling constants $g_{M}$ and $g_{E}$, arising due to the real parts of the $\gamma N \Delta$ vertex corrections Fig. 4, are beyond the order we are working at in the Delta region [10]. Nevertheless, we briefly consider the potential impact of these effects, together with the effects of the running of the Delta mass and field renormalisation constants. None of these effects are included in subsequent section; they are also not the only higher-order effects which could impact the polarisabilities. Of the latter, the inclusion of the anomalous magnetic moments of the proton and neutron within $\pi \mathrm{N}$ loop graphs may well be significant [24-27].

The running of the real part of the $\gamma N \Delta$ couplings from the graphs of Fig. 4 was calculated in $[11,32]$. The corresponding numerical results are shown in the left panel of Fig. 5. Both coupling are seen to be reduced, in absolute value, at low energy, and hence the Delta-pole contribution to the polarisabilities will be reduced. On the other hand, this effect is partially compensated by the one-loop running of the Delta propagator, which reads

$S_{\mu \nu}(p)=-\frac{1+\tau_{R}(s)}{\not p-M_{\Delta}(s)+i \Gamma_{\Delta}(s) / 2} \mathcal{P}_{\mu \nu}^{3 / 2}(p)$,
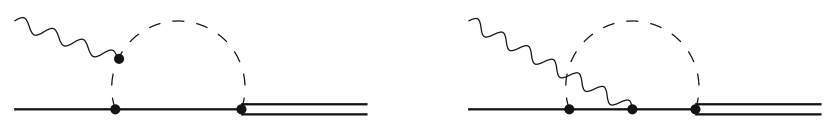

Fig. $4 \gamma N \Delta$ vertex with NLO loop corrections. Only the imaginary parts of the loops shown here lead to contributions at next-to-leading order around the Delta peak where $\mathcal{P}_{\mu \nu}^{3 / 2}(p)$ is the spin-3/2 projection operator, $M_{\Delta}(s)$ is the running Delta mass, and $\Gamma_{\Delta}(s)$ is the width of the Delta. The renormalised self-energy and field renormalisation are calculated as shown in Appendix D.

The resulting $M_{\Delta}(s)$ and $1+\operatorname{Re} \tau_{R}(s)$ are shown Fig. 5 and are seen thus to strengthen the Delta-pole effects at lower energies. A complete $\mathcal{O}\left(p^{4}\right) \mathrm{B} \chi \mathrm{PT}$ calculation will need to be done to correctly quantify the effect of these contributions. This calculation is, however, beyond the scope of the present work.

\subsection{Summary}

With these ingredients, our calculation of the amplitudes is NNLO $\left[\mathscr{O}\left(p^{7 / 2}\right)\right]$ at low energies and NLO $\left[\mathscr{O}\left(p^{2}\right)\right]$ in the Delta resonance region, as summarised in Table 1.

The numerical values of the physical constants used in the present calculation are given in Table 2 . They are only marginally different from the ones used in [13], and the same as in [15].

Table 1 Counting the graphs in Figs. 1, 2, 3 and 4 in the two different regimes

Source $\quad N$ and $\pi^{0}$ pole $\pi \mathrm{N}$ loops $\pi \Delta$ loops $\Delta$ pole $\Delta$-pole corr.

Regime

$$
\begin{array}{llllll}
p \simeq m_{\pi} & p^{2}+p^{3} & p^{3} & p^{7 / 2} & p^{7 / 2} & p^{4} \\
p \simeq \Delta & p^{2}+p^{3} & p^{3} & p^{3} & p & p^{2}
\end{array}
$$

Table 2 Parameters used in our calculation (the $\pi^{0}$ mass is only used for the computation of the $t$-channel pion-pole graph). Most of the values are from Particle Data Group [33]. The $\pi N \Delta$ coupling constant $h_{A}$ is fit to the experimental Delta width and the magnetic and electric $\gamma N \Delta$ coupling constants $g_{M}$ and $g_{E}$ are taken from the pion photoproduction study of Ref. [32]. More details can be found in Ref. [11]

\begin{tabular}{lll}
\hline$\alpha_{\mathrm{em}}=1 / 137.04$ & $g_{A}=1.270$ & $f_{\pi}=92.21 \mathrm{MeV}$ \\
$m_{\pi^{ \pm}}=139.57 \mathrm{MeV}$ & $m_{\pi^{0}}=134.98 \mathrm{MeV}$ & \\
$M_{\mathrm{N}} \equiv M_{p}=938.27 \mathrm{MeV}$ & $\kappa_{p}=1.793$ & $M_{\Delta}=1232 \mathrm{MeV}$ \\
$h_{A} \equiv 2 g_{\pi \mathrm{N} \Delta}=2.85$ & $g_{M}=2.97$ & $g_{E}=-1.0$ \\
\hline
\end{tabular}
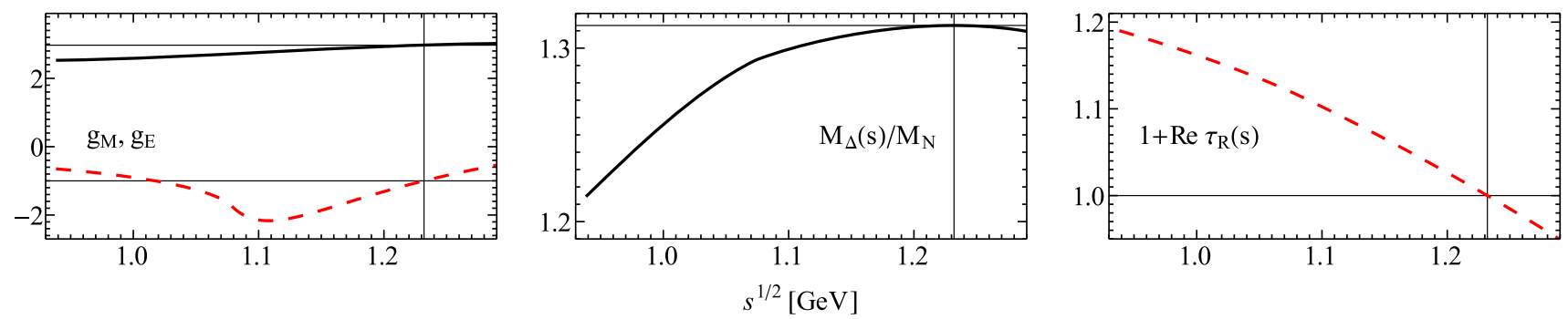

Fig. 5 Running of the $\gamma N \Delta$ coupling constants $g_{M}$ and $g_{E}$ (left panel, solid/dashed line, respectively), of the Delta mass (centre panel), and of the field renormalisation constant (right panel), as functions of $\sqrt{s}$ in GeV. Grid lines represent the values renormalised at the Delta peak and the corresponding value of $\sqrt{s}$ 


\section{Decompositions of the Compton amplitude}

Before presenting the results for the proton polarisabilities and Compton observables, we provide details of the tensor decomposition of the reaction amplitude. To compute the Compton-scattering amplitude for a spin- $1 / 2$ target in a manifestly Lorentz and gauge invariant form, we use a set of eight covariant tensors [10]:

$T_{f i}=\mathcal{E}_{\mu}^{*}\left(q^{\prime}\right) \mathcal{E}_{v}(q) \sum_{i=1}^{8} \mathcal{A}_{i}(s, t) \bar{u}_{s^{\prime}}\left(p^{\prime}\right) O_{i}^{\mu v} u_{s}(p)$,

where $\mathcal{A}_{1} \ldots \mathcal{A}_{8}$ are the invariant scalar amplitudes with the Mandelstam variables $s, t, u$ defined as usual. The final and initial 4-momenta of the nucleon (and photon) are denoted as $p^{\prime}, p$ (and $q^{\prime}, q$ ), respectively, and obey the on-mass-shell conditions: $p^{\prime 2}=p^{2}=M_{\mathrm{N}}^{2}, q^{\prime 2}=q^{2}=0$. The free nucleon spinor $u_{s}(p)$ is normalised to $2 M_{\mathrm{N}}$, whereas $\mathcal{E}_{\mu}$ is a modified photon polarisation vector:

$\mathcal{E}_{\mu}(q)=\varepsilon_{\mu}-\frac{P \cdot \varepsilon}{P \cdot q} q_{\mu}$,

with $P=p+p^{\prime}$. The tensors $O_{i}$ are given by

$O_{1}^{\mu \nu}=-g^{\mu \nu}$,

$O_{2}^{\mu \nu}=q^{\mu} q^{\prime \nu}$,

$O_{3}^{\mu \nu}=-\gamma^{\mu \nu}$,

$O_{4}^{\mu \nu}=g^{\mu \nu}\left(q^{\prime} \cdot \gamma \cdot q\right)$,

$O_{5}^{\mu \nu}=q^{\mu} q_{\alpha}^{\prime} \gamma^{\alpha \nu}+\gamma^{\mu \alpha} q_{\alpha} q^{\prime \nu}$,

$O_{6}^{\mu \nu}=q^{\mu} q_{\alpha} \gamma^{\alpha \nu}+\gamma^{\mu \alpha} q_{\alpha}^{\prime} q^{\prime \nu}$,

$O_{7}^{\mu \nu}=q^{\mu} q^{\prime \nu}\left(q^{\prime} \cdot \gamma \cdot q\right)$,

$O_{8}^{\mu \nu}=\gamma^{\mu \nu \alpha \beta} q_{\alpha} q_{\beta}^{\prime}=-i \gamma_{5} \epsilon^{\mu \nu \alpha \beta} q_{\alpha}^{\prime} q_{\beta}$,

where $\gamma^{\mu \nu}=\frac{1}{2}\left[\gamma^{\mu}, \gamma^{\nu}\right], \gamma^{\mu \nu \alpha \beta}=\frac{1}{2}\left[\gamma^{\mu \nu \alpha}, \gamma^{\beta}\right]$ with $\gamma^{\mu \nu \alpha}=\frac{1}{2}\left\{\gamma^{\mu \nu}, \gamma^{\alpha}\right\}$, and $\epsilon^{0123}=-1$. The representation (5) is obtained by writing down the most general covariant structure (for the on-shell case) and imposing the electromagnetic current-conservation condition. The amplitudes $A_{i}$ are most easily computed in the following Lorenz-invariant gauge:

$P \cdot \varepsilon=0=P \cdot \varepsilon^{\prime}$.

This condition can also be achieved in the Coulomb gauge $\left(\varepsilon_{0}=0=\varepsilon_{0}^{\prime}\right.$ ) by going to the Breit frame: $\vec{P}=0$.

The set of tensors $O_{i}$ is overcomplete. Alternative covariant bases containing only six basis tensors have been used in the literature, in particular the Hearn-Leader basis, a slight variant of which is used in Ref. [34] with corresponding amplitudes denoted $T_{i}$. From these though a more convenient set of amplitudes can be constructed, which we will refer to as the L'vov amplitudes $A_{i}^{\mathrm{L}}$ (they are simply $A_{i}$ in [34]). These crossing-symmetric amplitudes are routinely used in dispersion-relation calculations of Compton amplitudes and are a convenient bridge between other sets of amplitudes, cf. Appendix A.

In addition, by representing the nucleon Dirac spinors via their upper and lower Pauli spinors, the tensors $O_{i}$ can be decomposed into a set of non-relativistic basis tensors $t_{i}$. In the centre-of-mass (c.m.) or Breit frames (in both of which the incoming and outgoing photon energies are equal) there are six such tensors, and one obtains the following decomposition of the amplitude [22]:

$$
\begin{aligned}
T_{f i}= & 2 M_{\mathrm{N}} \sum_{i=1}^{6} A_{i}(\omega, \theta) \chi_{s^{\prime}}^{\dagger} t_{i} \chi_{s} \\
= & 2 M_{\mathrm{N}} \chi_{s^{\prime}}^{\dagger}\left(A_{1}(\omega, \theta) \vec{\epsilon}^{*} \cdot \vec{\epsilon}+A_{2}(\omega, \theta) \vec{\epsilon}^{*} \cdot \hat{q} \vec{\epsilon} \cdot \hat{q}^{\prime}\right. \\
& +i A_{3}(\omega, \theta) \vec{\sigma} \cdot\left(\vec{\epsilon}^{\prime} \times \vec{\epsilon}\right)+i A_{4}(\omega, \theta) \vec{\sigma} \cdot\left(\hat{q}^{\prime} \times \hat{q}\right) \vec{\epsilon}^{*} \cdot \vec{\epsilon} \\
& +i A_{5}(\omega, \theta) \vec{\sigma} \cdot\left[\left(\vec{\epsilon}^{\prime *} \times \hat{q}\right) \vec{\epsilon} \cdot \hat{q}^{\prime}-\left(\vec{\epsilon} \times \hat{q}^{\prime}\right) \vec{\epsilon}^{\prime *} \cdot \hat{q}\right] \\
& \left.+i A_{6}(\omega, \theta) \vec{\sigma} \cdot\left[\left(\vec{\epsilon}^{*} \times \hat{q}^{\prime}\right) \vec{\epsilon} \cdot \hat{q}^{\prime}-(\vec{\epsilon} \times \hat{q}) \vec{\epsilon}^{*} \cdot \hat{q}\right]\right) \chi_{s},
\end{aligned}
$$

where now $\vec{\epsilon}^{\prime}(\vec{\epsilon})$ are the final (initial) photon polarisation vectors, $\hat{q}^{\prime}(\hat{q})$ are the final (initial) photon momentum unit vectors, $\omega$ and $\theta$ are the photon energy and scattering angle, $\vec{\sigma}$ the Pauli matrices, and $\chi_{s}$ are Pauli spinors. The $A_{i}$ are of course frame-dependent, and we will denote them $A_{i}^{\mathrm{cm}}$ and $A_{i}^{\mathrm{Br}}$ for the c.m. and Breit frames, respectively.

In the present calculation, our starting point is the eight Compton amplitudes $\mathcal{A}_{i}(s, t)$, as obtained in Ref. [13] with the modifications described in Sect. 2. These can be transformed into the any of the minimal sets $A_{i}$ as follows:

$A_{i}(\omega, \theta)=\sum_{j=1}^{8} C_{i j}^{\mathcal{A} \rightarrow A}(s, t) \mathcal{A}_{j}(s, t)$

the explicit expression for the $6 \times 8$ matrix $C^{\mathcal{A} \rightarrow A}(s, t)$ for various sets of amplitudes are discussed in Appendix A. For the study of Compton multipoles and dynamical nucleon polarisabilities the c.m. frame amplitudes are the natural basis, while the connection to static polarisabilities is more easily made via the L'vov or Breit amplitudes.

\section{Static polarisabilities}

\subsection{Definitions}

The polarisabilities characterise the quadratic response of the external electric and magnetic fields, $\vec{E}$ and $\vec{H}$. In moderate 
fields the response of the nucleon can be described by the following effective Hamiltonians [23,34]: ${ }^{2}$

$$
\begin{aligned}
\mathcal{H}_{\mathrm{eff}}^{(2)}= & -\frac{1}{2} 4 \pi\left(\alpha_{E 1} \vec{E}^{2}+\beta_{M 1} \vec{H}^{2}\right), \\
\mathcal{H}_{\mathrm{eff}}^{(3)}= & -\frac{1}{2} 4 \pi\left(\gamma_{E 1 E 1} \vec{\sigma} \cdot \vec{E} \times \dot{\vec{E}}+\gamma_{M 1 M 1} \vec{\sigma} \cdot \vec{H} \times \dot{\vec{H}}\right. \\
& \left.-2 \gamma_{M 1 E 2} E_{i j} \sigma_{i} H_{j}+2 \gamma_{E 1 M 2} H_{i j} \sigma_{i} E_{j}\right) \\
\mathcal{H}_{\mathrm{eff}}^{(4)}= & -\frac{1}{2} 4 \pi\left(\alpha_{E 1 \nu} \dot{\overrightarrow{\vec{E}}}^{2}+\beta_{M 1 \nu} \dot{\vec{H}}^{2}\right) \\
& -\frac{1}{12} 4 \pi\left(\alpha_{E 2} E_{i j}^{2}+\beta_{M 2} H_{i j}^{2}\right), \\
\mathcal{H}_{\mathrm{eff}}^{(5)}= & -\frac{1}{2} 4 \pi\left(\gamma_{E 1 E 1 \nu} \vec{\sigma} \cdot \dot{\vec{E}} \times \ddot{\vec{E}}+\gamma_{M 1 M 1 \nu} \vec{\sigma} \cdot \dot{\vec{H}} \times \ddot{\vec{H}}\right. \\
& -2 \gamma_{M 1 E 2 \nu} \sigma_{i} \dot{E}_{i j} \dot{H}_{j}+2 \gamma_{E 1 M 2 \nu} \sigma_{i} \dot{H}_{i j} \dot{E_{j}} \\
& +4 \gamma_{E 2 E 2} \epsilon_{i j k} \sigma_{i} E_{j \ell} \dot{E}_{k \ell}+4 \gamma_{M 2 M 2} \epsilon_{i j k} \sigma_{i} H_{j \ell} \dot{H}_{k \ell} \\
& \left.-6 \gamma_{M 2 E 3} \sigma_{i} E_{i j k} H_{j k}+6 \gamma_{E 2 M 3} \sigma_{i} H_{i j k} E_{j k}\right),
\end{aligned}
$$

where

$$
\begin{aligned}
E_{i j}= & \frac{1}{2}\left(\nabla_{i} E_{j}+\nabla_{j} E_{i}\right), \\
E_{i j k}= & \frac{1}{3}\left(\nabla_{i} \nabla_{j} E_{k}+\nabla_{i} \nabla_{k} E_{j}+\nabla_{j} \nabla_{k} E_{i}\right) \\
& -\frac{1}{15}\left(\delta_{i j} \nabla^{2} E_{k}+\delta_{j k} \nabla^{2} E_{i}+\delta_{i k} \nabla^{2} E_{j}\right),
\end{aligned}
$$

and similarly for $H$.

The non-relativistic Hamiltonian is defined only in a specific reference frame. As far as the Compton-scattering process is concerned, the Breit frame yields the most natural description because of the manifest crossing symmetry of the corresponding amplitudes. The contribution of $\mathcal{H}_{\text {eff }}$ to the Breit-frame amplitudes reads

$$
\begin{aligned}
A_{1}^{\mathrm{Br}}= & +\frac{4 \pi E_{\mathrm{N}}}{M_{\mathrm{N}}}\left(\left(\alpha_{E 1}+z \beta_{M 1}\right) \omega_{B}^{2}\right. \\
& \left.+\left(\alpha_{E 1 v}+z \beta_{M 1 v}-\frac{1}{12} \beta_{M 2}+z \frac{1}{12} \alpha_{E 2}+\frac{1}{6} z^{2} \beta_{M 2}\right) \omega_{B}^{4}\right), \\
A_{2}^{\mathrm{Br}}= & +\frac{4 \pi E_{\mathrm{N}}}{M_{\mathrm{N}}}\left(-\beta_{M 1} \omega_{B}^{2}+\left(-\beta_{M 1 v}+\frac{1}{12} \alpha_{E 2}-\frac{1}{6} z \beta_{M 2}\right) \omega_{B}^{4}\right), \\
A_{3}^{\mathrm{Br}}= & -\frac{4 \pi E_{\mathrm{N}}}{M_{\mathrm{N}}}\left(\left(\gamma_{E 1 E 1}+\gamma_{E 1 M 2}+z\left(\gamma_{M 1 M 1}+\gamma_{M 1 E 2}\right)\right) \omega_{B}^{3}\right. \\
& +\left(\gamma_{E 1 E 1 v}+\gamma_{E 1 M 2 v}+z\left(\gamma_{M 1 M 1 v}+\gamma_{M 1 E 2 v}\right)\right. \\
& -\frac{12}{5} \gamma_{M 2 E 3}-3 \gamma_{M 2 M 2}+z\left(\gamma_{E 2 E 2}+\frac{8}{5} \gamma_{E 2 M 3}\right) \\
& \left.\left.+4 z^{2}\left(\gamma_{M 2 M 2}+\gamma_{M 2 E 3}\right)\right) \omega_{B}^{5}\right),
\end{aligned}
$$

\footnotetext{
2 The notation differs slightly; for instance Ref. [23] used $\gamma_{E 2}$ for $\gamma_{M 1 E 2}$ and $\gamma_{E T}$ for $\gamma_{E 2 E 2 \text {. }}$
}

$$
\begin{aligned}
A_{4}^{\mathrm{Br}}= & +\frac{4 \pi E_{\mathrm{N}}}{M_{\mathrm{N}}}\left(\left(\gamma_{M 1 E 2}-\gamma_{M 1 M 1}\right) \omega_{B}^{3}+\left(\gamma_{M 1 E 2 v}-\gamma_{M 1 M 1 v}\right.\right. \\
& \left.\left.-\gamma_{E 2 E 2}-\frac{2}{5} \gamma_{E 2 M 3}+z\left(2 \gamma_{M 2 E 3}-4 \gamma_{M 2 M 2}\right)\right) \omega_{B}^{5}\right) \\
A_{5}^{\mathrm{Br}}= & +\frac{4 \pi E_{\mathrm{N}}}{M_{\mathrm{N}}}\left(\gamma_{M 1 M 1} \omega_{B}^{3}+\left(\gamma_{M 1 M 1 v}-\gamma_{E 2 E 2}\right.\right. \\
& \left.\left.-\gamma_{E 2 M 3}+z\left(4 \gamma_{M 2 M 2}+\gamma_{M 2 E 3}\right)\right) \omega_{B}^{5}\right) \\
A_{6}^{\mathrm{Br}}= & +\frac{4 \pi E_{\mathrm{N}}}{M_{\mathrm{N}}}\left(\gamma_{E 1 M 2} \omega_{B}^{3}+\left(\gamma_{E 1 M 2 v}-\frac{7}{5} \gamma_{M 2 E 3}\right.\right. \\
& \left.\left.-2 \gamma_{M 2 M 2}+3 z \gamma_{E 2 M 3}\right) \omega_{B}^{5}\right)
\end{aligned}
$$

where $\omega_{B}$ and $z=\cos \theta_{B}$ refer here to the Breit-frame photon energy and scattering angle, and $E_{\mathrm{N}}$ is the nucleon energy. This is a complete low-energy expansion of the non-Born part of the Compton amplitudes to order $\omega_{B}^{5}$.

In addition to the above polarisabilities, we shall examine the forward and backward spin polarisabilities $\gamma_{0}$ and $\gamma_{\pi}$ defined as

$$
\begin{aligned}
& \gamma_{0}=-\gamma_{E 1 E 1}-\gamma_{M 1 M 1}-\gamma_{E 1 M 2}-\gamma_{M 1 E 2}, \\
& \gamma_{\pi}=\gamma_{M 1 M 1}+\gamma_{M 1 E 2}-\gamma_{E 1 E 1}-\gamma_{E 1 M 2},
\end{aligned}
$$

and the so-called "higher-order forward spin polarisability" $\bar{\gamma}_{0}$, defined as a linear combination of the quadrupole spin polarisabilities [35]:

$$
\begin{aligned}
\bar{\gamma}_{0}= & -\gamma_{E 1 E 1 v}-\gamma_{M 1 M 1 v}-\gamma_{E 1 M 2 v}-\gamma_{M 1 E 2 v} \\
& -\gamma_{E 2 E 2}-\gamma_{M 2 M 2}-\frac{8}{5}\left(\gamma_{E 2 M 3}+\gamma_{M 2 E 3}\right) .
\end{aligned}
$$

The covariant $\chi \mathrm{PT}$ expressions for the polarisabilities are given in Appendix C, with the corresponding numerical values given below, for the proton and neutron separately. These expressions were obtained by computing the covariant amplitudes $\mathcal{A}_{i}$, converting them to the Breit-frame amplitudes as discussed above, expanding in $\omega_{B}$ and $z$ and identifying the coefficients with the polarisabilities. Alternatively, one can go via the L'vov amplitudes, as shown in Appendix B where we give their complete and consistent relation to the static polarisabilities of Eqs. (11-14). For example, $\bar{\gamma}_{0}=a_{4, v} / 2 \pi M_{\mathrm{N}}$, where $a_{4, v}$ is a coefficient of the Taylor expansion of $A_{4}^{\mathrm{L}}$, Eq. (B1).

\subsection{Proton}

Our results for the scalar dipole and quadrupole and spin dipole static polarisabilities are shown in Tables 3 and 4. The analytical expressions for the $\pi \mathrm{N}$ loop, $\pi \Delta$ loop, and Delta-pole contributions to these polarisabilities are given in Appendix C. We also show results obtained by other authors. The latter are a mixture of predictions and fits to Compton- 
Table 3 Values of proton static dipole, quadrupole, and dispersive polarisabilities, in units of $10^{-4} \mathrm{fm}^{3}$ (dipole) and $10^{-4} \mathrm{fm}^{5}$ (quadrupole and dispersive), in comparison with the fixed- $t$ DR extraction of
Refs. [23, 34,38] and $\chi$ PT fits. The results of the calculation of Ref. [38] are kindly provided by Pasquini [39]

\begin{tabular}{|c|c|c|c|c|c|c|}
\hline Source & $\alpha_{E 1}$ & $\beta_{M 1}$ & $\alpha_{E 2}$ & $\beta_{M 2}$ & $\alpha_{E 1 v}$ & $\beta_{M 1 v}$ \\
\hline $\mathscr{O}\left(p^{3}\right) \pi \mathrm{N}$ loops & 6.9 & -1.8 & 13.5 & -8.4 & 0.7 & 1.8 \\
\hline $\mathscr{O}\left(p^{7 / 2}\right) \pi \Delta$ loops & 4.4 & -1.4 & 3.2 & -2.7 & -0.6 & 0.6 \\
\hline$\Delta$ pole & -0.1 & 7.1 & 0.6 & -4.5 & -1.5 & 4.7 \\
\hline Total & $11.2 \pm 0.7$ & $3.9 \pm 0.7$ & $17.3 \pm 3.9$ & $-15.5 \pm 3.5$ & $-1.3 \pm 1.0$ & $7.1 \pm 2.5$ \\
\hline Fixed- $t$ DR $[34,37]$ & $12.1^{\mathrm{a}}$ & $1.6^{\mathrm{a}}$ & 27.5 & -22.4 & -3.8 & 9.1 \\
\hline Fixed- $t$ DR $[23,38]$ & $\cdots$ & $\cdots$ & 27.7 & -24.4 & -3.9 & 9.3 \\
\hline $\mathrm{HB} \chi \mathrm{PT}$ fit [11] & $10.65 \pm 0.50$ & $3.15 \pm 0.50$ & $\cdots$ & $\cdots$ & $\cdots$ & $\cdots$ \\
\hline $\mathrm{B} \chi \mathrm{PT}$ fit $[14]$ & $10.6 \pm 0.5$ & $3.2 \pm 0.5$ & $\cdots$ & $\cdots$ & $\cdots$ & $\cdots$ \\
\hline PDG [33] & $11.2 \pm 0.4$ & $2.5 \pm 0.4$ & $\cdots$ & $\cdots$ & $\cdots$ & $\cdots$ \\
\hline
\end{tabular}

${ }^{\text {a }}$ Only $\alpha_{E 1}+\beta_{M 1}$ is predicted in DR; the difference is taken from fits to Compton-scattering data

Table 4 Values of proton static spin polarisabilities, in units of $10^{-4} \mathrm{fm}^{4}$ (except for $\bar{\gamma}_{0}$, which is in units of $10^{-4} \mathrm{fm}^{6}$ ), in comparison with the fixed- $t$ DR extraction of Refs. [23,34,38,41] and $\chi \mathrm{PT}$ fits. Reference [18] is an extraction from asymmetry data in the DR framework of Ref. [43], with $\gamma_{0}$ and $\gamma_{\pi}$ as input. Note that the up-todate fixed- $t$ DR values of the proton spin polarisabilities corresponding to Refs. $[23,38,41]$ are also taken from Ref. [18]. The fixed- $t$ DR value of $\bar{\gamma}_{0}$ is from Ref. [35]

\begin{tabular}{|c|c|c|c|c|c|c|c|}
\hline Source & $\gamma_{E 1 E 1}$ & $\gamma_{M 1 M 1}$ & $\gamma_{E 1 M 2}$ & $\gamma_{M 1 E 2}$ & $\gamma_{0}$ & $\gamma_{\pi}$ & $\bar{\gamma}_{0}$ \\
\hline $\mathscr{O}\left(p^{3}\right) \pi \mathrm{N}$ loops & -3.4 & -0.1 & 0.5 & 0.9 & 2.0 & 3.6 & 2.1 \\
\hline $\mathscr{O}\left(p^{7 / 2}\right) \pi \Delta$ loops & 0.4 & -0.2 & 0.1 & -0.2 & -0.1 & -0.9 & -0.01 \\
\hline$\Delta$ pole & -0.4 & 3.3 & -0.4 & 0.4 & -2.8 & 4.4 & -1.0 \\
\hline Total & $-3.3 \pm 0.8$ & $2.9 \pm 1.5$ & $0.2 \pm 0.2$ & $1.1 \pm 0.3$ & $-0.9 \pm 1.4$ & $7.2 \pm 1.7$ & $1.1 \pm 0.5$ \\
\hline Fixed- $t$ DR [34] & -3.4 & 2.7 & 0.3 & 1.9 & -1.5 & 7.8 & $\ldots$ \\
\hline Fixed- $t$ DR $[23,38,41]$ & -4.3 & 2.9 & -0.02 & 2.2 & -0.8 & 9.4 & 0.6 \\
\hline $\mathrm{HB} \chi \mathrm{PT}[11,42]$ & $-1.1 \pm 1.9$ & $2.2 \pm 0.8$ & $-0.4 \pm 0.6$ & $1.9 \pm 0.5$ & $-2.6 \pm 1.9$ & $5.6 \pm 1.9$ & $\cdots$ \\
\hline MAMI 2015 [18] & $-3.5 \pm 1.2$ & $3.16 \pm 0.85$ & $-0.7 \pm 1.2$ & $1.99 \pm 0.29$ & $-1.01 \pm 0.13$ & $8.0 \pm 1.8$ & $\ldots$ \\
\hline
\end{tabular}

scattering data. In most cases the rather well-established constraints obtained via the optical theorem from photoproduction cross sections via the Baldin sum rule for $\alpha_{E 1}+\beta_{M 1}$ [36] have been imposed, and the GDH-like sum rule for $\gamma_{0}$ is also sometimes used.

Table 3 shows the values of the dipole, quadrupole, and dispersive scalar polarisabilities obtained in our calculation, compared with the results of the DR calculations of Refs. [34,38], as well as with the results of the $\chi \mathrm{PT}$ fits $[11,14]$ (in these calculations, only the dipole polarisabilities were fit to the data). The sum of the dipole scalar polarisabilities $\alpha_{E 1}+\beta_{M 1}$ is well constrained by the Baldin sum rule to be $14.0 \pm 0.2$ [40], and even though our central value of 15.1 is somewhat higher than that, they are not in contradiction given the uncertainty of our result.

The fixed- $t$ DR calculations encounter difficulties in describing backward scattering (large $t$ ). As a result they seem to disagree with $\chi \mathrm{PT}$ in the value of $\alpha_{E 1}-\beta_{M 1}$. Fits to experimental data in a DR framework have given results around 10.0-10.5 [34,37], substantially higher than our value of 7.3. The chiral fits give results closer to $7.5[11,14]$, in much better agreement with our prediction.

The values of $\alpha_{E 2}$ and $\beta_{M 2}$ resulting from our calculation are significantly below the corresponding DR values. The same situation is observed in the values of $\alpha_{E 1 v}$ and $\beta_{M 1 v}$; the former is about a factor of two below the DR value.

We note that these significant differences in quadrupole polarisabilities do not show up, in any dramatic fashion, in the Compton observables; both DR and $\chi \mathrm{PT}$ calculations provide a good description of the available data. It could be that the differences in polarisabilities are negated in observables. This is certainly the case for forward scattering, where both calculations agree on the Baldin sum rule, as well as on the (fourth-order) sum rule involving the higher scalar polarisabilities [40].

Table 4 displays the values of the spin dipole polarisabilities predicted in our calculation, compared with the DR results of Refs. $[23,34,38,41]$, and the results of the $\chi \mathrm{PT}$ fit [11]. Here, our results for all the leading spin dipole polarisabilities agree well with the DR ones. The only somewhat discrepant polarisability is $\gamma_{M 1 E 2}$, which is smaller by a fac- 
Table 5 Values of neutron static dipole, quadrupole, and dispersive polarisabilities, in units of $10^{-4} \mathrm{fm}^{3}$ (dipole) and $10^{-4} \mathrm{fm}^{5}$ (quadrupole and dispersive), in comparison with the fixed- $t$ DR extraction of
Refs. $[34,38]$ and $\chi$ PT fits. The results of the calculation of Ref. [38] are kindly provided by Pasquini [39]

\begin{tabular}{|c|c|c|c|c|c|c|}
\hline Source & $\alpha_{E 1}$ & $\beta_{M 1}$ & $\alpha_{E 2}$ & $\beta_{M 2}$ & $\alpha_{E 1 v}$ & $\beta_{M 1 v}$ \\
\hline $\mathscr{O}\left(p^{3}\right) \pi \mathrm{N}$ loops & 9.4 & -1.1 & 12.4 & -8.7 & 2.1 & 1.8 \\
\hline $\mathscr{O}\left(p^{7 / 2}\right) \pi \Delta$ loops & 4.4 & -1.4 & 3.2 & -2.7 & -0.6 & 0.6 \\
\hline$\Delta$ pole & -0.1 & 7.1 & 0.6 & -4.5 & -1.5 & 4.7 \\
\hline Total & $13.7 \pm 3.1$ & $4.6 \pm 2.7$ & $16.2 \pm 3.7$ & $-15.8 \pm 3.6$ & $0.1 \pm 1.0$ & $7.2 \pm 2.5$ \\
\hline Fixed- $t$ DR $[34,44]$ & $12.5^{\mathrm{a}}$ & $2.7^{\mathrm{a}}$ & 27.2 & -23.5 & -2.4 & 9.2 \\
\hline Fixed- $t$ DR $[38,39,43]$ & $\ldots$ & $\ldots$ & 27.9 & -24.3 & -2.8 & 9.3 \\
\hline $\mathrm{HB} \chi \mathrm{PT}$ fit $[1,45]$ & $11.55 \pm 1.5$ & $3.65 \pm 1.5$ & $\ldots$ & $\ldots$ & $\ldots$ & $\ldots$ \\
\hline PDG [33] & $11.6 \pm 1.5$ & $3.7 \pm 2.0$ & $\ldots$ & $\ldots$ & $\cdots$ & $\ldots$ \\
\hline
\end{tabular}

${ }^{\text {a }}$ Only $\alpha_{E 1}+\beta_{M 1}$ is predicted in DR

Table 6 Values of neutron static spin polarisabilities, in units of $10^{-4} \mathrm{fm}^{4}$ (except for $\bar{\gamma}_{0}$, which is in units of $10^{-4} \mathrm{fm}^{6}$ ), in comparison with the fixed- $t$ DR extraction of Refs. [34,38]. The results of the calculation of Ref. [38] are kindly provided by Pasquini [39]

\begin{tabular}{llllllll}
\hline Source & $\gamma_{E 1 E 1}$ & $\gamma_{M 1 M 1}$ & $\gamma_{E 1 M 2}$ & $\gamma_{M 1 E 2}$ & $\gamma_{0}$ & $\bar{\gamma}_{\pi}$ \\
\hline $\mathscr{O}\left(p^{3}\right) \pi \mathrm{N}$ loops & -4.7 & -0.2 & 0.6 & 1.3 & 3.0 & 5.3 & 2.9 \\
$\mathscr{O}\left(p^{7 / 2}\right) \pi \Delta$ loops & 0.4 & -0.2 & 0.1 & -0.2 & -0.1 & -0.9 & -0.01 \\
$\Delta$ pole & -0.4 & 3.3 & -0.4 & 0.4 & -2.8 & 4.5 & -1.0 \\
Total & $-4.7 \pm 1.1$ & $2.9 \pm 1.5$ & $0.2 \pm 0.2$ & $1.6 \pm 0.4$ & $0.03 \pm 1.4$ & $9.0 \pm 2.0$ & $1.9 \pm 0.7$ \\
\hline Fixed- $t$ DR [34] & -5.6 & 3.8 & -0.7 & 2.9 & -0.4 & 13.0 & $\ldots$ \\
Fixed- $t$ DR [38,39,43] & -5.9 & 3.8 & -0.9 & 3.1 & -0.1 & 13.7 \\
HB $\chi$ PT [11,42] & $-4.0 \pm 1.9$ & $1.3 \pm 0.8$ & $-0.1 \pm 0.6$ & $2.4 \pm 0.5$ & $0.5 \pm 1.9$ & $7.7 \pm 1.9$ & $\ldots$ \\
\hline
\end{tabular}

tor of 2 in our calculation. This, in turn, results in our value of $\gamma_{\pi}$ being somewhat smaller. At the same time, our value of $\gamma_{0}$ is very close to that given by the sum rule. ${ }^{3}$ The $\mathrm{HB} \chi \mathrm{PT}$ fit of Ref. [11], on the other hand, gives the values of the spin polarisabilities that also are close to the DR values except for $\gamma_{E 1 E 1}$. Thus, the central values of some of the spin polarisabilities are predicted to be different in $\mathrm{B} \chi \mathrm{PT}$ and in $\mathrm{HB} \chi \mathrm{PT}$ - this can be seen, for instance, in $\gamma_{E 1 E 1}$ or in the forward spin polarisability $\gamma_{0}-$ though it should be noted that they do all agree within the (substantial) combined errors. It is interesting to note that the $\chi \mathrm{PT}$ fits to the unpolarised data done in the two frameworks $[11,14]$, where only $\alpha_{E 1}-\beta_{M 1}$ was fit, resulted in almost identical values of the scalar dipole polarisabilities (cf. Table 3). This demonstrates that the unpolarised low-energy data are not sensitive to the values of the spin polarisabilities (at least to the extent these differ between the $\mathrm{B}$ and the $\mathrm{HB}$ calculations on which the fits were based).

\subsection{Neutron}

In Tables 5 and 6 we, in turn, show our results for the neutron scalar and spin polarisabilities, with the analytical expres-

\footnotetext{
3 We have checked that our value of $\gamma_{0}$ differs from that of Ref. [29] only because the input parameters, particularly $g_{M}$, are slightly different.
}

sions for the $\pi \mathrm{N}$ loop contributions to these polarisabilities given in Appendix C. The $\pi \Delta$ loop and Delta-pole contributions to the neutron polarisabilities are equal at this order to the corresponding proton values.

Table 5 shows the scalar polarisabilities of the neutron, compared with the DR results of Refs. [34,38,39,43] and $\chi$ PT fits of Refs. $[1,45]$. The Baldin sum rule result for the neutron is $\alpha_{E 1}+\beta_{M 1}=15.2 \pm 0.4$ [46], which is again somewhat lower than our central value. Even though our error estimates of the neutron dipole scalar polarisabilities are rather large, one can see that, analogously to the proton, our prediction for $\beta_{M 1}$ is higher than the DR values and closer to those of the chiral fits. The situation with the neutron quadrupole and the dispersive polarisabilities is very similar to the proton case, with $\mathrm{B} \chi \mathrm{PT}$ predictions being significantly smaller than the DR results.

Table 6 shows the neutron spin polarisabilities, compared with the results of the $\mathrm{HB} \chi \mathrm{PT}$ fit [11], and of dispersive evaluations [34,38,39,43] (compatible dispersive evaluations of $\gamma_{0}$ and $\gamma_{\pi}$ for the proton and neutron may also be found in Ref. [47]). Our results for the two largest neutron spin polarisabilities, $\gamma_{E 1 E 1}$ and $\gamma_{M 1 M 1}$, agree well with the DR ones, whereas the magnitudes of $\gamma_{E 1 M 2}$ and $\gamma_{M 1 E 2}$ are predicted by our calculation to be smaller than is obtained in the dispersive calculations, even with the uncertainties taken 
into account. This, in turn, leads in our value of $\gamma_{\pi}$ being somewhat smaller than in the DR framework, with $\gamma_{0}$ being consistent between all frameworks (although not very well constrained). It is interesting that the $\mathrm{HB} \chi \mathrm{PT}$ results follow here a pattern different from those seen in the proton case: they tend to yield a smaller $\gamma_{M 1 M 1}$, rather than $\gamma_{E 1 E 1}$, cf. Table 4 for the proton.

\subsection{Error estimates}

The uncertainties for the total values of static polarisabilities calculated in our work (see the fourth row in Tables 3 , $4,5,6)$ are estimates of the contributions to the polarisabilities that come at NNLO. With the exception of the proton $\alpha_{E 1}$ and $\beta_{M 1}$, they are calculated in the standard fashion (see e.g. $[1,42,48,49])$ by considering the convergence order-byorder in the chiral expansion. As discussed above, our expansion parameter is $\delta=m_{\pi} / \Delta=0.48$, with $m_{\pi} / \Lambda_{\chi} \sim \delta^{2}$. The LO contribution to the polarisabilities is from the $\pi \mathrm{N}$ loops, and the NLO contributions are from the $\Delta$ diagrams. A conservative estimate for the uncertainty on a given polarisability is therefore given by

$\sigma=\operatorname{Max}\left[\delta^{2} \times(\pi \mathrm{N}\right.$ loops $), \delta \times(\Delta$ graphs $), \delta^{2} \times($ total $\left.)\right]$

where of course it is the absolute values which are compared. The question of "theory errors" in effective theories has recently been considered from a Bayesian perspective by Furnstahl et al. [50]; briefly, the actual calculation of an observable to one or more orders tells us something about the size of the contributions, updating our prior expectations, e.g. of "naturalness" of the terms in the series. With minimal prior assumptions, the quantity obtained from the equivalent of Eq. (19) in a calculation to $k$ orders corresponds to the $k /(k+1)$ confidence limit. Hence in this case, with two orders calculated, $\sigma$ is a $67 \%$ confidence limit, very close to the conventional "one sigma" band. Furnstahl et al. also caution, however, that the corresponding probability distribution is in general quite non-Gaussian, and the "two sigma" interval will generally correspond to much less than a $95 \%$ probability interval.

The proton $\alpha_{E 1}$ and $\beta_{M 1}$ are a special case. Were we to treat them in the same way, we would obtain substantial errors of 3.1 and 2.7, respectively. However, alone of all the proton polarisabilities considered, these have counterterms at NNLO. In the absence of other information, one would expect their scale to be set by $\alpha_{\mathrm{em}} \Lambda_{\chi}^{-3} \sim 2.2$, which is entirely compatible with these errors. But we do have other information, since a partial NNLO fit to proton Compton-scattering data was performed by some of the current authors in Ref. [14], obtaining values for $\alpha_{E 1}$ and $\beta_{M 1}$ much closer to the NLO predictions than these uncertainties would suggest (see the penultimate line of Table 3). Although other, presumably similar-sized, NNLO mechanisms will enter in a full calculation, the counterterms in the fit will be readjusted and the net result is not expected to change significantly. This can be regarded as a vindication of the error estimate of \pm 0.7 on $\alpha_{E 1}$ and $\beta_{M 1}$ given in Ref. [13], and so we retain these errors in this work.

\section{Dynamical polarisabilities and multipoles}

\subsection{Definitions}

A widely used parametrisation of the Compton amplitude is the multipole expansion in the centre-of-mass frame. The initial and final photon-nucleon states in Compton scattering can be described using the angular momentum of the initial photon $l$, the index \pm for $j=l \pm 1$, and $E$ or $M$ to indicate initial and final photon parity: hence for each $l \geq 1$ there are four multipole amplitudes $f_{E E}^{l \pm}, f_{M M}^{l \pm}, f_{E M}^{l+}$ and $f_{M E}^{l+}$. As we are concerned only with the non-Born part of the Compton amplitude, the Born contribution is likewise implied to have been subtracted from the multipole amplitudes. The imaginary parts of the latter can be related to pion photoproduction multipoles via

$$
\begin{aligned}
& \operatorname{Im} f_{E E}^{l \pm}=k \sum_{c}\left|E_{(l \pm 1) \mp}^{(c)}\right|^{2}, \\
& \operatorname{Im} f_{M M}^{l \pm}=k \sum_{c}\left|M_{l \pm}^{(c)}\right|^{2} \\
& \operatorname{Im} f_{E M}^{l \pm}= \pm k \sum_{c} \operatorname{Re}\left[E_{(l \pm 1) \mp}^{(c)} M_{(l \pm 1) \mp}^{(c) *}\right] \\
& \operatorname{Im} f_{M E}^{l \pm}=\mp k \sum_{c} \operatorname{Re}\left[E_{l \pm}^{(c)} M_{l \pm}^{(c) *}\right] .
\end{aligned}
$$

Here, the sum is over the production charge channels (i.e., $\pi^{+} n$ and $\left.\pi^{0} p\right), k$ is the pion momentum in the centre-of-mass frame, and it is assumed that energy is sufficiently small so that only single pion production channel is significant. The dependence of each of the quantities in the r.h.s. and l.h.s. of these relations on the photon energy $\omega$ is implied. In particular, the leading low-energy behaviour of the multipoles is $[51,52]$

$$
\begin{aligned}
& f_{E E}^{l \pm} \sim f_{M M}^{l \pm} \sim \omega^{2 l} \\
& f_{E M}^{l+} \sim f_{M E}^{l+} \sim \omega^{2 l+1} .
\end{aligned}
$$

For the details of the multipole decomposition of the Compton amplitude, in particular, expressions for multipoles in terms of the amplitudes $A^{\mathrm{cm}}$, the reader is referred to Ref. [38] and references cited therein.

The multipole decomposition of the non-Born Compton amplitude allows one to introduce the dynamical nucleon 
polarisabilities $[38,53]$. The conventional definitions of these are

$$
\begin{gathered}
\alpha_{E l}(\omega)=(l(2 l-1) ! !)^{2} \frac{(l+1) f_{E E}^{l+}+l f_{E E}^{l-}}{\omega^{2 l}} \\
\beta_{M l}(\omega)=(l(2 l-1) ! !)^{2} \frac{(l+1) f_{M M}^{l+}+l f_{M M}^{l-}}{\omega^{2 l}}
\end{gathered}
$$

for the spin-independent dynamical polarisabilities, and

$$
\begin{gathered}
\gamma_{E l M(l+1)}(\omega)=2^{2-l}(2 l+1) ! ! \frac{f_{E M}^{l+}}{\omega^{2 l+1}}, \\
\gamma_{M l E(l+1)}(\omega)=2^{2-l}(2 l+1) ! ! \frac{f_{M E}^{l+}}{\omega^{2 l+1}}
\end{gathered}
$$

for the mixed spin dynamical polarisabilities. The unmixed spin dynamical polarisabilities are defined as

$$
\begin{gathered}
\gamma_{E l E l}(\omega)=(2 l-1) \frac{f_{E E}^{l+}-f_{E E}^{l-}}{\omega^{2 l+1}}, \\
\gamma_{M l M l}(\omega)=(2 l-1) \frac{f_{M M}^{l+}-f_{M M}^{l-}}{\omega^{2 l+1}} .
\end{gathered}
$$

The Compton multipoles (or, equivalently, the dynamical polarisabilities) capture the underlying physics in the different energy regimes and multipolarities (such as, e.g., the pion photoproduction cusp and the Delta peak). This provides an effective parameterisation of the Compton amplitude, working well in a wide range of energies $[38,54]$.

For $l=1$ the dynamical polarisabilities, defined in Eqs. (23-25), can be regarded as an extension of the static scalar and spin polarisabilities to be functions of the photon energy, with the latter matching the limiting values of the former as the energy goes to zero. However, the fact that the static polarisabilities are defined in the Breit frame causes this relation to be broken by the recoil terms for higher values of $l$. In fact, for $l \geq 2$ even the definition of the unmixed spin polarisabilities, Eq. (25), is problematic: while for $l=1$ the leading $\omega$ dependences cancel between the two unmixed multipoles, for $l \geq 2$ the recoil corrections fail to cancel, and cause the difference as well as the weighted sum to go as $\omega^{2 l}$. This means that the unmixed $l \geq 2$ spin dynamical polarisabilities diverge as $1 / \omega$ in the zero-energy limit. In spite of this limitation, the (non-divergent) dynamic polarisabilities provide a compact way to summarise the amplitudes and to compare the predictions of different frameworks.

As argued above, the static polarisabilities are best defined via the effective non-relativistic Hamiltonian in the Breit frame, and not as the zero-energy limit of the dynamical polarisabilities. However, it is straightforward to calculate the relevant recoil corrections in the centre-of-mass frame and hence match the low-energy expansion of the dynamical polarisabilities to the static ones. Details of this calculation are given in Appendix B; here we only note one result for the zero-energy limit of the scalar quadrupole polarisabilities [34]:

$\alpha_{E 2}(0)=\alpha_{E 2}+\frac{3 \beta_{M 1}}{2 M_{\mathrm{N}}^{2}} \quad \beta_{M 2}(0)=\beta_{M 2}+\frac{3 \alpha_{E 1}}{2 M_{\mathrm{N}}^{2}}$.

\subsection{Results}

Here we provide predictions for the proton and neutron dynamical polarisabilities and Compton multipoles; our results are shown in Figs. 6, 7, 8, 9, 10, 11, 12 and 13. Figures 6 and 7 show the proton scalar (dipole and quadrupole) and spin dipole dynamical polarisabilities, in order. We compare our results with the dispersive calculation of Ref. [38] and the results of the Computational Hadronic Model (CHM) [55,56] (this framework is based on a covariant $\chi \mathrm{PT}$, and the main difference between our calculation and that of the CHM is the treatment of the Delta isobar; cf. the discussion below). Note that the DR calculation of Ref. [38] has been constrained to reproduce the values of $\alpha_{E 1}$ and $\beta_{M 1}$ of Refs. [37] and [44] for the proton and the neutron, respectively; see Tables 3 and 5. In addition, we show the effect of the loop corrections to the $\gamma N \Delta$ vertex.

The uncertainty bands in these figures are generated with a similar method to that used for the static polarisabilities, in particular, at low energies these bands are defined by the corresponding uncertainties on the static polarisabilities $\sigma$ [see Eq. (19)]. At higher energies we estimate the errors due to higher-order contributions as

$$
\begin{aligned}
\sigma(\omega)= & \operatorname{Max}[\delta \times(\Delta \text { pole corr. }), \delta \times(\pi \mathrm{N} \text { loops }), \\
& \delta \times(\pi \Delta \text { loops }),(\text { sum })]
\end{aligned}
$$

with $\delta=\omega / \Lambda_{\chi}$ and the fourth term being the sum of the first three. Besides that, since the shape of the Delta peak is well constrained and is well reproduced in $\chi \mathrm{PT}$, higher-order corrections would be expected to change mostly its magnitude, in which case they can be absorbed by means of fitting the $\gamma N \Delta$ coupling constants to experimental data. We therefore treat the leading Delta-pole contribution differently in this regime, estimating the uncertainty $\sigma_{g_{M}}(\omega)$ of the dynamic polarisability from this contribution by varying $g_{M}$ by \pm 0.1 .

The final uncertainty band is calculated at any energy by taking the largest of these quantities: $\operatorname{Max}\left[\sigma, \sigma(\omega), \sigma_{g_{M}}(\omega)\right]$. Note that the nucleon Born graphs, which do not enter the dynamical polarisabilities but contribute to the Compton multipoles (and the observables), are also well constrained and so are not taken into account in the uncertainty estimates.

Only in those polarisabilities and multipoles that receive a significant contribution from the Delta pole and only at energies around the Delta peak is the uncertainty driven by 

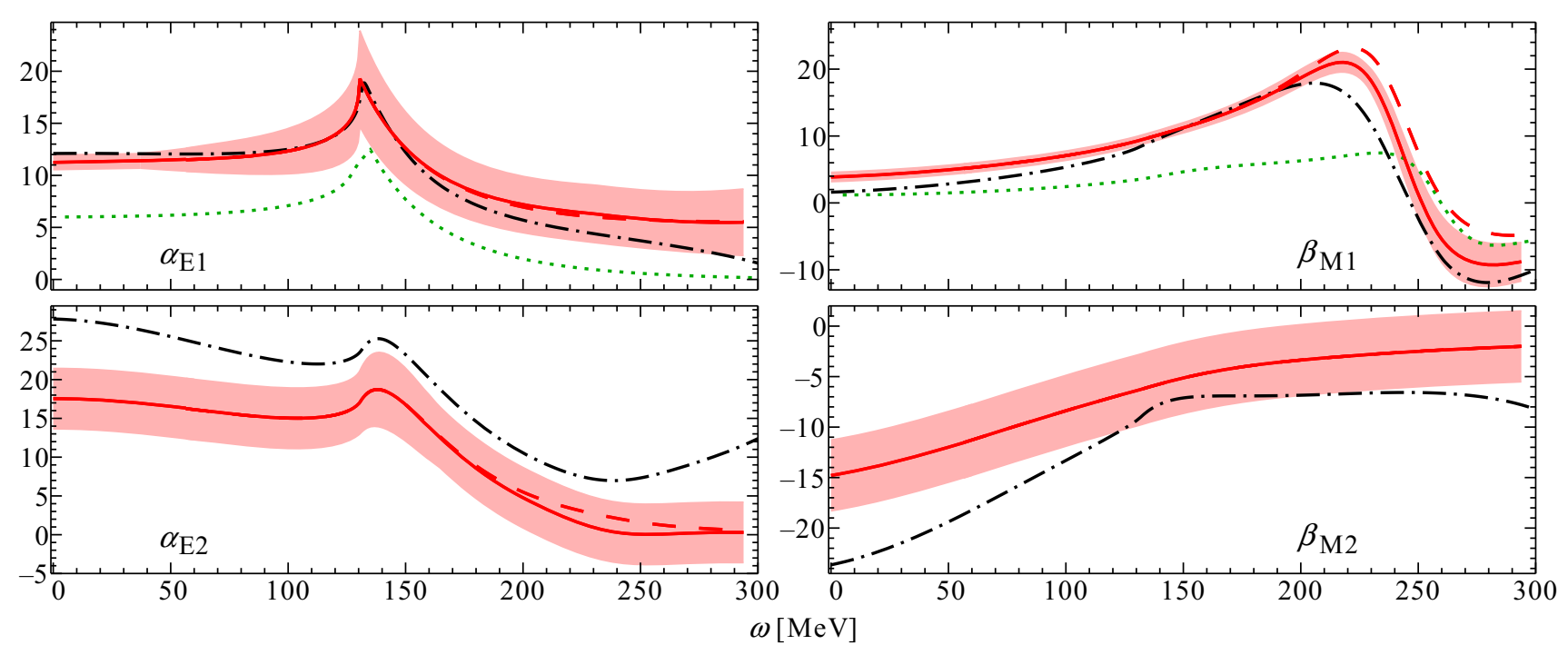

Fig. 6 Real parts of proton scalar dipole and quadrupole dynamical polarisabilities, in units of $10^{-4} \mathrm{fm}^{3}$ and $10^{-4} \mathrm{fm}^{5}$, respectively, as functions of photon cms energy in $\mathrm{MeV}$. The curves are the results of this $\mathrm{B} \chi \mathrm{PT}$ calculation with or without the $\gamma N \Delta$ vertex running (red

solid and red dashed, respectively), compared with the results of the DR calculation of Ref. [38] (black dot-dashed, curves from Ref. [39]) and with the results of the Computational Hadronic Model (CHM) [55,56] (green dotted, not shown for the quadrupole polarisabilities)
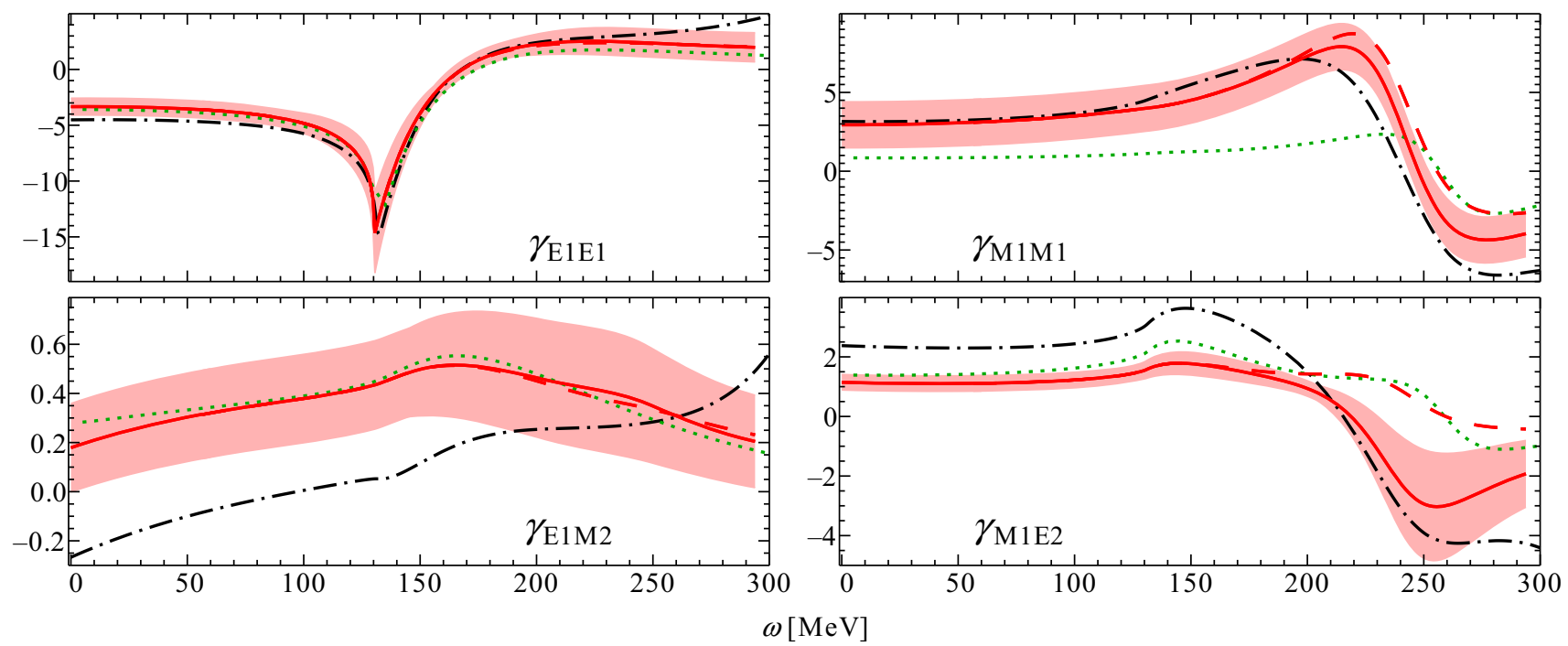

Fig. 7 Real parts of proton spin dynamical polarisabilities in units of $10^{-4} \mathrm{fm}^{4}$ as functions of the photon cms energy in MeV. The curves are the results of this $\mathrm{B} \chi \mathrm{PT}$ calculation with or without the $\gamma N \Delta$ vertex running (red solid and red dashed, respectively), compared with

the results of the DR calculation of Ref. [38] (black dot-dashed, curves from Ref. [39]) and with the results of the Computational Hadronic Model [55,56] (green dotted)

$\sigma(\omega)$ or $\sigma_{g_{M}}(\omega)$, otherwise it coincides with the static uncertainty. The only notable exception from this rule are the proton $\alpha_{E 1}(\omega)$ and $\beta_{M 1}(\omega)$ - these two polarisabilities have been assigned small static uncertainties, and the energy-dependent uncertainty estimate exceeds them at relatively low energies, cf. Fig. 6.

One can see from Figs. 6 and 7 that our results for the dynamical polarisabilities agree qualitatively with the dispersive calculation of Ref. [38]. There are, however, some differences, for instance, our scalar polarisability $\beta_{M 1}(\omega)$ as well as the spin polarisabilities $\gamma_{M 1 M 1}(\omega)$ and $\gamma_{M 1 E 2}(\omega)$ show sizeable deviation from the DR curves, both at low energies and in the region of the Delta peak. The inclusion of the loop corrections to the $\gamma N \Delta$ vertex moves our curves closer to the DR ones, especially at energies around the Delta pole; this was first noted in Ref. [57]. The remaining difference, especially the $\mathrm{B} \chi \mathrm{PT}$ polarisabilities being rather high around the Delta peak, might be explained, as speculated in Ref. [14], if the leading $\gamma N \Delta$ coupling constant is a bit too large. One could fit this constant to see if a closer agreement 

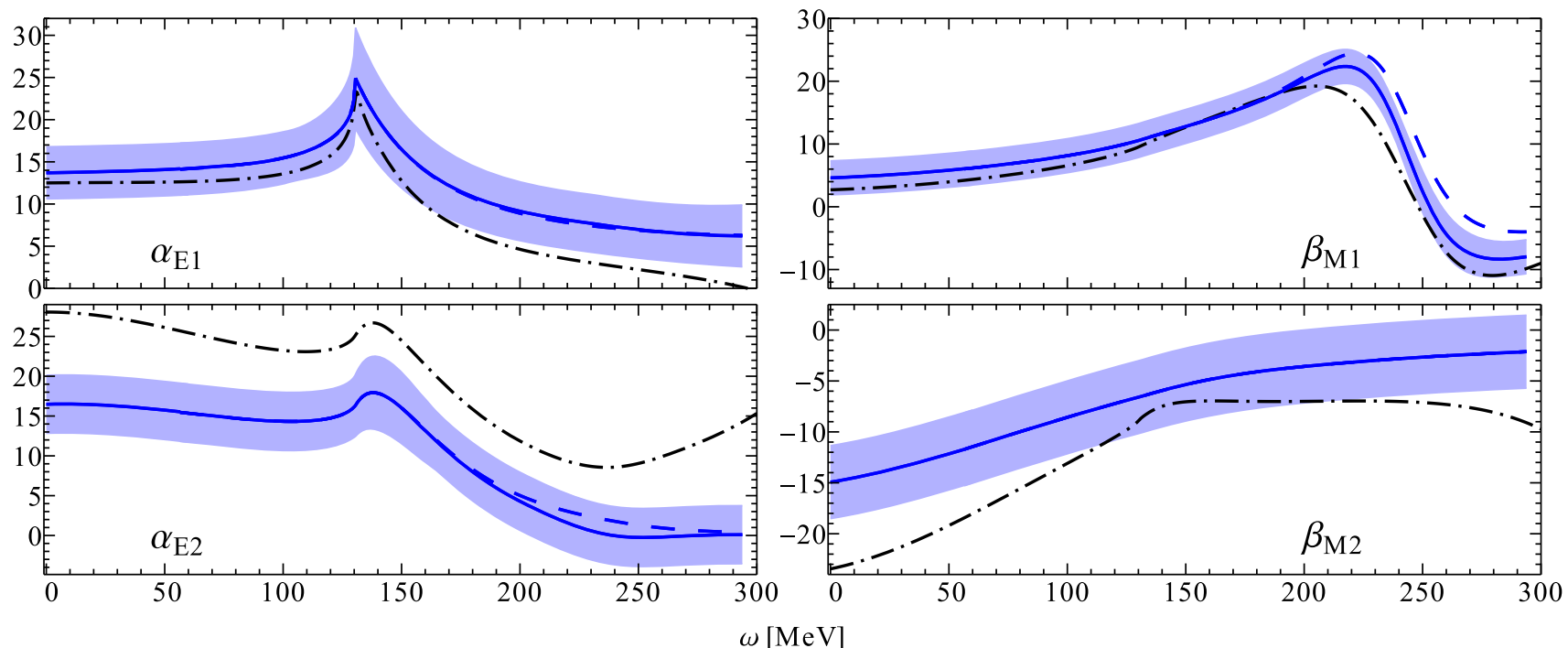

Fig. 8 Real parts of neutron scalar dipole and quadrupole dynamical polarisabilities, in units of $10^{-4} \mathrm{fm}^{3}$ and $10^{-4} \mathrm{fm}^{5}$, respectively, as

this $\mathrm{B} \chi \mathrm{PT}$ calculation with or without the $\gamma N \Delta$ vertex running (blue solid and blue dashed, respectively), compared with the results of the DR calculation of Ref. [38] (black dot-dashed, curves from Ref. [39])
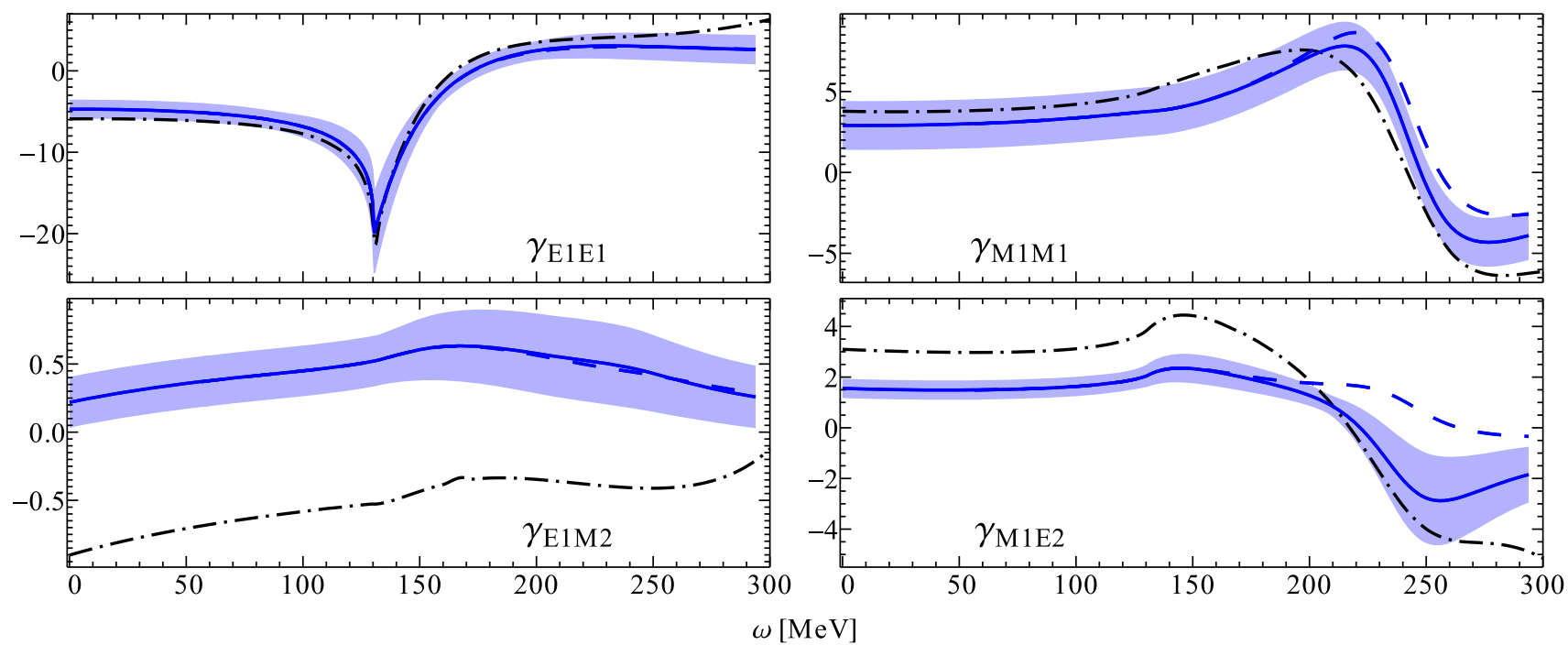

Fig. 9 Real parts of neutron spin dynamical polarisabilities in units of $10^{-4} \mathrm{fm}^{4}$ as functions of the photon c.m. energy in MeV. The curves are the results of this $\mathrm{B} \chi \mathrm{PT}$ calculation with or without the $\gamma N \Delta$ ver-

tex running (blue solid and blue dashed, respectively), compared with the results of the DR calculation of Ref. [38] (black dot-dashed, curves from Ref. [39])

with the Compton data results at the same time in a better agreement between the $\chi \mathrm{PT}$ and DR calculations. Overall, the $\mathrm{B} \chi \mathrm{PT}$ calculation reproduces all the characteristic features that show up in the DR calculations, such as the pion production cusp and the Delta peak, which is not surprising given the physics contents of the chiral Lagrangians used in the $\chi$ PT calculations.

The Computational Hadronic Model, on the other hand, is close to $\mathrm{B} \chi \mathrm{PT}$ in those polarisabilities where the Delta pole does not contribute much to the energy dependence: $\alpha_{E 1}(\omega)$, $\gamma_{E 1 E 1}(\omega)$ and $\gamma_{E 1 M 2}(\omega)$. In $\alpha_{E 1}(\omega)$ the difference can be

explained by the fact that the curves of Ref. [55,56] do not contain the $\pi \Delta$ loops whose contribution is nearly a constant function of $\omega[15,38])$. The CHM tends, however, to substantially underpredict the Delta-pole contribution to the Deltadominated dynamical polarisabilities, especially $\beta_{M 1}(\omega)$ and $\gamma_{M 1 M 1}(\omega)$.

The treatment of the Delta isobar in the CHM differs from ours as follows: the $\gamma N \Delta$ Lagrangian used in the former calculation contains only the LO term (corresponding to our $g_{M}$ coupling), and this LO Lagrangian does not possess the spin-3/2 gauge symmetry that ensures the spurious spin- $1 / 2$ 

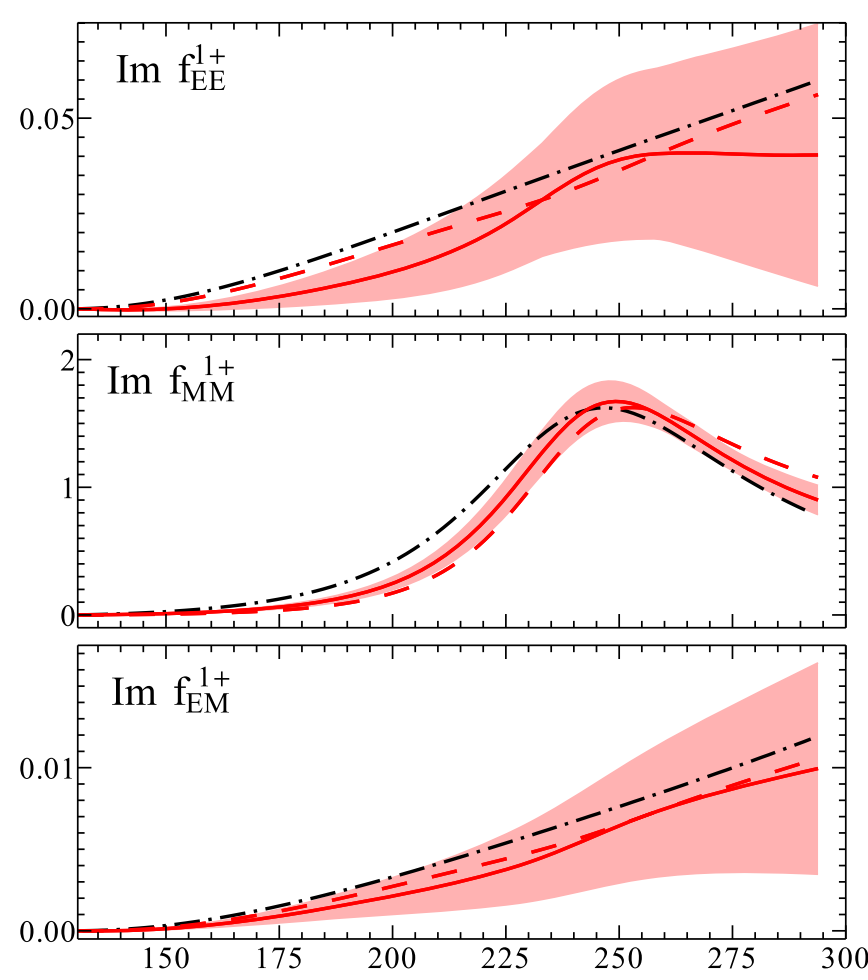

$\omega[\mathrm{MeV}]$

Fig. 10 Imaginary parts of $l=1$ proton Compton multipoles in units of $10^{-3} \mathrm{~m}_{\pi}^{-1}$ as functions of the photon c.m. energy in MeV. The curves are the results of this $\mathrm{B} \chi \mathrm{PT}$ calculation, with or without the $\gamma N \Delta$ vertex
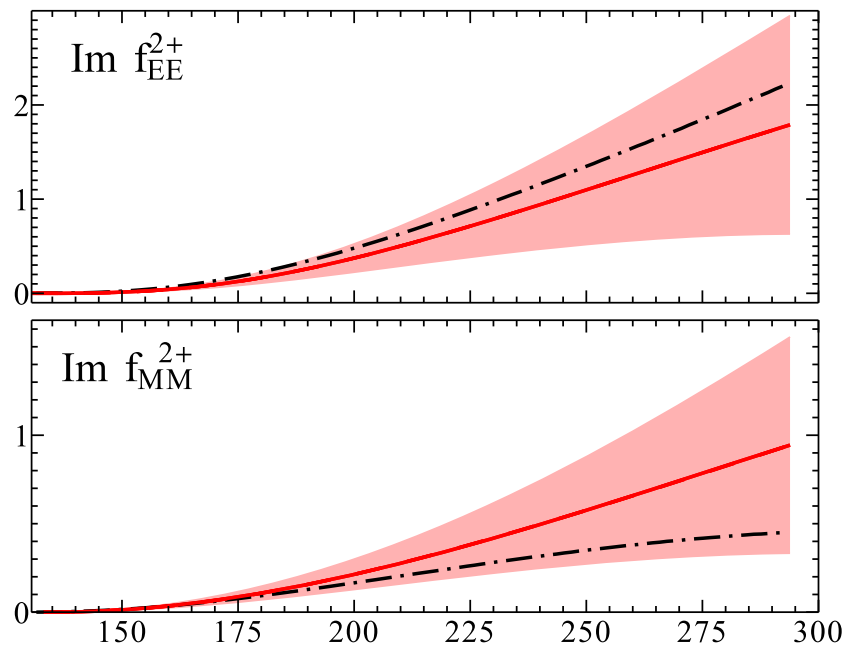

$\omega[\mathrm{MeV}]$

Fig. 11 Imaginary parts of $l=2$ proton Compton multipoles in units of $10^{-6} \mathrm{~m}_{\pi}^{-1}$ as functions of the photon c.m. energy in $\mathrm{MeV}$. The curves are the results of this $\mathrm{B} \chi \mathrm{PT}$ calculation with or without the $\gamma N \Delta$ vertex

degrees of freedom do not contribute in the amplitudes containing the Delta (see the discussion in Ref. [10] and references therein). In addition, the calculation of the CHM does not include pion loops with the Delta isobar. Of these differences, only the admixture of a spin- $1 / 2$ component in the
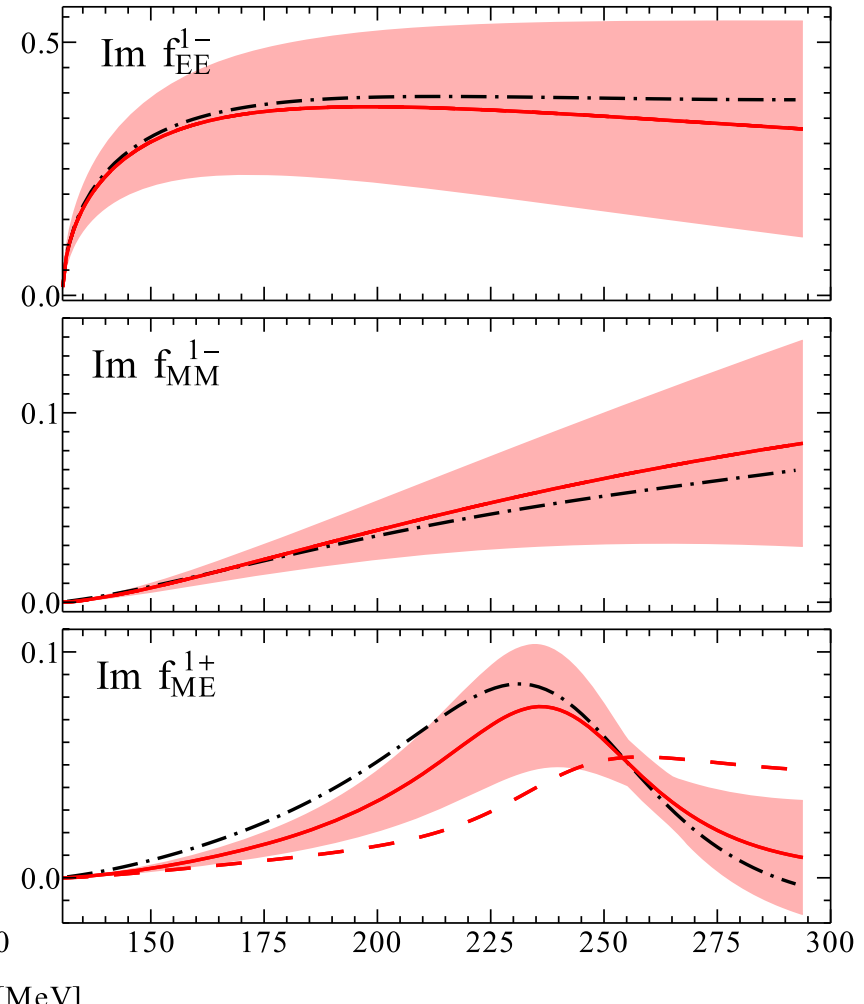

running (red solid/red dashed, respectively), compared with the results of the MAID pion photoproduction analysis [58] (black dot-dashed)
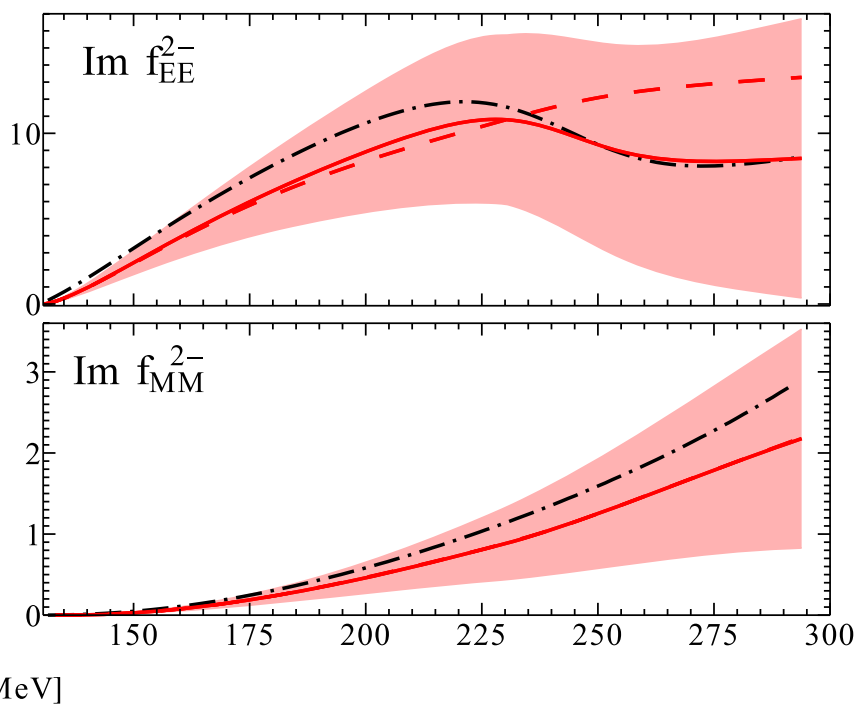

running (red solid/red dashed, respectively), compared with the results of the MAID pion photoproduction analysis [58] (black dot-dashed)

Delta isobar has the potential to account for the difference in the results for $\beta_{M 1}(\omega)$ and $\gamma_{M 1 M 1}(\omega)$; it remains to be seen, however, whether this is in fact the reason behind the underestimated Delta-driven polarisabilities in the calculation of Refs. [55,56]. 

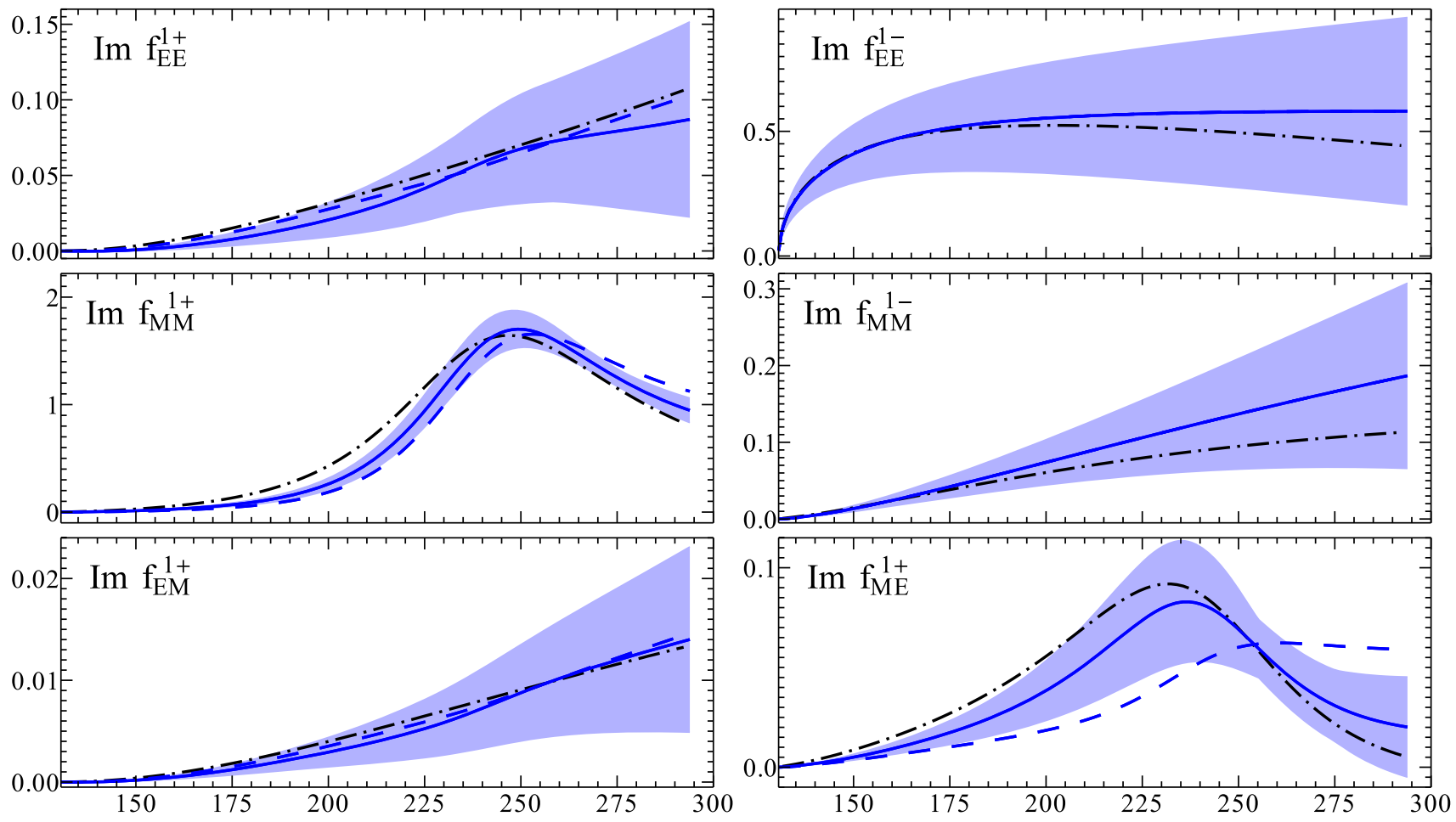

$\omega[\mathrm{MeV}]$

Fig. 12 Imaginary parts of $l=1$ neutron Compton multipoles in units of $10^{-3} \mathrm{~m}_{\pi}^{-1}$ as functions of the photon c.m. energy in MeV. The curves are the results of this $\mathrm{B} \chi \mathrm{PT}$ calculation, with or without the $\gamma N \Delta$ ver-
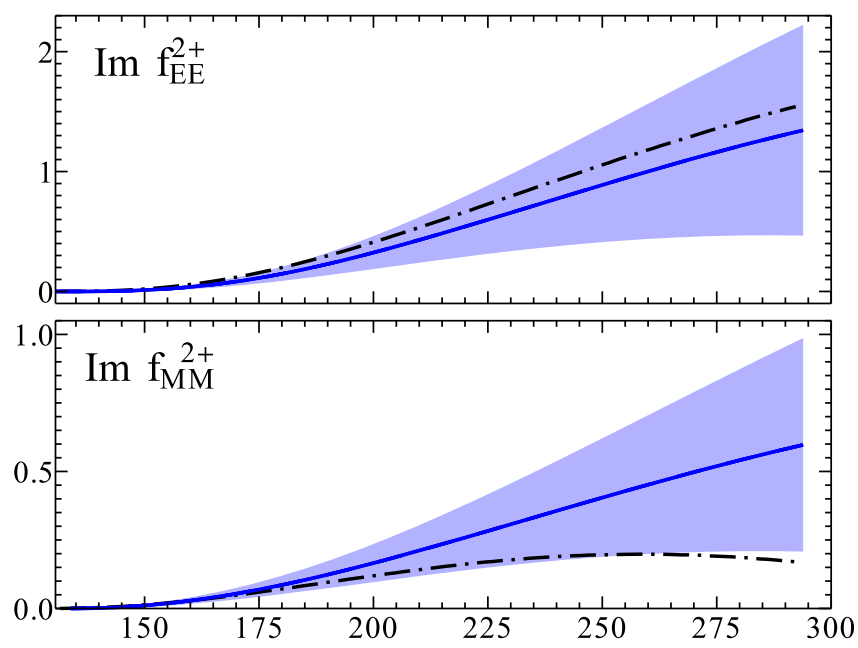

$\omega[\mathrm{MeV}$

Fig. 13 Imaginary parts of $l=2$ neutron Compton multipoles in units of $10^{-6} \mathrm{~m}_{\pi}^{-1}$ as functions of the photon cms energy in $\mathrm{MeV}$. The curves are the results of this $\mathrm{B} \chi \mathrm{PT}$ calculation with or without the $\gamma N \Delta$ ver-

The theoretical uncertainty bands, together with the curves that do not contain the $\gamma N \Delta$ vertex corrections (which are NLO at $\omega \sim \Delta$ ), illustrate the estimated contribution of the terms that can enter at the next order. $\gamma_{E 1 M 2}(\omega)$ has the largest fractional uncertainty, because its small central value arises tex running (blue solid/blue dashed, respectively), compared with the results of the MAID pion photoproduction analysis [58] (black dotdashed)
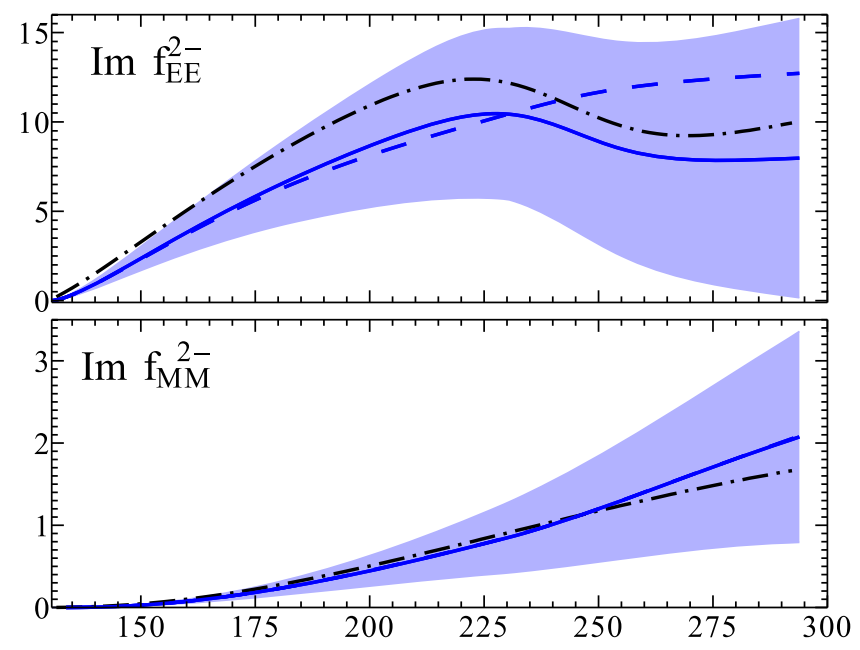

tex running (blue solid/blue dashed, respectively), compared with the results of the MAID pion photoproduction analysis [58] (black dotdashed)

from cancellations between the leading and subleading contributions.

In Figs. 8 and 9 we show the same polarisabilities as above in Figs. 6 and 7, respectively, but now for the neutron. They are compared with the neutron results of the DR calculation 
of Ref. [38], and we also show in these figures the effect of the loop correction to the $\gamma N \Delta$ vertex, analogously to the proton case. Our results for the neutron demonstrate a qualitative agreement with the DR calculation comparable to that observed above for the proton. This is not surprising: as pointed out above, all the essential physics included in the DR is also captured by the chiral calculation at this order. The main features of the neutron curves are the same as have been described above for the case of the proton. In particular, the most significant deviations between the $\mathrm{B} \chi \mathrm{PT}$ and the $\mathrm{DR}$ curves around the Delta peak occur in the scalar magnetic dipole polarisability $\beta_{M 1}(\omega)$ and in the two spin polarisabilities, $\gamma_{M 1 M 1}(\omega)$ and $\gamma_{M 1 E 2}(\omega)$, where the Delta contribution is dominant. It is also noticeable that the DR values of the neutron $\gamma_{E 1 M 2}(\omega)$ are significantly different from the proton ones, whereas the chiral calculation at this order gives essentially the same values for the proton and the neutron. This difference makes the disagreement between the DR and $\mathrm{B} \chi \mathrm{PT}$ curves for the neutron $\gamma_{E 1 M 2}(\omega)$ more prominent than in the proton case.

Moving from dynamical polarisabilities to Compton multipoles, we show in Figs. 10 and 11 our results for the imaginary parts of the proton multipoles with $l=1$ and $l=2$, respectively. The analogous neutron curves are shown in Fig. 12 for the imaginary parts of the $l=1$ multipoles and in Fig. 13 for those with $l=2$. They are compared with the results of the MAID multipole analysis [58] of pion photoproduction, converted into the imaginary parts of the Compton multipoles via Eqs. (20-21). The error bands are calculated as explained above, and we again show the effect of the loop corrections to the $\gamma N \Delta$ vertex. Our results agree reasonably well with the MAID curves - the latter lie within the calculated uncertainty bands in most cases for both the proton and the neutron. It can also be seen that the inclusion of the $\gamma N \Delta$ vertex corrections improves the agreement with the MAID analysis in most cases. In some multipoles, however, there is a slight disagreement between the $\mathrm{B} \chi \mathrm{PT}$ results and the MAID analysis, most notably, in the leading slope of the resonance peak in $\operatorname{Im} f_{M M}^{1+}(\omega)$ and in $\operatorname{Im} f_{M E}^{1+}(\omega)$ as well as in $\operatorname{Im} f_{E E}^{1+}(\omega)$ where the addition of the loop corrections actually worsens the agreement between the $\mathrm{B} \chi \mathrm{PT}$ and the MAID curves, particularly for the proton.

\section{Polarised observables for the proton}

The wealth of world data on unpolarised Compton scattering has allowed for high-precision extractions of $\alpha_{E 1}$ and $\beta_{M 1}$ in chiral EFT fits, for instance, in a $\mathscr{O}\left(p^{4}\right) \mathrm{HB} \chi \mathrm{PT}$ fit [11], and a partial $\mathscr{O}\left(p^{4}\right) \mathrm{B} \chi \mathrm{PT}$ fit [14] based on the current calculation. In contrast, the unpolarised cross section appears to be very weakly sensitive to the spin polarisabilities: even though their values differ significantly in $\mathrm{HB} \chi \mathrm{PT}$ and in the current $\mathrm{B} \chi \mathrm{PT}$ work, the resulting fits to the unpolarised Compton cross section yield practically identical values of $\alpha_{E 1}-\beta_{M 1}$ [14]. On the other hand, (double) polarised observables, in particular, the beam asymmetry $\Sigma_{3}$, and the beam-target asymmetries $\Sigma_{2 x}$ and $\Sigma_{2 z}$ are known to be sensitive to the values of the spin polarisabilities [34]. These asymmetries are defined as follows.

$\Sigma_{3}$ is the beam asymmetry for photons polarised linearly either parallel or perpendicularly to the scattering plane, with nucleons unpolarised:

$\Sigma_{3}=\frac{\mathrm{d} \sigma^{\|}-\mathrm{d} \sigma^{\perp}}{\mathrm{d} \sigma^{\|}+\mathrm{d} \sigma^{\perp}}$

$\Sigma_{2 x}$ is the beam-target asymmetry for photons polarised circularly and nucleons polarised along the $x$ axis, i.e., in the reaction plane perpendicularly to the photon momentum (which is along the $z$ axis):

$\Sigma_{2 x}=\frac{\mathrm{d} \sigma_{x}^{R}-\mathrm{d} \sigma_{x}^{L}}{\mathrm{~d} \sigma_{x}^{R}+\mathrm{d} \sigma_{x}^{L}}$

and $\Sigma_{2 z}$ is beam-target asymmetry for photons polarised circularly and nucleons polarised along the $z$ axis:

$\Sigma_{2 z}=\frac{\mathrm{d} \sigma_{z}^{R}-\mathrm{d} \sigma_{z}^{L}}{\mathrm{~d} \sigma_{z}^{R}+\mathrm{d} \sigma_{z}^{L}}$.

In addition, we consider $\Sigma_{1 z}$, the beam-target asymmetry for photons polarised linearly with the polarisation directed under $\pm \pi / 4$ with respect to the reaction plane and nucleons polarised along the $z$ axis:

$\Sigma_{1 z}=\frac{\mathrm{d} \sigma_{z}^{\pi / 4}-\mathrm{d} \sigma_{z}^{-\pi / 4}}{\mathrm{~d} \sigma_{z}^{\pi / 4}+\mathrm{d} \sigma_{z}^{-\pi / 4}}$.

The latter asymmetry vanishes below the pion production threshold. The expressions for these asymmetries are given, e.g., in Ref. [34]; though the authors of that reference use a different tensor basis in the decomposition of the Compton amplitude, their expressions can easily be transformed to the basis used in our work; see Appendix A (see also Refs. [54, 59] for explicit expressions in terms of amplitudes $A_{i}^{\mathrm{cm}}$ ).

As shown in Ref. [34], measurement of the double polarised observables $\Sigma_{2 x}$ and $\Sigma_{2 z}$ can provide information on all four spin polarisabilities, by analysing the angular dependence of the asymmetries. In this section, we show our predictions for the asymmetries $\Sigma_{3}, \Sigma_{2 x}$, and $\Sigma_{2 z}$, together with theoretical uncertainty bands, and compare them with the data from LEGS [60] and with the recent data from Mainz [18,61]. We also provide our predictions for $\Sigma_{1 z}$ and discuss its features. For the sake of completeness, we 
also show our predictions for the unpolarised cross section. Dispersion-relation predictions for these asymmetries have been provided in Ref. [41].

The uncertainty bands shown in figures are obtained similarly to those calculated for the (real parts of) Compton multipoles, namely, adding to the Compton amplitude any of the quantities in Eq. 27,

$$
\begin{aligned}
\delta A & =\{\delta \times(\Delta \text { pole corr }), \delta \times(\pi \mathrm{N} \text { loops }), \\
\delta & \times(\pi \Delta \text { loops }),(\text { sum })\}
\end{aligned}
$$

then calculating the observables using the shifted amplitudes $A \pm \delta A$ and taking the one of the $\delta A$ 's that provides the maximal width of the band. Our estimate results in moderately wide uncertainty bands for all of the asymmetries, especially in $\Sigma_{2 z}$ and $\Sigma_{2 x}$. The band for the cross sections is also rather wide. Since the amplitude is dominated at these energies by the Delta pole, the main effect of adding the uncertainties as prescribed by Eq. (32) is to change the normalisation of the predicted cross sections - this change will mostly cancel in the asymmetries. By analogy, it also follows that some of the latter might be more sensitive to the mechanisms other than the Delta-pole or Born graphs.

In Fig. 14, we show the theoretical curves and bands for the unpolarised cross section, together with experimental data from many different experiments; see Table 3.1 of Ref. [1] for the list. This figure shows that the $\mathrm{B} \chi \mathrm{PT}$ prediction for the Compton unpolarised cross section is consistent with the available data at energies up to the Delta peak. We also include curves with only the nucleon Born and the Delta-pole graphs included, demonstrating the effect of the $\pi \mathrm{N}$ and $\pi \Delta$ loops on the unpolarised cross section.

Our predictions for $\Sigma_{3}$ are shown in Fig. 15, where we provide a comparison with the LEGS experimental data [60], at lab energies $E_{\gamma}$ in the range 220-340 MeV. We also show the recent data on this asymmetry from MAMI [61]. Our results are consistent with the LEGS data, especially at higher energies in this range. At lower energies the description of the data is a bit worse but still broadly satisfactory. The comparison between the dashed ans solid curves shows that the $\pi \mathrm{N}$ and $\pi \Delta$ loops also become more important at lower energies, especially since $\Sigma_{3}$ passes through zero at $E_{\gamma} \simeq 250 \mathrm{MeV}$. This view can also be supported by Fig. 18 (left panel) where we show $\Sigma_{3}$ as a function of $E_{\gamma}$ at $\theta=90^{\circ}$ : the data, in particular, their energy dependence, are well reproduced by the theoretical curve.

Figures 16 and 17 show the other two asymmetries, $\Sigma_{2 z}$ and $\Sigma_{2 x}$, respectively, at the same values of $\omega_{\text {lab }}$ as in Fig. 15. As pointed out above, uncertainty bands for these observables are narrower than for $\Sigma_{3}$.

Figure 16 includes the empirical extraction of $\Sigma_{2 z}$ at $\theta=0^{\circ}$ performed via evaluation of the forward sum rule in Ref. [62]. The $\mathrm{B} \chi \mathrm{PT}$ theoretical curves show a remark-

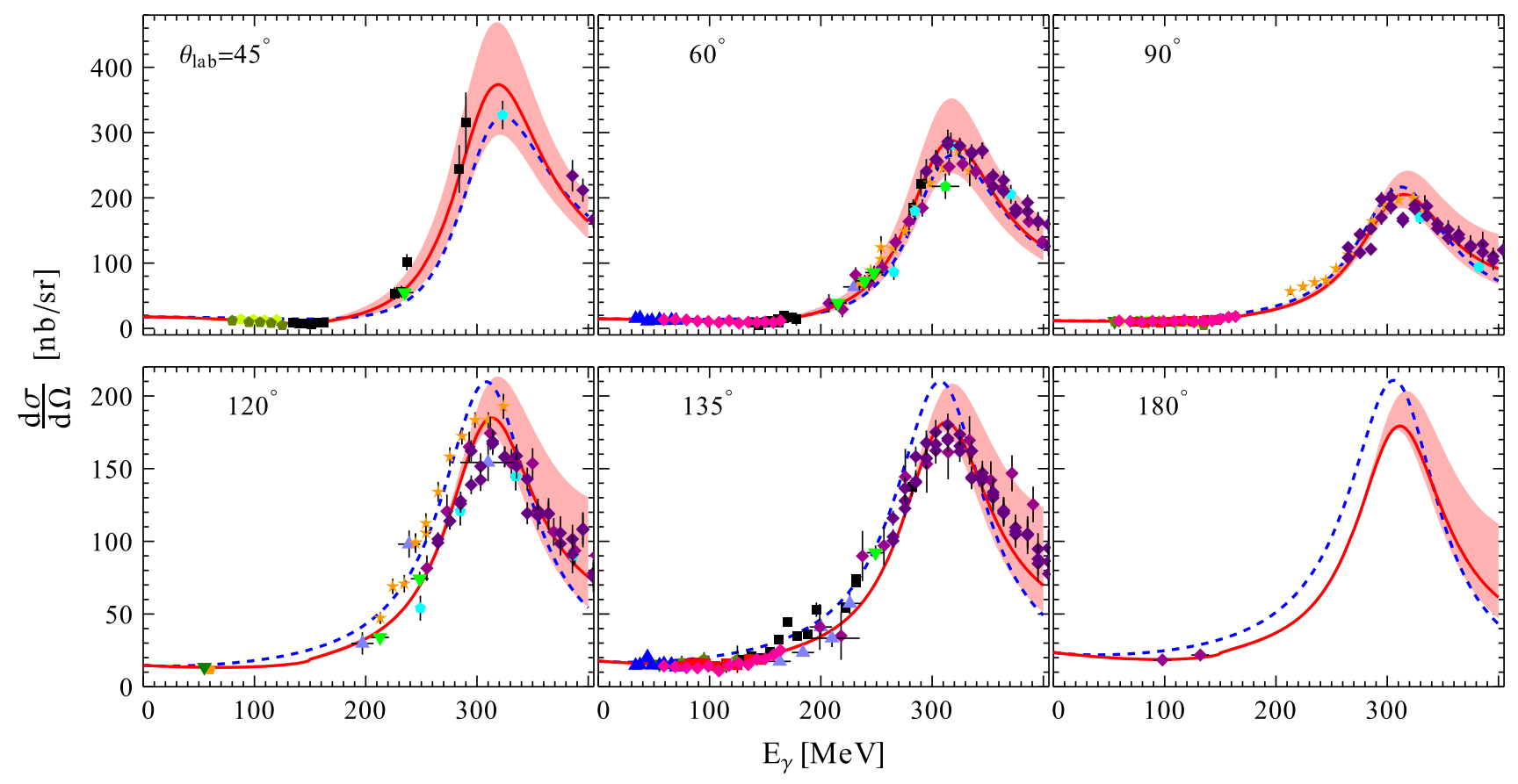

Fig. 14 Unpolarised differential Compton-scattering cross section in $\mathrm{nb} / \mathrm{sr}$ as a function of the photon energy $E_{\gamma}$ at fixed values of the laboratory frame scattering angle $\theta_{\text {lab. The curves }}$ are the complete results

of this $\mathrm{B} \chi \mathrm{PT}$ calculation (red solid) and the contribution of only the nucleon Born and Delta-pole graphs (blue dashed). For the data symbols see Table 3.1 of Ref. [1] 

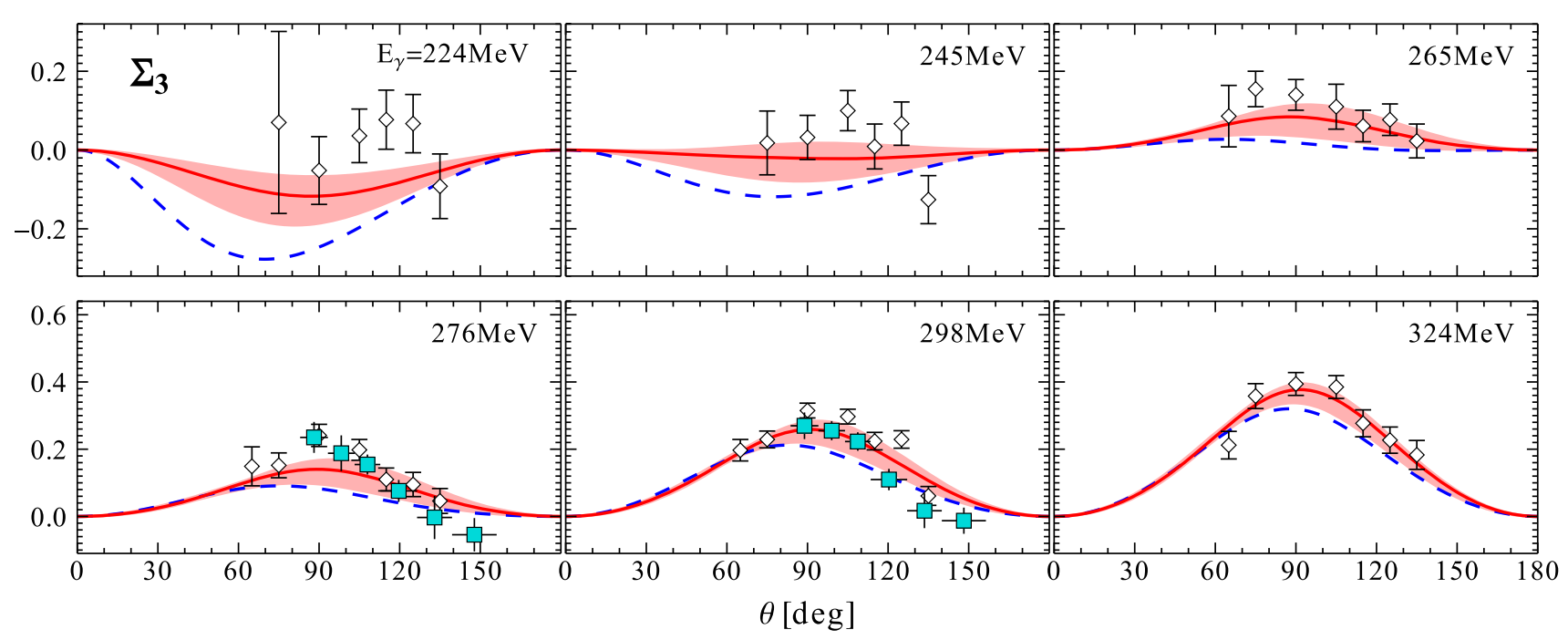

Fig. 15 Reaction asymmetry $\Sigma_{3}$ as a function of the c.m. angle at different values of $E_{\gamma}$ (annotated values are in $\mathrm{MeV}$ ), compared with data from LEGS [60] (open diamonds) and MAMI [61] (cyan squares). The theoretical bands correspond to the full calculation and their width is determined as explained in the text. The blue dashed lines correspond to only the Born + Delta-pole graphs included in the calculation
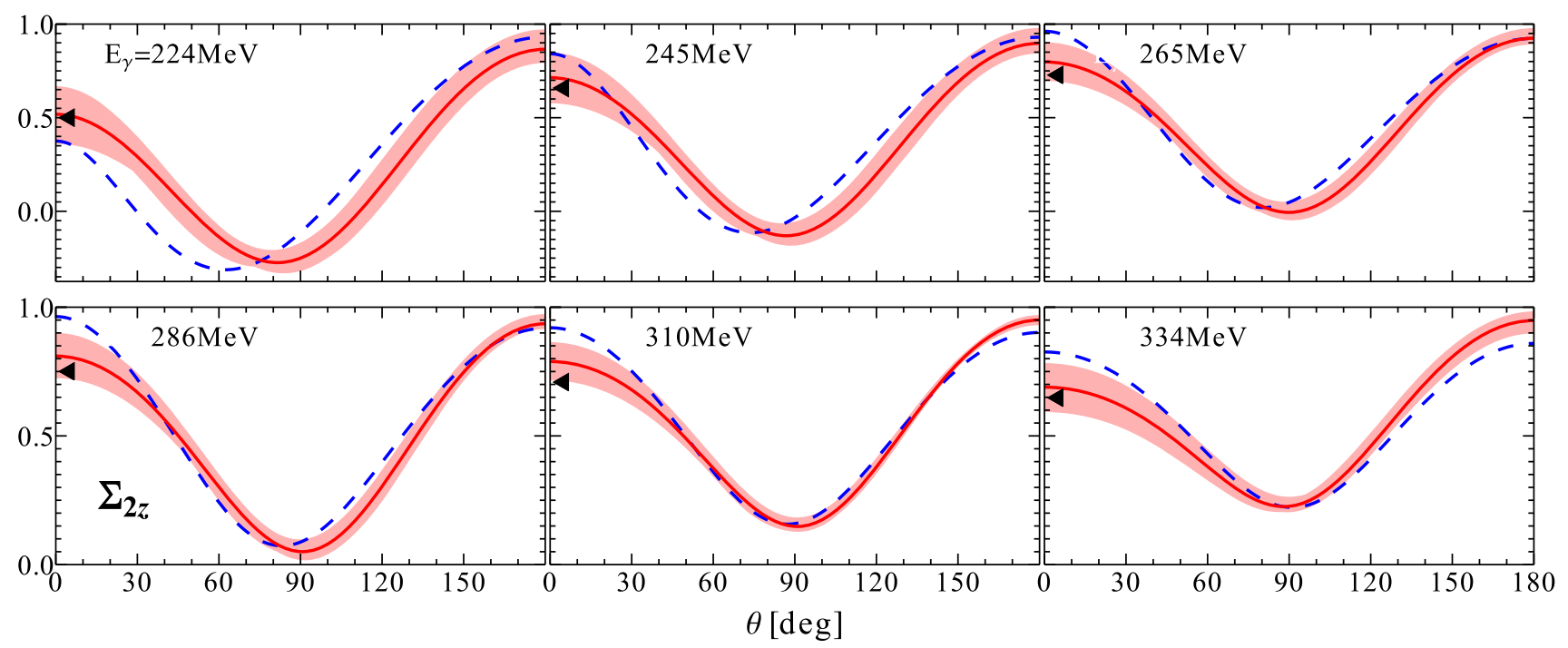

Fig. 16 Reaction asymmetry $\Sigma_{2 z}$ as a function of the cms angle at the same values of $E_{\gamma}$ as in Fig. 15 (annotated values are in $\mathrm{MeV}$ ). The black triangles show the results of the forward sum rule evaluation of Ref. [62]. The theoretical bands correspond to the full calculation and their width is determined as explained in the text. The blue dashed lines correspond to only the Born + Delta-pole graphs included in the calculation

prediction also describes these new data on $\Sigma_{2 x}$ well, the estimated theoretical uncertainty band being considerably smaller than the experimental errors. The typical width of the band is comparable to the variation of the theoretical curve with energy over the A2 experimental range given above. Again, the curves that include only the nucleon Born and Delta-pole graphs illustrate the relative importance of these and of the loop graphs in these asymmetries. One can see that the loop graphs generally become more important at lower energies, in accordance to what one would expect given that the Delta-pole amplitude, dominating 

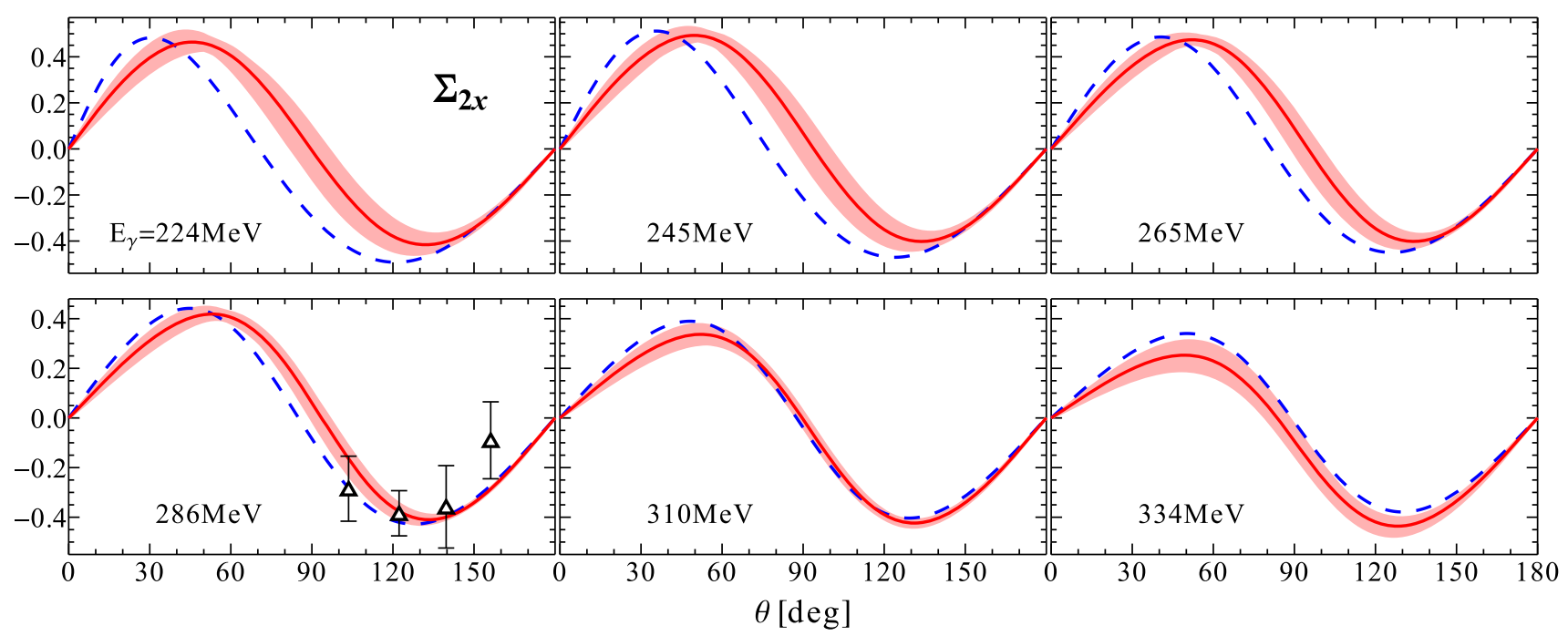

Fig. 17 Reaction asymmetry $\Sigma_{2 x}$ as a function of the c.m. angle at the same values of $\omega_{\mathrm{lab}}$ as in Fig. 15 (annotated values are in $\mathrm{MeV}$ ). The data (open triangles) at $E_{\gamma}=286 \mathrm{MeV}$ are from the recent A2 experiment [18]. The theoretical bands correspond to the full calcula-

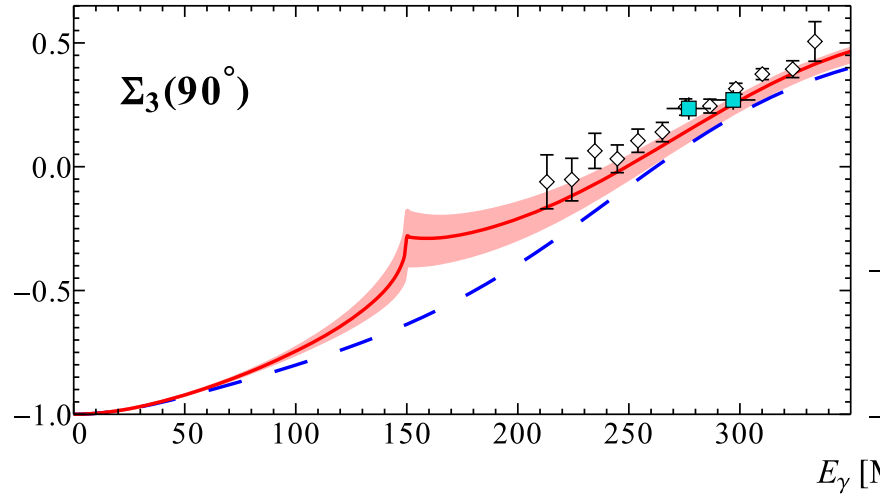

Fig. 18 Left panel reaction asymmetry $\Sigma_{3}$ as function of the laboratory frame photon energy at $\theta_{\mathrm{cm}}=90^{\circ}$, compared with the data from LEGS [60] (open diamonds) and MAMI [61] (cyan squares). Right panel reaction asymmetry $\Sigma_{1 z}$ as a function of the laboratory frame

around the Delta peak, quickly falls off as the photon energy decreases.

Last but not least, the right panel of Fig. 18 shows the predictions for the asymmetry $\Sigma_{1 z}$ at $\theta=90^{\circ}$ as a function of the lab frame photon energy. As pointed out above, $\Sigma_{1 z}=$ 0 below the pion production threshold. It is noticeable that this asymmetry is generated mostly by the imaginary part of pion loops - the Delta-pole contribution becomes important only at energies around $220 \mathrm{MeV}$ and higher. Note also that this asymmetry can be measured in the same experiment as $\Sigma_{3}$. Measuring this asymmetry in the region just above the pion production threshold could thus be done simultaneously with the planned measurements of $\Sigma_{3}$, and it has a potential to provide new important information on the nucleon pion cloud. tion and their width is determined as explained in the text. The blue dashed lines correspond to only the Born + Delta-pole graphs included in the calculation

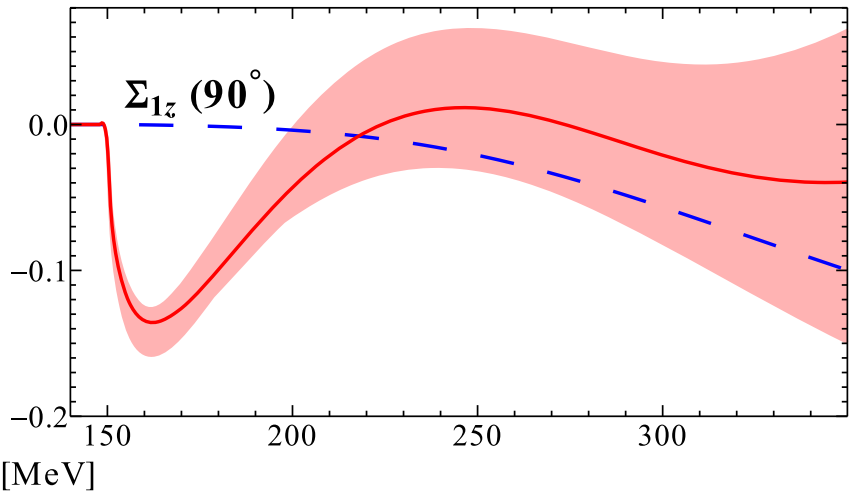

photon energy at $\theta_{\mathrm{cm}}=90^{\circ}$. The theoretical bands correspond to the full calculation and their width is determined as explained in the text. The blue dashed lines correspond to only the Born + Delta-pole graphs included in the calculation

\section{Conclusion}

We have considered low-energy Compton scattering off the nucleon in the framework of manifestly Lorentz-invariant $\chi \mathrm{PT}$, extending our previous calculation [13] to the Deltaresonance region. We have examined the $\mathrm{B} \chi \mathrm{PT}$ predictions for the static and dynamical polarisabilities of the nucleon and resolved their matching in the low-energy expansion.

These predictions are compared with models based on fixed- $t$ dispersion relations (DRs) and the state-of-art heavybaryon $\chi$ PT results. Our results show both similarities and differences to each. Without any exceptions we agree with $\mathrm{HB} \chi \mathrm{PT}$ within the combined errors. The agreement for the scalar dipole polarisabilities (which are fit in $\mathrm{HB} \chi \mathrm{PT}$ but predicted here) is extremely good; however, the central values 
of the spin polarisabilities can be quite different. With DR it is harder to judge as the values do not have systematic uncertainties, but it seems that there is a genuine disagreement in the scalar quadrupole polarisabilities, and probably also in the mixed spin polarisabilities of the neutron. There is also an apparent discrepancy in $\beta_{M 1}$. However, it must be noted that this is not a pure prediction in DR, but relies on rather old fits to Compton data which are not as systematic as the recent chiral ones.

These discrepancies are very intriguing since all of these calculations provide a comparably good description of the Compton scattering observables at energies up to $400 \mathrm{MeV}$. Our results for the polarised observables - beam asymmetry $\Sigma_{3}$ and beam-target asymmetries $\Sigma_{2 x}$ - compare well with the experimental data from LEGS and with the recent results fromA2@MAMI.

The results for $\Sigma_{2 z}$ at zero scattering angle are in remarkable agreement with the recent empirical evaluation based on dispersive sum rules; see Fig. 16. This excellent agreement is due only to the pion-nucleon and pion-Delta loop contributions, thus demonstrating the importance of chiral dynamics in this observable.

Even greater dependence on the chiral dynamics is seen in $\Sigma_{1 z}$ (analogous to the $G$ asymmetry in pion photoproduction); see Fig. 18. In the range between 150 and $200 \mathrm{MeV}$ the dominant contribution to this observable is seen to come from the chiral loops. The $\Sigma_{1 z}$ asymmetry thus seems to be ideally suited for testing chiral dynamics in Compton scattering, and the various approaches are expected to differ here significantly. Any sufficiently precise measurement of this asymmetry is potentially very interesting.

In general, though as we are satisfied to see the predictions of $\mathrm{B} \chi \mathrm{PT}$ to agree with the available data on proton Compton scattering, the range of predictions within the state-of-the-art approaches (including DRs and $\mathrm{HB} \chi \mathrm{PT}$ ) is troublesome. We shall keep looking for opportunities to put these approaches to further experimental tests. Given the ongoing experimental programs at the HIGS and MAMI facilities, we are hopeful that these issues will be resolved in the near future.

Acknowledgments We thank Barbara Pasquini and Aleksandrs Aleksejevs for providing us with the results of their calculations, and Philippe Martel for sending us the recent experimental data on $\Sigma_{2 x}$. V. L. thanks Anatoly L'vov for stimulating discussions. This work was supported by the Deutsche Forschungsgemeinschaft (DFG) through the Collaborative Research Center "The Low-Energy Frontier of the Standard Model" (SFB 1044), by the Russian Federation government Grant No. NSh3830.2014.2, by the MEPhI Academic Excellence Project (Contract No. 02.a03.21.0005), and the UK Science and Technology Facilities Council under Grants ST/J000159/1 and ST/L005794/1.

Open Access This article is distributed under the terms of the Creative Commons Attribution 4.0 International License (http://creativecomm ons.org/licenses/by/4.0/), which permits unrestricted use, distribution, and reproduction in any medium, provided you give appropriate credit to the original author(s) and the source, provide a link to the Creative Commons license, and indicate if changes were made.

Funded by $\mathrm{SCOAP}^{3}$.

\section{Appendix A: Transformation between the bases}

Here, we provide the matrices that transform between several different sets of amplitudes introduced in Sect. 3. In order to transform between any two of these sets, it is enough to know the elements of a chain of transformations that, combined, can connect the two given sets. Here we show some of the minimal set of transformations that result in especially compact expressions, namely, those that transform from $\mathcal{A}_{i}$ to $T_{i}$ and to $A_{i}^{\mathrm{L}}$ (as defined in Ref. [34]):

$$
C^{\mathcal{A} \rightarrow T}=\frac{M_{\mathrm{N}}}{2 v^{2}}\left(\begin{array}{cccccc}
-\frac{\eta-2 v^{2}}{M_{\mathrm{N}}} & 0 & \frac{2 v^{2}}{M_{\mathrm{N}}} & 0 & 0 & 0 \\
-\frac{\eta t^{2}}{2 M_{\mathrm{N}}} & 0 & 0 & 0 & 0 & 0 \\
4 v & \frac{t-4 M_{\mathrm{N}}^{2}}{M_{\mathrm{N}}^{2}} & 0 & 0 & \frac{v\left(4 M^{2}-t\right)}{2 M_{\mathrm{N}}^{2}} & \frac{2 v}{M_{\mathrm{N}}} \\
2 v\left(\eta-2 v^{2}\right) & -2\left(\eta-2 v^{2}\right) & -4 v^{3} & 4 v^{2} & 0 & 0 \\
2 v(\eta+t) & -4\left(\eta-2 v^{2}\right) & 0 & 0 & \eta v & \frac{v t}{M_{\mathrm{N}}} \\
2 v(\eta-t) & -8 v^{2} & 0 & 0 & \eta v & -\frac{v t}{M_{\mathrm{N}}} \\
-\eta v t & \eta t & 0 & 0 & 0 & 0 \\
0 & 0 & 0 & 0 & -\frac{v^{2} t}{M_{\mathrm{N}}} & 0
\end{array}\right)^{\top}
$$




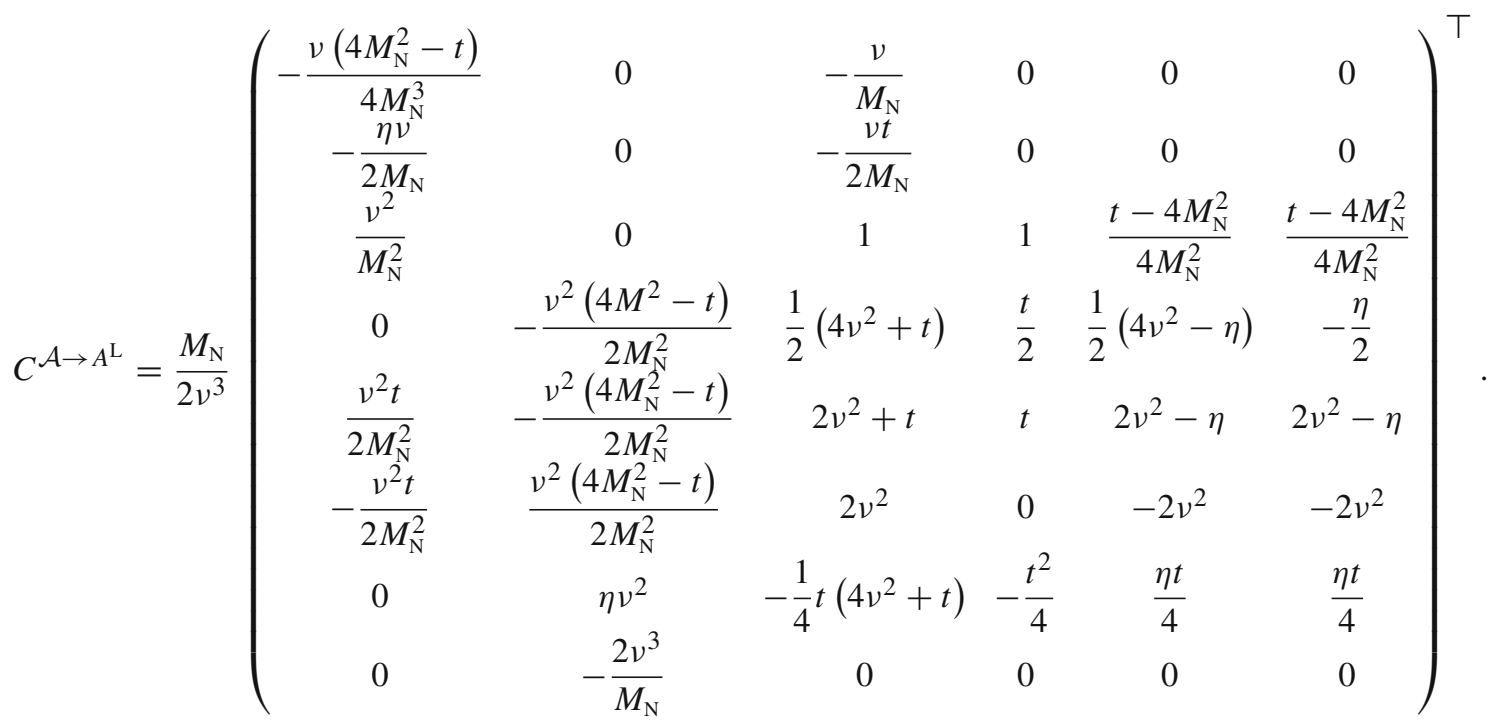

In these equations the kinematical invariants $v$ and $\eta$ are

$v=\frac{s-u}{4 M_{\mathrm{N}}}, \quad \eta=\frac{M_{\mathrm{N}}^{4}-s u}{M_{\mathrm{N}}^{2}}$.

For completeness, we also give the transformation matrix between $T_{i}$ and $A_{i}^{\mathrm{L}}$ [34]:

$C^{T \rightarrow A^{\mathrm{L}}}=\left(\begin{array}{cccccc}\frac{1}{t} & \frac{v}{t} & \frac{1}{t} & \frac{v}{t} & 0 & 0 \\ 0 & \frac{v}{t} & 0 & \frac{v}{t} & \frac{2}{t} & 0 \\ \frac{1}{\eta} & -\frac{t}{4 \eta v} & -\frac{1}{\eta} & \frac{t}{4 \eta v} & 0 & 0 \\ 0 & -\frac{t}{4 \eta v} & 0 & \frac{t}{4 \eta v} & 0 & \frac{2 M}{\eta} \\ 0 & \frac{1}{4 v} & 0 & \frac{1}{4 v} & 0 & 0 \\ 0 & \frac{1}{4 v} & 0 & -\frac{1}{4 v} & 0 & 0\end{array}\right)$

$$
C^{T \rightarrow A^{\mathrm{cm}}}=\frac{1}{2 M_{\mathrm{N}}}\left(\begin{array}{cccccc}
0 & 0 & \frac{\left(W_{+}\right)^{2}-z\left(W_{-}\right)^{2}}{2 W} & \omega\left(z W_{-}+W_{+}\right) & 0 & 0 \\
\frac{\left(W_{+}\right)^{2}-z\left(W_{-}\right)^{2}}{2 W\left(1-z^{2}\right)} & \frac{\omega\left(z W_{-}+W_{+}\right)}{1-z^{2}} & \frac{z\left(W_{+}\right)^{2}-z^{2}\left(W_{-}\right)^{2}}{2 W\left(1-z^{2}\right.} & \frac{\omega z\left(z W_{-}+W_{+}\right)}{1-z^{2}} & 0 & 0 \\
\omega-W_{-} & \omega W_{-} & z\left(\omega-W_{-}\right) & \omega z W_{-} & 2 \omega & 2 \omega W \\
0 & 0 & \omega-W_{-} & \omega W_{-} & 0 & 0 \\
\frac{z\left(W_{-}-\omega\right)}{z^{2}-1} & \frac{\omega z W_{-}}{1-z^{2}} & \frac{z^{2}\left(W_{-}-\omega\right)}{z^{2}-1} & \frac{\omega z^{2} W_{-}}{1-z^{2}} & \frac{\omega}{1-z} & -\frac{\omega W}{z+1} \\
\frac{\omega-W_{-}}{z^{2}-1} & \frac{\omega W_{-}}{z^{2}-1} & \frac{z\left(\omega-W_{-}\right)}{z^{2}-1} & \frac{\omega z W_{-}}{z^{2}-1} & \frac{\omega}{z-1} & -\frac{\omega W}{z+1}
\end{array}\right),
$$

where $W=\sqrt{s}, W_{ \pm}=W \pm M_{\mathrm{N}}$, and $\omega=\left(W^{2}-M_{\mathrm{N}}^{2}\right) /(2 W)$ and $z=\cos \theta_{\mathrm{cm}}$ refer to the $\mathrm{cm}$-frame photon energy and scattering angle.

The transformation between $A_{i}$ and $A^{\mathrm{Br}}$ is given in the appendix of Ref. [11].

Finally, the transformation matrix between $A_{i}^{\mathrm{L}}$ and the Breit-frame amplitudes $A_{i}^{\mathrm{Br}}$ is 


$$
C^{A^{\mathrm{L}} \rightarrow A^{\mathrm{Br}}}=\frac{E_{\mathrm{N}} \omega_{B}^{2}}{M_{\mathrm{N}}}\left(\begin{array}{cccccc}
(z-1) & 0 & -\frac{E_{\mathrm{N}}^{2}(z+1)}{M_{\mathrm{N}}^{2}} & 0 & \frac{\omega_{B}^{2}(z-1)}{M_{\mathrm{N}}^{2}} & -(z+1) \\
-1 & 0 & \frac{E_{\mathrm{N}}^{2}}{M_{\mathrm{N}}^{2}} & 0 & -\frac{\omega_{B}^{2}}{M_{\mathrm{N}}^{2}} & 1 \\
0 & \frac{\omega_{B}(z-1)}{E_{\mathrm{N}}} & 0 & \frac{E_{\mathrm{N}}^{2} \omega_{B}(z+1)}{M_{\mathrm{N}}^{3}} & \frac{\omega_{B}\left(M_{\mathrm{N}}(z+1)-2 E_{\mathrm{N}}\right)}{M_{\mathrm{N}}^{2}} & 0 \\
0 & 0 & 0 & 0 & \frac{\omega_{B}}{M_{\mathrm{N}}} & -\frac{\omega_{B}}{M_{\mathrm{N}}} \\
0 & -\frac{\omega_{B}}{2 E_{\mathrm{N}}} & 0 & -\frac{E_{\mathrm{N}}^{2} \omega_{B}}{2 M_{\mathrm{N}}^{3}} & \frac{\omega_{B}\left(E_{\mathrm{N}}-M_{\mathrm{N}} z\right)}{M_{\mathrm{N}}^{2}(z-1)} & \frac{\omega_{B}}{2 M_{\mathrm{N}}} \\
0 & \frac{\omega_{B}}{2 E_{\mathrm{N}}} & 0 & -\frac{E_{\mathrm{N}}^{2} \omega_{B}}{2 M_{\mathrm{N}}^{3}} & \frac{\omega_{B}\left(M_{\mathrm{N}}-E_{\mathrm{N}}\right)}{M_{\mathrm{N}}^{2}(z-1)} & -\frac{\omega_{B}}{2 M_{\mathrm{N}}}
\end{array}\right),
$$

where $\omega_{B}$ and $z=\cos \theta_{B}$ here refer to the Breit-frame photon energy and scattering angle, and $E_{\mathrm{N}}$ is the nucleon energy (equal before and after the collision), with $E_{\mathrm{N}}=v M_{\mathrm{N}} / \omega_{B}=$ $\sqrt{M_{\mathrm{N}}^{2}-t / 4}$.

Note that the amplitudes $\mathcal{A}_{i}, A_{i}^{\mathrm{L}}$ and $T_{i}$ are defined assuming a covariant spinor normalisation of $2 M_{\mathrm{N}}$, whereas the amplitudes in the non-relativistic bases, $A_{i}^{\mathrm{Br}}$ and $A_{i}^{\mathrm{cm}}$, assume a Pauli spinor normalisation of unity.

\section{Appendix B: Matching of higher-order polarisabilities}

Here we provide the relations between the polarisabilities, static and dynamic, and the L'vov amplitudes of Ref. [34]. The latter can be expanded in the crossing-invariant variables $v=(s-u) /\left(2 M_{\mathrm{N}}\right)$ and $t$ :

$A_{i}^{\mathrm{L}}(\nu, t)=a_{i}+a_{i, \nu} \nu^{2}+a_{i, t} t+\cdots$.

The non-Born part of the Taylor coefficients $a_{i}, a_{i, \nu}, a_{i, t}$ can be related to polarisabilities as follows.

We have already argued that crossing symmetry means that static polarisabilities should be defined in the Breit frame, so using $A_{i}^{\mathrm{Br}}=\sum_{j} C_{i j}^{\mathrm{A}^{\mathrm{L}} \rightarrow A^{\mathrm{Br}}} A_{i}^{\mathrm{L}}$ with the transformation matrix defined in Eq. (A6) gives, for example,

$$
\begin{aligned}
A_{1}^{\mathrm{Br}}= & \frac{E_{\mathrm{N}}}{M_{\mathrm{N}}}\left(\left(a_{1}-a_{3}-a_{6}\right) z-a_{1}-a_{3}-a_{6}\right) \omega_{\mathrm{B}}^{2} \\
& +\left(-a_{1, v}-a_{3, v}-a_{6, v}+2\left(a_{1, t}+a_{3, t}+a_{6, t}\right)\right. \\
& +z\left(a_{1, v}-a_{3, v}-a_{6, v}-4 a_{1, t}\right) \\
& +2 z^{2}\left(a_{1, t}-a_{3, t}-a_{6, t}\right) \\
& \left.+\frac{1}{2 M_{\mathrm{N}}^{2}}\left(a_{3} z^{2}+2 a_{5} z-a_{3}-2 a_{5}\right)\right) \omega_{\mathrm{B}}^{4}+\mathcal{O}\left(\omega_{\mathrm{B}}^{6}\right) .
\end{aligned}
$$

where $\omega_{\mathrm{B}}$ and $z=\cos \theta_{\mathrm{B}}$ here refer to the Breit-frame photon energy and scattering angle.
By comparing these to the direct results of the effective Hamiltonians Eqs. (11-14) as given in Eq. (16), we obtain

$$
\begin{aligned}
& 4 \pi \alpha_{E 1}=-a_{1}-a_{3}-a_{6}, \quad 4 \pi \beta_{M 1}=a_{1}-a_{3}-a_{6}, \\
& 4 \pi \gamma_{E 1 E 1}= \frac{a_{2}-a_{4}+2 a_{5}+a_{6}}{2 M_{\mathrm{N}}}, \\
& 4 \pi \gamma_{M 1 M 1}= \frac{-a_{2}-a_{4}-2 a_{5}+a_{6}}{2 M_{\mathrm{N}}}, \\
& 4 \pi \gamma_{M 1 E 2}= \frac{-a_{2}-a_{4}-a_{6}}{2 M_{\mathrm{N}}}, \\
& 4 \pi \gamma_{E 1 M 2}= \frac{a_{2}-a_{4}-a_{6}}{2 M_{\mathrm{N}}}, \\
& 4 \pi \alpha_{E 2}=-12\left(a_{1, t}+a_{3, t}+a_{6, t}\right)+\frac{3 a_{3}}{M_{\mathrm{N}}^{2}}, \\
& 4 \pi \beta_{M 2}=12\left(a_{1, t}-a_{3, t}-a_{6, t}\right)+\frac{3 a_{3}}{M_{\mathrm{N}}^{2}}, \\
& 4 \pi \alpha_{E 1, v}= 3 a_{1, t}-a_{1, v}+a_{3, t}-a_{3, v}+a_{6, t}-a_{6, v} \\
&+\frac{-a_{3}-4 a_{5}}{4 M_{\mathrm{N}}^{2}}, \\
& 4 \pi \beta_{M 1, v}=-3 a_{1, t}+a_{1, v}+a_{3, t}-a_{3, v}+a_{6, t}-a_{6, v} \\
&+\frac{4 a_{5}-a_{3}}{4 M_{\mathrm{N}}^{2}},
\end{aligned}
$$

$4 \pi \gamma_{E 2 E 2}=+\frac{a_{2, t}-a_{4, t}+3 a_{5, t}+2 a_{6, t}}{6 M_{\mathrm{N}}}+\frac{a_{2}+2 a_{4}}{48 M_{\mathrm{N}}^{3}}$,

$4 \pi \gamma_{M 2 M 2}=\frac{-a_{2, t}-a_{4, t}-3 a_{5, t}+2 a_{6, t}}{6 M_{\mathrm{N}}}+\frac{2 a_{4}-a_{2}}{48 M_{\mathrm{N}}^{3}}$,

$4 \pi \gamma_{M 2 E 3}=\frac{-a_{2, t}-a_{4, t}-a_{6, t}}{3 M_{\mathrm{N}}}+\frac{2 a_{4}-a_{2}}{24 M_{\mathrm{N}}^{3}}$,

$4 \pi \gamma_{E 2 M 3}=\frac{a_{2, t}-a_{4, t}-a_{6, t}}{3 M_{\mathrm{N}}}+\frac{a_{2}+2 a_{4}}{24 M_{\mathrm{N}}^{3}}$,

$$
\begin{aligned}
4 \pi \gamma_{E} 1 E 1, v \\
=\frac{-3 a_{2, t}+a_{2, v}+a_{4, t}-a_{4, v}-5 a_{5, t}+2 a_{5, v}-2 a_{6, t}+a_{6, v}}{2 M_{\mathrm{N}}} \\
\quad+\frac{-3 a_{2}-2\left(a_{4}-2 a_{5}\right)}{16 M_{\mathrm{N}}^{3}}
\end{aligned}
$$




$$
\begin{aligned}
4 \pi & \gamma_{M 1 M 1, v} \\
= & \frac{3 a_{2, t}-a_{2, v}+a_{4, t}-a_{4, v}+5 a_{5, t}-2 a_{5, v}-2 a_{6, t}+a_{6, v}}{2 M_{\mathrm{N}}} \\
& \quad+\frac{3 a_{2}-2\left(a_{4}+2 a_{5}\right)}{16 M_{\mathrm{N}}^{3}}, \\
4 \pi \gamma_{M 1 E 2, v} & \\
= & \frac{18 a_{2, t}-5 a_{2, v}+2 a_{4, t}-5 a_{4, v}+10 a_{5, t}+12 a_{6, t}-5 a_{6, v}}{10 M_{\mathrm{N}}} \\
& \quad+\frac{9 a_{2}-2\left(a_{4}+5 a_{5}\right)}{40 M_{\mathrm{N}}^{3}}, \\
4 \pi \gamma_{E} 1 M 2, v & \frac{-18 a_{2, t}+5 a_{2, v}+2 a_{4, t}-5 a_{4, v}-10 a_{5, t}+12 a_{6, t}-5 a_{6, v}}{10 M_{\mathrm{N}}} \\
\quad & \quad \frac{-9 a_{2}-2\left(a_{4}-5 a_{5}\right)}{40 M_{\mathrm{N}}^{3}} .
\end{aligned}
$$

The first seven lines of this, namely the dipole polarisabilities and the spin-independent quadrupole polarisabilities, agree with Babusci et al. [34]. The remaining terms, the spin-dependent quadrupole polarisabilities, were considered by Holstein et al. [23]. However, those authors, while quoting the earlier paper for the spin-independent quadrupoles, proceed in the centre-of-mass frame, and so the matching is not consistent. It should also be noted that Holstein et al. projected their polarisabilities directly from the c.m. amplitudes
$A_{i}^{\mathrm{cm}}$ as defined in Eq. (9), and not, as is usual for the dynamical polarisabilities, from the Ragusa amplitudes which are related to the former by a factor of $\sqrt{s} / M_{\mathrm{N}}$.

It should be noted that with the definitions above, the definition of $\bar{\gamma}_{0}$ in terms of quadrupole polarisabilities in Eq. (18) is exact and not subject to recoil corrections, in contradistinction to that of Ref. [35].

In order to match to the dynamical polarisabilities defined in the c.m. frame, we plug the same Taylor expansion of the L'vov amplitudes Eq. (B1) into the expressions given in appendix A of Ref. [38] for the helicity amplitudes and partial waves $f_{E E}^{l \pm}$ etc., then use the definitions of the dynamical polarisabilities of Eqs. (23-25) above, to get for example

$$
\begin{aligned}
& 4 \pi \alpha_{E 1}(\omega)=-\left(a_{1}+a_{3}+a_{6}\right)+\left(a_{1}-a_{3}-a_{6}\right) \frac{\omega}{M_{\mathrm{N}}} \\
& -\left(7 a_{1}+5 a_{3}+8 a_{5}+3 a_{6}+8 M_{\mathrm{N}}^{2} \times\left(a_{1, v}-3 a_{1, t}\right.\right. \\
& \left.\left.+a_{3, v}-a_{3, t}+a_{6, v}-a_{6, t}\right)\right) \frac{\omega^{2}}{8 M_{\mathrm{N}}^{2}}+\cdots .
\end{aligned}
$$

Equation (B3) can be inverted to give the $a_{i}$ in terms of the static polarisabilities, and on substituting into Eq. (B4) it gives

$$
\begin{aligned}
& \alpha_{E 1}(\omega)=\alpha_{E 1}+\frac{\omega \beta_{M 1}}{M_{\mathrm{N}}}+\omega^{2}\left(\alpha_{E 1, v}+\frac{5 \alpha_{E 1}-2 \beta_{M 1}}{8 M_{\mathrm{N}}^{2}}\right)+\cdots, \\
& \beta_{M 1}(\omega)=\beta_{M 1}+\frac{\omega \alpha_{E 1}}{M_{\mathrm{N}}}+\omega^{2}\left(\beta_{M 1, v}-\frac{2 \alpha_{E 1}-5 \beta_{M 1}}{8 M_{\mathrm{N}}^{2}}\right)+\cdots, \\
& \gamma_{E 1 E 1}(\omega)=\gamma_{E 1 E 1}+\omega\left(\frac{4 \gamma_{E 1 E 1}+7 \gamma_{M 1 E 2}+5 \gamma_{M 1 M 1}}{8 M_{\mathrm{N}}}+\frac{\beta_{M 1}}{16 M_{\mathrm{N}}^{2}}\right) \\
& +\omega^{2}\left(\gamma_{E 1 E 1, v}+\frac{12 \gamma_{E 1 E 1}+9 \gamma_{E 1 M 2}+4 \gamma_{M 1 E 2}+3 \gamma_{M 1 M 1}}{16 M_{\mathrm{N}}^{2}}+\frac{\alpha_{E 1}}{16 M_{\mathrm{N}}^{3}}\right)+\cdots, \\
& \gamma_{M 1 M 1}(\omega)=\gamma_{M 1 M 1}+\omega\left(\frac{5 \gamma_{E 1 E 1}+7 \gamma_{E 1 M 2}+4 \gamma_{M 1 M 1}}{8 M_{\mathrm{N}}}+\frac{\alpha_{E 1}}{16 M_{\mathrm{N}}^{2}}\right) \\
& +\omega^{2}\left(\gamma_{M 1 M 1, v}+\frac{3 \gamma_{E 1 E 1}+4 \gamma_{E 1 M 2}+9 \gamma_{M 1 E 2}+12 \gamma_{M 1 M 1}}{16 M_{\mathrm{N}}^{2}}+\frac{\beta_{M 1}}{16 M_{\mathrm{N}}^{3}}\right)+\cdots, \\
& \gamma_{E 1 M 2}(\omega)=\gamma_{E 1 M 2}+\omega\left(\frac{2 \gamma_{E 1 M 2}+3\left(\gamma_{M 1 E 2}+\gamma_{M 1 M 1}\right)}{4 M_{\mathrm{N}}}-\frac{\beta_{M 1}}{8 M_{\mathrm{N}}^{2}}\right) \\
& +\omega^{2}\left(\gamma_{E 1 M 2, v}-\frac{\alpha_{E 1}}{8 M_{\mathrm{N}}^{3}}+\frac{23 \gamma_{E 1 E 1}+34 \gamma_{E 1 M 2}+9 \gamma_{M 1 E 2}+10 \gamma_{M 1 M 1}}{40 M_{\mathrm{N}}^{2}}\right)+\cdots, \\
& \gamma_{M 1 E 2}(\omega)=\gamma_{M 1 E 2}+\omega\left(\frac{3 \gamma_{E 1 E 1}+3 \gamma_{E 1 M 2}+2 \gamma_{M 1 E 2}}{4 M_{\mathrm{N}}}-\frac{\alpha_{E 1}}{8 M_{\mathrm{N}}^{2}}\right) \\
& +\omega^{2}\left(\gamma_{M 1 E 2, v}+\frac{10 \gamma_{E 1 E 1}+9 \gamma_{E 1 M 2}+34 \gamma_{M 1 E 2}+23 \gamma_{M 1 M 1}}{40 M_{\mathrm{N}}^{2}}-\frac{\beta_{M 1}}{8 M_{\mathrm{N}}^{3}}\right)+\cdots
\end{aligned}
$$


and

$$
\begin{aligned}
\alpha_{E 2}(\omega)=\alpha_{E 2}+ & \frac{3 \beta_{M 1}}{2 M_{\mathrm{N}}^{2}}+\omega\left(\frac{12 \alpha_{E 1, \nu}+2 \alpha_{E 2}+\beta_{M 2}}{2 M_{\mathrm{N}}}\right. \\
& \left.+\frac{3 \alpha_{E 1}}{2 M_{\mathrm{N}}^{3}}+\frac{3\left(\gamma_{E 1 E 1}-\gamma_{E 1 M 2}\right)}{2 M_{\mathrm{N}}^{2}}\right)+\cdots, \\
\beta_{M 2}(\omega)= & \beta_{M 2}+\frac{3 \alpha_{E 1}}{2 M_{\mathrm{N}}^{2}}+\omega\left(\frac{12 \beta_{M 1, v}+\alpha_{E 2}+2 \beta_{M 2}}{2 M_{\mathrm{N}}}\right. \\
+ & \left.\frac{3 \beta_{M 1}}{2 M_{\mathrm{N}}^{3}}-\frac{3\left(\gamma_{M 1 E 2}-\gamma_{M 1 M 1}\right)}{2 M_{\mathrm{N}}^{2}}\right)+\cdots, \\
\gamma_{E 2 E 2}(\omega)= & \frac{1}{\omega}\left(\frac{\alpha_{E 1}}{16 M_{\mathrm{N}}^{2}}+\frac{\gamma_{E 1 E 1}-\gamma_{E 1 M 2}}{8 M_{\mathrm{N}}}\right)+\gamma_{E 2 E 2} \\
& +\frac{3 \gamma_{E 1 E 1}-2 \gamma_{E 1 M 2}+\gamma_{M 1 E 2}+2 \gamma_{M 1 M 1}}{48 M_{\mathrm{N}}^{2}} \\
& +\frac{\beta_{M 1}}{16 M_{\mathrm{N}}^{3}}+\cdots, \\
& \frac{1}{\omega}\left(\frac{\beta_{M 1}}{16 M_{\mathrm{N}}^{2}}-\frac{\gamma_{M 1 E 2}-\gamma_{M 1 M 1}}{8 M_{\mathrm{N}}}\right)+\gamma_{M 2 M 2} \\
& +\frac{2 \gamma_{E 1 E 1}+\gamma_{E 1 M 2}-2 \gamma_{M 1 E 2}+3 \gamma_{M 1 M 1}}{48 M_{\mathrm{N}}^{2}} \\
& +\frac{\alpha_{E 1}}{16 M_{\mathrm{N}}^{3}}+\cdots,
\end{aligned}
$$

$\gamma_{E 2 M 3}(\omega)=\gamma_{E 2 M 3}+\frac{\gamma_{E 1 M 2}+\gamma_{M 1 E 2}+2 \gamma_{M 1 M 1}}{24 M_{\mathrm{N}}^{2}}+\cdots$,

$\gamma_{M 2 E 3}(\omega)=\gamma_{M 2 E 3}+\frac{2 \gamma_{E 1 E 1}+\gamma_{E 1 M 2}+\gamma_{M 1 E 2}}{24 M_{\mathrm{N}}^{2}}+\cdots$.

Note the divergent $1 / \omega$ pieces in the unmixed spin quadrupole polarisabilities, generated by the recoil terms. As noted above, there are no recoil corrections to the $l=1$ polarisabilities at leading order in $\omega$.

To conclude this appendix, we provide here the numerical values of the non-Born coefficients $a_{i}, a_{i, v}$ and $a_{i, t}$ that result from our calculation; see Table 7. Our calculations have been performed with the use of computational packages FORM [63] and LoopTools [64].

\section{Appendix C: Expressions for static polarisabilities}

Here we collect the analytic expressions for the various con-

\begin{tabular}{|c|c|c|c|c|c|c|}
\hline \multicolumn{3}{|c|}{$\pi \mathrm{N}$ loop } & \multirow[t]{2}{*}{$\pi \Delta$ loop } & \multirow[t]{2}{*}{$\Delta$ pole } & \multicolumn{2}{|l|}{ Total } \\
\hline & Proton & Neutron & & & Proton & Neutron \\
\hline$a_{1}$ & -55.06 & -65.86 & -36.89 & 45.61 & -46.34 & -57.14 \\
\hline$a_{2}$ & -22.70 & -44.75 & 13.42 & -48.57 & -57.85 & -79.9 \\
\hline$a_{3}$ & 116.35 & 151.98 & -28.29 & -131.71 & -43.65 & -8.03 \\
\hline$a_{4}$ & 60.15 & 88.92 & -3.05 & -84.98 & -27.88 & 0.89 \\
\hline$a_{5}$ & -85.73 & -114.79 & 12.93 & -85.82 & -158.62 & -187.68 \\
\hline$a_{6}$ & -148.38 & -204.00 & 9.58 & 87.48 & -51.33 & -106.94 \\
\hline$a_{1, v}$ & -24.16 & -30.09 & -2.25 & 34.76 & 8.35 & 2.42 \\
\hline$a_{2, v}$ & -71.01 & -82.86 & 2.59 & -20.03 & -88.45 & -100.31 \\
\hline$a_{3, v}$ & 92.46 & 121.44 & -1.43 & -44.44 & 46.58 & 75.56 \\
\hline$a_{4, v}$ & 62.28 & 87.10 & -0.28 & -28.67 & 33.32 & 58.15 \\
\hline$a_{5, v}$ & -79.57 & -105.54 & 0.59 & -28.96 & -107.94 & -133.91 \\
\hline$a_{6, v}$ & -111.01 & -148.18 & 0.65 & 26.08 & -84.28 & -121.46 \\
\hline$a_{1, t}$ & -11.49 & -11.07 & -3.04 & -2.65 & -17.18 & -16.76 \\
\hline$a_{2, t}$ & 0.94 & 2.02 & -0.14 & 2.31 & 3.10 & 4.18 \\
\hline$a_{3, t}$ & 5.15 & 1.68 & -1.13 & 7.01 & 11.03 & 7.56 \\
\hline$a_{4, t}$ & -1.83 & -4.81 & 0.08 & 5.58 & 3.84 & 0.85 \\
\hline$a_{5, t}$ & -5.15 & -2.23 & 0.27 & 6.60 & 1.71 & 4.63 \\
\hline$a_{6, t}$ & -6.55 & -1.95 & 0.55 & -6.43 & -12.43 & -7.82 \\
\hline
\end{tabular}
tributions computed in this work. The corresponding numerical results are shown in Tables 3, 4, 5 and 6. The spin polarisabilities $\gamma_{1}-\gamma_{4}$ provided here are related to those defined in Sect. 4.1 by

$\gamma_{E 1 E 1}=-\gamma_{1}-\gamma_{3}, \quad \gamma_{M 1 M 1}=\gamma_{4}, \quad \gamma_{E 1 M 2}=\gamma_{3}$,

$$
\gamma_{M 1 E 2}=\gamma_{2}+\gamma_{4}
$$

Table 7 Predictions for the parameters of the Taylor expansion of the L'vov amplitudes $A_{i}^{\mathrm{L}}(v, t)$, in units of $10^{4} \mathrm{fm}^{-3}$ for $a_{i}$ and $10^{4} \mathrm{fm}^{-5}$ for $a_{i, t}$ and $a_{i, v}$

\section{$1 \pi \mathrm{N}$ loops}

\section{a Proton}

The complete $O\left(p^{3}\right)$ chiral-loop contribution to the dipole, spin and quadrupole polarisabilities of the proton are (with $\left.\mu=m_{\pi} / M_{\mathrm{N}}\right)$

$$
\begin{aligned}
\alpha_{E 1}^{(\pi \mathrm{N})}= & \frac{e^{2} g_{A}^{2}}{192 \pi^{3} f_{\pi}^{2} M_{\mathrm{N}}} \frac{1}{\mu\left(4-\mu^{2}\right)^{5 / 2}} \\
& \times\left[\mu\left(-18 \mu^{6}+157 \mu^{4}-407 \mu^{2}+304\right) \sqrt{4-\mu^{2}}\right. \\
& +\mu\left(9 \mu^{4}-20 \mu^{2}+9\right)\left(4-\mu^{2}\right)^{5 / 2} \log \mu^{2} \\
& -\left(9 \mu^{10}-110 \mu^{8}+479 \mu^{6}-870 \mu^{4}\right. \\
& \left.\left.+590 \mu^{2}-80\right) \arccos \left(\frac{\mu^{2}}{2}-1\right)\right],
\end{aligned}
$$

$$
\begin{aligned}
\beta_{M 1}^{(\pi \mathrm{N})}= & -\frac{e^{2} g_{A}^{2}}{192 \pi^{3} f_{\pi}^{2} M_{\mathrm{N}}} \frac{1}{\mu\left(4-\mu^{2}\right)^{3 / 2}} \\
& \times\left[\mu\left(-54 \mu^{4}+235 \mu^{2}-127\right) \sqrt{4-\mu^{2}}\right. \\
& -\mu\left(27 \mu^{4}-50 \mu^{2}+9\right)\left(4-\mu^{2}\right)^{3 / 2} \log \mu^{2}
\end{aligned}
$$




$$
\begin{aligned}
& -\left(27 \mu^{8}-212 \mu^{6}+471 \mu^{4}-246 \mu^{2}+2\right) \\
& \left.\times \arccos \left(\frac{\mu^{2}}{2}-1\right)\right], \\
\gamma_{1}^{(\pi \mathrm{N})}= & \frac{e^{2} g_{A}^{2}}{192 \pi^{3} f_{\pi}^{2} M_{\mathrm{N}}^{2}} \frac{1}{\mu^{2}\left(4-\mu^{2}\right)^{5 / 2}} \\
& \times\left[\sqrt { 4 - \mu ^ { 2 } } \left(18 \mu^{8}-173 \mu^{6}+524 \mu^{4}-452 \mu^{2}\right.\right. \\
& \left.-\left(4-\mu^{2}\right)^{2}\left(9 \mu^{4}-28 \mu^{2}+13\right) \mu^{2} \log \mu^{2}+32\right) \\
& +\mu\left(9 \mu^{10}-118 \mu^{8}+563 \mu^{6}-1150 \mu^{4}\right. \\
& \left.\left.+848 \mu^{2}-104\right) \arccos \left(\frac{\mu^{2}}{2}-1\right)\right],
\end{aligned}
$$$$
\gamma_{2}^{(\pi \mathrm{N})}=\frac{e^{2} g_{A}^{2}}{96 \pi^{3} f_{\pi}^{2} M_{\mathrm{N}}^{2}} \frac{1}{\mu^{2}\left(4-\mu^{2}\right)^{5 / 2}}
$$$$
\times\left[2 \sqrt { 4 - \mu ^ { 2 } } \left(18 \mu^{8}-149 \mu^{6}+348 \mu^{4}-179 \mu^{2}\right.\right.
$$$$
\left.-\left(4-\mu^{2}\right)^{2}\left(9 \mu^{4}-16 \mu^{2}+4\right) \mu^{2} \log \mu^{2}+4\right)
$$$$
+\mu\left(18 \mu^{10}-212 \mu^{8}+868 \mu^{6}-1400 \mu^{4}\right.
$$$$
\left.\left.+701 \mu^{2}-44\right) \arccos \left(\frac{\mu^{2}}{2}-1\right)\right] \text {, }
$$$$
\gamma_{3}^{(\pi \mathrm{N})}=-\frac{e^{2} g_{A}^{2}}{384 \pi^{3} f_{\pi}^{2} M_{\mathrm{N}}^{2}} \frac{1}{\mu^{2}\left(4-\mu^{2}\right)^{5 / 2}}
$$$$
\times\left[\sqrt { 4 - \mu ^ { 2 } } \left(18 \mu^{8}-141 \mu^{6}+284 \mu^{4}-4 \mu^{2}\right.\right.
$$$$
\left.-3\left(\mu^{2}-4\right)^{2}\left(3 \mu^{4}-4 \mu^{2}-1\right) \mu^{2} \log \mu^{2}-16\right)
$$$$
+\mu\left(9 \mu^{10}-102 \mu^{8}+387 \mu^{6}-510 \mu^{4}\right.
$$$$
\left.\left.+46 \mu^{2}+56\right) \arccos \left(\frac{\mu^{2}}{2}-1\right)\right] \text {, }
$$

$$
\begin{aligned}
& \gamma_{4}^{(\pi \mathrm{N})}=\frac{e^{2} g_{A}^{2}}{384 \pi^{3} f_{\pi}^{2} M_{\mathrm{N}}^{2}} \frac{1}{\mu^{2}\left(4-\mu^{2}\right)^{5 / 2}} \\
& \times\left[\sqrt { 4 - \mu ^ { 2 } } \left(-126 \mu^{8}+1047 \mu^{6}-2462 \mu^{4}+1308 \mu^{2}\right.\right. \\
& \left.+\left(\mu^{2}-4\right)^{2}\left(63 \mu^{4}-114 \mu^{2}+29\right) \mu^{2} \log \mu^{2}-16\right) \\
& +\left(-63 \mu^{11}+744 \mu^{9}-3059 \mu^{7}+4970 \mu^{5}\right. \\
& \left.\left.-2534 \mu^{3}+152 \mu\right) \arccos \left(\frac{\mu^{2}}{2}-1\right)\right],
\end{aligned}
$$

$$
\begin{aligned}
& \alpha_{E 2}^{(\pi \mathrm{N})}=-\frac{3}{2 M_{\mathrm{N}}^{2}} \beta_{M 1}^{(\pi \mathrm{N})}+\frac{e^{2} g_{A}^{2}}{1920 \pi^{3} f_{\pi}^{2} M_{\mathrm{N}}^{3}} \frac{1}{\mu^{3}\left(4-\mu^{2}\right)^{7 / 2}} \\
& \times\left[\mu \sqrt { 4 - \mu ^ { 2 } } \left(2610 \mu^{10}-33,069 \mu^{8}+148,813 \mu^{6}\right.\right. \\
& -273,436 \mu^{4}+168,848 \mu^{2}-14144 \\
& \left.-3\left(\mu^{2}-4\right)^{3}\left(435 \mu^{4}-944 \mu^{2}+385\right) \mu^{2} \log \mu^{2}\right) \\
& +3\left(435 \mu^{14}-7034 \mu^{12}+44,051 \mu^{10}-132,370 \mu^{8}\right. \\
& \left.+189,490 \mu^{6}-107,960 \mu^{4}+12,576 \mu^{2}+896\right) \\
& \left.\quad \times \arccos \left(\frac{\mu^{2}}{2}-1\right)\right],
\end{aligned}
$$

$$
\begin{aligned}
& \beta_{M 2}^{(\pi \mathrm{N})}=-\frac{3}{2 M_{\mathrm{N}}^{2}} \alpha_{E 1}^{(\pi \mathrm{N})}+\frac{e^{2} g_{A}^{2}}{1920 \pi^{3} f_{\pi}^{2} M_{\mathrm{N}}^{3}} \frac{1}{\mu^{3}\left(4-\mu^{2}\right)^{5 / 2}} \\
& \times\left[\mu \sqrt { 4 - \mu ^ { 2 } } \left(-4230 \mu^{8}+35,619 \mu^{6}-86,105 \mu^{4}\right.\right. \\
& +49,108 \mu^{2}-368+3\left(\mu^{2}-4\right)^{2} \\
& \left.\times\left(705 \mu^{4}-1354 \mu^{2}+385\right) \mu^{2} \log \mu^{2}\right) \\
& -3\left(705 \mu^{12}-8404 \mu^{10}+35,075 \mu^{8}-58,570 \mu^{6}\right. \\
& \left.\left.\quad+31,630 \mu^{4}-2200 \mu^{2}+96\right) \arccos \left(\frac{\mu^{2}}{2}-1\right)\right] .
\end{aligned}
$$

\section{$b$ Neutron}

The complete $O\left(p^{3}\right)$ chiral-loop contribution to the dipole, spin and quadrupole polarisabilities of the neutron are (with $\left.\mu=m_{\pi} / M_{\mathrm{N}}\right)$

$$
\begin{aligned}
& \alpha_{E 1}^{(\pi \mathrm{N}, n)}=\frac{e^{2} g_{A}^{2}}{192 \pi^{3} f_{\pi}^{2} M_{N}} \frac{1}{\mu\left(4-\mu^{2}\right)^{5 / 2}} \\
& \quad \times\left[\mu \sqrt{4-\mu^{2}}\left(-\mu^{2}+3\left(\mu^{2}-4\right)^{2} \log \mu^{2}+16\right)\right. \\
& \left.\quad+\left(-3 \mu^{6}+30 \mu^{4}-98 \mu^{2}+80\right) \arccos \left(\frac{\mu^{2}}{2}-1\right)\right],
\end{aligned}
$$

$$
\begin{aligned}
\beta_{M 1}^{(\pi \mathrm{N}, n)}= & -\frac{e^{2} g_{A}^{2}}{192 \pi^{3} f_{\pi}^{2} M_{N}} \frac{1}{\mu\left(4-\mu^{2}\right)^{3 / 2}} \\
& \times\left[\mu \sqrt{4-\mu^{2}}\left(3\left(\mu^{2}-4\right) \log \mu^{2}-11\right)\right. \\
& \left.-\left(3 \mu^{4}-18 \mu^{2}+2\right) \arccos \left(\frac{\mu^{2}}{2}-1\right)\right],
\end{aligned}
$$

(C10) 


$$
\begin{aligned}
\gamma_{1}^{(\pi \mathrm{N}, n)}= & \frac{e^{2} g_{A}^{2}}{96 \pi^{3} f_{\pi}^{2} M_{N}^{2}} \frac{1}{\mu^{2}\left(4-\mu^{2}\right)^{5 / 2}} \\
& \times\left[\sqrt { 4 - \mu ^ { 2 } } \left(2\left(2 \mu^{4}-7 \mu^{2}+8\right)\right.\right. \\
& \left.-\mu^{2}\left(\mu^{2}-4\right)^{2} \log \mu^{2}\right)+\mu\left(\mu^{6}-10 \mu^{4}\right. \\
& \left.\left.+24 \mu^{2}-12\right) \arccos \left(\frac{\mu^{2}}{2}-1\right)\right], \\
\gamma_{2}^{(\pi \mathrm{N}, n)}= & \frac{e^{2} g_{A}^{2}}{192 \pi^{3} f_{\pi}^{2} M_{N}^{2}} \frac{1}{\mu^{2}\left(4-\mu^{2}\right)^{5 / 2}} \\
& \times\left[\sqrt { 4 - \mu ^ { 2 } } \left(6 \mu^{4}-52 \mu^{2}-3\left(\mu^{2}-4\right)^{2}\right.\right. \\
& \left.\times \mu^{2} \log \mu^{2}+16\right)+\mu\left(3 \mu^{6}-30 \mu^{4}\right. \\
& \left.\left.+94 \mu^{2}-40\right) \arccos \left(\frac{\mu^{2}}{2}-1\right)\right], \\
& \left.\left.+46 \mu^{2}-40\right) \arccos \left(\frac{\mu^{2}}{2}-1\right)\right], \\
\gamma_{3}^{(\pi \mathrm{N}, n)}= & -\frac{e^{2} g_{A}^{2}}{384 \pi^{3} f_{\pi}^{2} M_{N}^{2}} \frac{1}{\mu^{2}\left(4-\mu^{2}\right)^{5 / 2}} \\
& \times\left[\sqrt { 4 - \mu ^ { 2 } } \left(\mu^{2}\left(\mu^{2}-4\right)^{2} \log \mu^{2}\right.\right. \\
& \left.+2\left(\mu^{4}+10 \mu^{2}-8\right)\right)-\mu\left(\mu^{6}-10 \mu^{4}\right. \\
&
\end{aligned}
$$

$$
\begin{aligned}
\gamma_{4}^{(\pi \mathrm{N}, n)}= & \frac{e^{2} g_{A}^{2}}{384 \pi^{3} f_{\pi}^{2} M_{N}^{2}} \frac{1}{\mu^{2}\left(4-\mu^{2}\right)^{5 / 2}} \\
& \times\left[\sqrt { 4 - \mu ^ { 2 } } \left(-6 \mu^{4}+76 \mu^{2}+5\left(\mu^{2}-4\right)^{2}\right.\right. \\
& \left.\times \mu^{2} \log \mu^{2}-16\right)-\mu\left(5 \mu^{6}-50 \mu^{4}\right. \\
& \left.\left.+166 \mu^{2}-88\right) \arccos \left(\frac{\mu^{2}}{2}-1\right)\right]
\end{aligned}
$$

$$
\begin{aligned}
\alpha_{E 2}^{(\pi \mathrm{N}, n)}= & -\frac{3}{2 M_{\mathrm{N}}^{2}} \beta_{M 1}^{(\pi \mathrm{N}, n)}-\frac{e^{2} g_{A}^{2}}{1920 \pi^{3} f_{\pi}^{2} M_{N}^{3}} \frac{1}{\mu^{3}\left(4-\mu^{2}\right)^{7 / 2}} \\
& \times\left[\mu \sqrt { 4 - \mu ^ { 2 } } \left(-617 \mu^{6}+5796 \mu^{4}-19728 \mu^{2}\right.\right. \\
& \left.+570\left(\mu^{2}-4\right)^{3} \mu^{2} \log \mu+14144\right) \\
& -3\left(95 \mu^{10}-1330 \mu^{8}+6570 \mu^{6}-13320 \mu^{4}\right. \\
& \left.\left.+6816 \mu^{2}+896\right) \arccos \left(\frac{\mu^{2}}{2}-1\right)\right], \quad(\mathrm{C} 15)
\end{aligned}
$$$$
\begin{aligned}
\beta_{M 2}^{(\pi \mathrm{N}, n)}= & -\frac{3}{2 M_{\mathrm{N}}^{2}} \alpha_{E 1}^{(\pi \mathrm{N}, n)}+\frac{e^{2} g_{A}^{2}}{1920 \pi^{3} f_{\pi}^{2} M_{N}^{3}} \frac{1}{\mu^{3}\left(4-\mu^{2}\right)^{5 / 2}} \\
& \times\left[\mu \sqrt { 4 - \mu ^ { 2 } } \left(-523 \mu^{4}+3780 \mu^{2}\right.\right. \\
& \left.+570\left(\mu^{2}-4\right)^{2} \mu^{2} \log \mu-368\right) \\
& -3\left(95 \mu^{8}-950 \mu^{6}+2890 \mu^{4}-1400 \mu^{2}+96\right) \\
& \left.\times \arccos \left(\frac{\mu^{2}}{2}-1\right)\right] .
\end{aligned}
$$

$2 \pi \Delta$ loops

Our expressions for the $\pi \Delta$-loop contributions are sufficiently compact when we leave one of the integrations over the Feynman parameters undone. The integrands involve the following function of the Feynman parameter $x$ :

$D_{\Delta}(x)=x^{2}+(1+\delta)^{2}-x\left(2+2 \delta+\delta^{2}-\mu^{2}\right)$

where $\mu=m_{\pi} / M_{\mathrm{N}}, \delta=\Delta / M_{\mathrm{N}}$. Furthermore, the divergent parts of the polarisabilities, absorbed by higher-order contact terms, are renormalised according to the modified minimal subtraction $(\overline{\mathrm{MS}})$ scheme, by setting to 0 the factor arising in the dimensional regularisation:

$\Xi=\frac{2}{4-D}-\gamma_{E}+\log \frac{4 \pi \Lambda^{2}}{M_{\mathrm{N}}^{2}}$

with $D \simeq 4$ the number of dimensions, $\gamma_{E}$ the Euler constant, and $\Lambda$ the renormalisation scale.

With these definitions we obtain ${ }^{4}$

$$
\begin{aligned}
& \alpha_{E 1}^{(\pi \Delta)}=\frac{e^{2} h_{A}^{2} M_{\mathrm{N}}}{1728 \pi^{3} f_{\pi}^{2} M_{\Delta}^{2}} \\
& \quad \times\left[8 \delta+\frac{35}{3}+\int_{0}^{1} \mathrm{~d} x\left(\frac{18(x-1)^{2} x^{4}(x+\delta+1)}{D_{\Delta}^{2}(x)}\right.\right. \\
& \quad-\frac{x^{2}\left(120 x^{3}+4(27 \delta-16) x^{2}-(166 \delta+115) x+57(\delta+1)\right)}{D_{\Delta}(x)} \\
& \left.\left.+6 x(x(22 x+15 \delta-1)-12(\delta+1))\left[\Xi-\log D_{\Delta}(x)\right]\right)\right],
\end{aligned}
$$

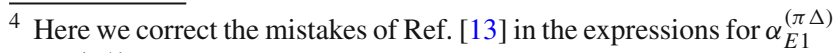
and $\beta_{M 1}^{(\pi \Delta)}$. The expressions for the other polarisabilities appear for the first time.
} 


$$
\begin{aligned}
& \beta_{M 1}^{(\pi \Delta)}=\frac{e^{2} h_{A}^{2} M_{\mathrm{N}}}{1728 \pi^{3} f_{\pi}^{2} M_{\Delta}^{2}}\left[-8 \delta-\frac{65}{6}\right. \\
& -\frac{x^{2}\left(21 x^{3}-4(3 \delta+5) x^{2}+(37 \delta+26) x-24(\delta+1)\right)}{D_{\Delta}(x)} \\
& +\int_{0}^{1} \mathrm{~d} x\left(\frac{\left(24 x^{2}-32 x+9\right) x^{2}(x+\delta+1)}{D_{\Delta}(x)}\right. \\
& \left.\left.+12(3 x-2) x^{2}\left[\Xi-\log D_{\Delta}(x)\right]\right)\right] \text {, } \\
& \left.\left.-6(5 x-4) x(2 x+3 \delta+3)\left[\Xi-\log D_{\Delta}(x)\right]\right)\right] \text {, } \\
& \gamma_{3}^{(\pi \Delta)}=\frac{e^{2} h_{A}^{2}}{1728 \pi^{3} f_{\pi}^{2} M_{\Delta}^{2}} \\
& \gamma_{1}^{(\pi \Delta)}=\frac{e^{2} h_{A}^{2}}{1728 \pi^{3} f_{\pi}^{2} M_{\Delta}^{2}}\left[2 \delta+\frac{7}{4}\right. \\
& +\int_{0}^{1} \mathrm{~d} x\left(\frac{8(x-1)^{3} x^{5}(x+\delta+1)}{D_{\Delta}^{3}(x)}\right. \\
& -\frac{2(x-1)^{2} x^{3}\left(22 x^{2}+8 \delta x+3 \delta+3\right)}{D_{\Delta}^{2}(x)} \\
& +\frac{(x-1) x^{2}\left(105 x^{2}-2(5 \delta+38) x+33(\delta+1)\right)}{D_{\Delta}(x)} \\
& +2 x(x(-16 x+9 \delta+21)-12(\delta+1)) \\
& \left.\left.\times\left[\Xi-\log D_{\Delta}(x)\right]\right)\right], \\
& \times\left[-\delta-\frac{2}{3}+\int_{0}^{1} \mathrm{~d} x\left(\frac{(x-1) x^{5}(x+\delta+1)}{D_{\Delta}^{2}(x)}\right.\right. \\
& -\frac{x^{2}\left(24 x^{3}+(2 \delta-11) x^{2}+(8 \delta-1) x-9(\delta+1)\right)}{2 D_{\Delta}(x)} \\
& +x(x(34 x-9 \delta-33)+12(\delta+1)) \\
& \left.\left.\times\left[\Xi-\log D_{\Delta}(x)\right]\right)\right], \\
& \gamma_{4}^{(\pi \Delta)}=\frac{e^{2} h_{A}^{2}}{1728 \pi^{3} f_{\pi}^{2} M_{\Delta}^{2}}\left[\delta+\frac{2}{3}+\int_{0}^{1} \mathrm{~d} x\right. \\
& \times\left(-\frac{\left(x^{3}+5 x^{2}-12 x+6\right) x^{3}(x+\delta+1)}{D_{\Delta}^{2}(x)}\right. \\
& +\frac{x^{2}\left(48 x^{3}-(22 \delta+59) x^{2}+(80 \delta+71) x-57(\delta+1)\right)}{2 D_{\Delta}(x)} \\
& +x(x(-34 x+9 \delta+33)-12(\delta+1)) \\
& \gamma_{2}^{(\pi \Delta)}=\frac{e^{2} h_{A}^{2}}{1728 \pi^{3} f_{\pi}^{2} M_{\Delta}^{2}} \\
& \times\left[\frac{5}{12}+\int_{0}^{1} \mathrm{~d} x\left(\frac{2\left(4 x^{2}-7 x+3\right) x^{3}(x+\delta+1)}{D_{\Delta}^{2}(x)}\right.\right. \\
& \left.\left.\times\left[\Xi-\log D_{\Delta}(x)\right]\right)\right] \text {, }
\end{aligned}
$$

$$
\begin{aligned}
& \alpha_{E 2}^{(\pi \Delta)}=\frac{e^{2} h_{A}^{2}}{17280 \pi^{3} f_{\pi}^{2} M_{\Delta}^{2} M_{\mathrm{N}}} \times\left[-\frac{325}{2}-120 \delta+\int_{0}^{1} \mathrm{~d} x\left(\frac{80(x-1)^{2} x^{5}(13 x-9)(x+\delta+1)}{D_{\Delta}^{3}(x)}\right.\right. \\
& -\frac{2 x^{3}\left(2235 x^{4}+(2095 \delta-2569) x^{3}-3(1518 \delta+563) x^{2}+15(199 \delta+167) x-570(\delta+1)\right)}{D_{\Delta}^{2}(x)} \\
& +\frac{15 x^{2}\left(201(\delta+1)+494 x^{3}+4(101 \delta-72) x^{2}-(608 \delta+407) x\right)}{D_{\Delta}(x)} \\
& \left.\left.-90 x(5 x-4)(2 x+3 \delta+3)\left[\Xi-\log D_{\Delta}(x)\right]\right)\right]-\frac{3}{2} \frac{\beta_{M 1}^{(\pi \Delta)}}{M_{\mathrm{N}}^{2}},
\end{aligned}
$$




$$
\begin{aligned}
& \beta_{M 2}^{(\pi \Delta)}=\frac{e^{2} h_{A}^{2}}{5760 \pi^{3} f_{\pi}^{2} M_{\Delta}^{2} M_{\mathrm{N}}} \times\left[\frac{175}{3}+40 \delta\right. \\
& +\int_{0}^{1} \mathrm{~d} x\left(\frac{4 x^{3}\left(75 x^{3}-146 x^{2}+80 x-15\right)(x+\delta+1)}{D_{\Delta}^{2}(x)}\right. \\
& -\frac{5 x^{2}\left(286 x^{3}+(320 \delta-84) x^{2}-5(94 \delta+71) x+153(\delta+1)\right)}{D_{\Delta}(x)} \\
& \left.\left.+30 x\left(22 x^{2}+(15 \delta-1) x-12(\delta+1)\right)\left[\Xi-\log D_{\Delta}(x)\right]\right)\right] \\
& -\frac{3}{2} \frac{\alpha_{E 1}^{(\pi \Delta)}}{M_{\mathrm{N}}^{2}} .
\end{aligned}
$$

\section{$3 \Delta$ excitation}

Introducing $M_{+}=M_{\mathrm{N}}+M_{\Delta}$ and recalling that $\Delta=$ $M_{\Delta}-M_{\mathrm{N}}$, the contribution of the Delta(1232) electromagnetic excitation to the dipole, spin and quadrupole polarisabilities of the proton are, respectively,

$$
\begin{aligned}
\alpha_{E 1}^{(\Delta)}= & -\frac{e^{2}}{4 \pi} \frac{2 g_{E}^{2}}{M_{+}^{3}}, \\
\beta_{M 1}^{(\Delta)}= & \frac{e^{2}}{4 \pi} \frac{2 g_{M}^{2}}{M_{+}^{2} \Delta}, \\
\gamma_{1}^{(\Delta)}= & \frac{e^{2}}{4 \pi} \frac{1}{M_{+}^{2} M_{\mathrm{N}}}\left(\frac{g_{M}^{2}}{\Delta}-\frac{2 g_{E} g_{M}}{M_{+}}+\frac{g_{E}^{2} M_{\Delta}}{M_{+}^{2}}\right), \\
\gamma_{2}^{(\Delta)}= & -\frac{e^{2}}{4 \pi} \frac{1}{M_{+}^{2} M_{\mathrm{N}}}\left(\frac{g_{M}^{2} M_{\Delta}}{\Delta^{2}}+\frac{g_{E} g_{M}}{\Delta}\right), \\
\gamma_{3}^{(\Delta)}= & -\frac{e^{2}}{4 \pi} \frac{1}{M_{+}^{2} M_{\mathrm{N}}}\left(\frac{g_{M}^{2}}{2 \Delta}-\frac{3 g_{E} g_{M}}{2 M_{+}}\right), \\
\gamma_{4}^{(\Delta)}= & \frac{e^{2}}{4 \pi} \frac{1}{M_{+}^{2} M_{\mathrm{N}}}\left(\frac{g_{M}^{2} M_{\Delta}}{\Delta^{2}}-\frac{g_{E} g_{M}}{2 \Delta}+\frac{g_{E}^{2}}{2 M_{+}}\right), \\
\alpha_{E 2}^{(\Delta)}= & -\frac{3}{2 M_{\mathrm{N}}^{2}} \beta_{M 1}^{(\Delta)}+\frac{e^{2}}{4 \pi} \frac{3}{M_{+}^{2} M_{\mathrm{N}}^{2}} \\
& \times\left(\frac{2 g_{M}^{2}}{\Delta}-\frac{g_{E} g_{M}}{M_{+}}+\frac{g_{E}^{2}\left(3 M_{\Delta} M_{+}+\Delta^{2}\right)}{M_{+}^{2} \Delta}\right), \\
\beta_{M 2}^{(\Delta)}= & -\frac{3}{2 M_{\mathrm{N}}^{2}} \alpha_{E 1}^{(\Delta)}-\frac{e^{2}}{4 \pi} \frac{3}{M_{+}^{2} M_{\mathrm{N}}^{2}} \\
& \times\left(\frac{g_{M}^{2}\left(3 M_{\Delta} \Delta+M_{+}^{2}\right)}{\Delta^{2} M_{+}}-\frac{g_{E} g_{M}}{\Delta}+\frac{2 g_{E}^{2}}{M_{+}}\right) .
\end{aligned}
$$

\section{Appendix D: Details of higher-order Delta self-energy}

For the benefit of future workers, we here provide the details of the corrections to the Delta self-energy which enter beyond the order to which we work in this paper, as discussed in Sect. 2.3 .
We write the Delta self-energy in the form:

$\Sigma(\not p)=\sigma(s)+\tau(s)\left(\not p-M_{\Delta}\right)$,

where the one-loop contributions with the intermediate nucleon or Delta are given by [32]

$$
\begin{aligned}
\sigma(s)_{N, \Delta}= & -\frac{C_{N, \Delta}}{2\left(8 \pi f_{\pi}\right)^{2}} \int_{0}^{1} \mathrm{~d} x\left(x M_{\Delta}+M_{N, \Delta}\right) \mathcal{M}_{N, \Delta}^{2} \\
& \times\left[-\Xi-1+\log \mathcal{M}_{N, \Delta}^{2}\right], \\
\tau(s)_{N, \Delta}= & -\frac{C_{N, \Delta}}{2\left(8 \pi f_{\pi}\right)^{2}} \int_{0}^{1} \mathrm{~d} x x \mathcal{M}_{N, \Delta}^{2} \\
& \times\left[-\Xi-1+\log \mathcal{M}_{N, \Delta}^{2}\right],
\end{aligned}
$$

with $\Xi$ the constant representing the ultraviolet divergence, $C_{N}=h_{A}^{2}, C_{\Delta}=\left(5 / 3 H_{A}\right)^{2}$, and

$\mathcal{M}_{N, \Delta}^{2}=m_{\pi}^{2} x+M_{N, \Delta}^{2}(1-x)-s x(1-x)$.

For the $\pi \Delta$ axial coupling we adopt the large- $N_{C}$ value $H_{A}=9 / 5 g_{A}$. The renormalised functions $\sigma_{R}(s)$ and $\tau_{R}(s)$ are obtained after two and one subtractions, respectively:

$$
\begin{aligned}
& \sigma_{R}(s)=\sigma(s)-\operatorname{Re} \sigma\left(M_{\Delta}^{2}\right)-\left(s-M_{\Delta}^{2}\right) \operatorname{Re} \sigma^{\prime}\left(M_{\Delta}^{2}\right), \\
& \tau_{R}(s)=\tau(s)-\operatorname{Re} \tau\left(M_{\Delta}^{2}\right) .
\end{aligned}
$$

The running Delta mass is then given by $M_{\Delta}(s)=M_{\Delta}+$ $\operatorname{Re} \sigma_{R}(s)$, while the Delta width is $\Gamma(s)=-2 \operatorname{Im} \sigma(s)$.

While $\sigma_{R}(s)$ is finite, $\tau_{R}(s)$ still contains a divergent piece which is to be renormalised by an appropriate low-energy constant. In the absence of further information on such a constant, we neglect it and merely set $\Xi=0$. Note that the expressions for $\sigma(s)$ and $\tau(s)$ above do not contain the extra factor of $s / M_{\Delta}^{2}$ seen in Ref. [32]. This is due to the fact that each of the leading $\gamma N \Delta$ vertices in the first graph in Fig. 3 brings in an extra Delta momentum giving a total factor of $s$ (see Ref. [10]). When the self-energy graphs are resummed in the intermediate state, the factor $s / M_{\Delta}^{2}$ is taken out from the self-energy in order to be restored in front of the total result, hence this factor is absent in the expressions we use here.

We also give here the dispersion integral that simplifies the calculation of the running couplings $g_{M, E}(s)$. Namely, the running due to the leading loop corrections can be calculated from the already employed imaginary parts of these corrections by the once-subtracted integral

$$
\begin{aligned}
\operatorname{Re} g_{M, E}(s)= & \operatorname{Re} g_{M, E}\left(M_{\Delta}^{2}\right)+\left(s-M_{\Delta}^{2}\right) \\
& \times \int_{\left(M_{N}+m_{\pi}\right)^{2}}^{\infty} \frac{\mathrm{d} s}{\pi} \frac{\operatorname{Im} g_{M, E}\left(s^{\prime}\right)}{\left(s^{\prime}-M_{\Delta}^{2}\right)\left(s^{\prime}-s\right)},
\end{aligned}
$$


where the integral is understood as the Cauchy principal value; note the explicit $\left(s-M_{\Delta}^{2}\right)$ factor.

\section{References}

1. H.W. Grießhammer, J.A. McGovern, D.R. Phillips, G. Feldman, Prog. Part. Nucl. Phys. 67, 841 (2012). arXiv: 1203.6834 [nucl-th]

2. B.R. Holstein, S. Scherer, Ann. Rev. Nucl. Part. Sci. 64, 51 (2014). arXiv:1401.0140 [hep-ph]

3. F. Hagelstein, R. Miskimen, V. Pascalutsa, to appear in Prog. Part. Nucl. Phys. arXiv:1512.03765 [nucl-th]

4. S. Weinberg, Physica A 96, 327 (1979)

5. J. Gasser, H. Leutwyler, Ann. Phys. 158, 142 (1984)

6. J. Gasser, M.E. Sainio, A. Svarc, Nucl. Phys. B 307, 779 (1988)

7. J.A. McGovern, Phys. Rev. C 63, 064608 (2001) [Erratum-ibid. C 66, 039902 (2002)]. arXiv:nucl-th/0101057

8. S.R. Beane, M. Malheiro, J.A. McGovern, D.R. Phillips, U. van Kolck, Phys. Lett. B 567, 200 (2003) [Erratum-ibid. B 607, 320 (2005)]. arXiv:nucl-th/0209002

9. S.R. Beane, M. Malheiro, J.A. McGovern, D.R. Phillips, U. van Kolck, Nucl. Phys. A 747, 311 (2005). arXiv:nucl-th/0403088

10. V. Pascalutsa, D.R. Phillips, Phys. Rev. C 67, 055202 (2003). arXiv:nucl-th/0212024

11. J.A. McGovern, D.R. Phillips, H.W. Grießhammer, Eur. Phys. J. A 49, 12 (2013). arXiv:1210.4104 [nucl-th]

12. V. Lensky, V. Pascalutsa, Pisma Zh. Eksp. Teor. Fiz. 89, 127 (2009) [JETP Lett. 89, 108 (2009)]. arXiv:0803.4115 [nucl-th]

13. V. Lensky, V. Pascalutsa, Eur. Phys. J. C 65, 195 (2010). arXiv:0907.0451 [hep-ph]

14. V. Lensky, J.A. McGovern, Phys. Rev. C 89, 032202 (2014). arXiv:1401.3320 [nucl-th]

15. V. Lensky, J.A. McGovern, D.R. Phillips, V. Pascalutsa, Phys. Rev. C 86, 048201 (2012). arXiv:1208.4559 [nucl-th]

16. D. Hornidge, E.J. Downie, J.R.M. Annand, I.J.D. MacGregor, Mainz Proposal A2-11/09 (2009)

17. H.R. Weller, M.W. Ahmed, H. Gao, W. Tornow, Y.K. Wu, M. Gai, R. Miskimen, Prog. Part. Nucl. Phys. 62, 257 (2009)

18. P.P. Martel et al. [A2 Collaboration], Phys. Rev. Lett. 114, 112501 (2015). arXiv:1408.1576 [nucl-ex]

19. V. Bernard, N. Kaiser, U.-G. Meißner, Phys. Rev. Lett. 67, 1515$1518(1991)$

20. V. Bernard, N. Kaiser, A. Schmidt, U.-G. Meißner, Phys. Lett. B 319, 269 (1993). arXiv:hep-ph/9309211

21. V. Bernard, N. Kaiser, U.-G. Meißner, Int. J. Mod. Phys. E 4, 193 (1995). arXiv:hep-ph/9501384

22. T.R. Hemmert, B.R. Holstein, J. Kambor, G. Knochlein, Phys. Rev. D 57, 5746 (1998). arXiv:nucl-th/9709063

23. B.R. Holstein, D. Drechsel, B. Pasquini, M. Vanderhaeghen, Phys. Rev. C 61, 034316 (2000). arXiv:hep-ph/9910427

24. X.D. Ji, C.W. Kao, J. Osborne, Phys. Rev. D 61, 074003 (2000). arXiv:hep-ph/9908526

25. K.B. Vijaya Kumar, J.A. McGovern, M.C. Birse, Phys. Lett. B 479, 167, (2000). arXiv:hep-ph/0002133

26. G.C. Gellas, T.R. Hemmert, U.-G. Meißner, Phys. Rev. Lett. 85, 14 (2000). arXiv:nucl-th/0002027

27. V. Bernard, T.R. Hemmert, U.-G. Meißner, Phys. Rev. D 67, 076008 (2003). arXiv:hep-ph/0212033

28. V. Bernard, E. Epelbaum, H. Krebs, U.G. Meissner, Phys. Rev. D 87, 5, 054032 (2013). arXiv:1209.2523 [hep-ph]

29. A.H. Blin, T. Gutsche, T. Ledwig, V.E. Lyubovitskij, arXiv: 1509.00955 [hep-ph]

30. A.M. Gasparyan, M.F.M. Lutz, B. Pasquini, Nucl. Phys. A 866, 79 (2011). arXiv:1102.3375 [hep-ph]
31. V. Pascalutsa, M. Vanderhaeghen, S.N. Yang, Phys. Rep. 437, 125 (2007). arXiv:hep-ph/0609004

32. V. Pascalutsa, M. Vanderhaeghen, Phys. Rev. D 73, 034003 (2006). arXiv:hep-ph/0512244

33. K.A. Olive et al. [Particle Data Group Collaboration], Chin. Phys. C 38, 090001 (2014)

34. D. Babusci, G. Giordano, A.I. L'vov, G. Matone, A.M. Nathan. Phys. Rev. C 58, 1013 (1998). arXiv:hep-ph/9803347

35. B. Pasquini, P. Pedroni, D. Drechsel, Phys. Lett. B 687, 160 (2010). arXiv: 1001.4230 [hep-ph]

36. A.M. Baldin, Nucl. Phys. 18, 310 (1960)

37. V. Olmos de León et al., Eur. Phys. J. A 10, 207 (2001)

38. R.P. Hildebrandt, H.W. Grießhammer, T.R. Hemmert, B. Pasquini, Eur. Phys. J. A 20, 293 (2004). arXiv:nucl-th/0307070

39. B. Pasquini, private communications

40. O. Gryniuk, F. Hagelstein, V. Pascalutsa, Phys. Rev. D 92, 074031 (2015). arXiv:1508.07952 [nucl-th]

41. B. Pasquini, D. Drechsel, M. Vanderhaeghen, Phys. Rev. C 76, 015203 (2007). arXiv:0705.0282 [hep-ph]

42. H. W. Grießhammer, J. A. McGovern, D. R. Phillips. arXiv:1511.01952 [nucl-th]

43. D. Drechsel, B. Pasquini, M. Vanderhaeghen, Phys. Rep. 378, 99 (2003). arXiv:hep-ph/0212124

44. K. Kossert et al., Eur. Phys. J. A 16, 259 (2003). arXiv:nucl-ex/0210020

45. L.S. Myers et al. [COMPTON@MAX-lab Collaboration], Phys. Rev. Lett. 113, 262506 (2014). arXiv:1409.3705 [nucl-ex]

46. M.I. Levchuk, A.I. L'vov, Nucl. Phys. A 674, 449 (2000). arXiv:nucl-th/9909066

47. M. Schumacher, M.I. Levchuk, Nucl. Phys. A 858, 48 (2011). arXiv:1104.3721 [hep-ph]

48. E. Epelbaum, H. Krebs, U.-G. Meißner, Eur. Phys. J. A 51(5), 53 (2015). arXiv:1412.0142 [nucl-th]

49. E. Epelbaum, H. Krebs, U.G. Meißner, Phys. Rev. Lett. 115, 122301 (2015). arXiv:1412.4623 [nucl-th]

50. R.J. Furnstahl, N. Klco, D.R. Phillips, S. Wesolowski, arXiv:1506.01343 [nucl-th]

51. I. Guiasu, E.E. Radescu, Ann. Phys. 120, 145 (1979)

52. I. Guiasu, E.E. Radescu, Ann. Phys. 122, 436 (1979)

53. H.W. Grießhammer, T.R. Hemmert, Phys. Rev. C 65, 045207 (2002). arXiv:nucl-th/0110006

54. R.P. Hildebrandt, H.W. Grießhammer, T.R. Hemmert, Eur. Phys. J. A 20, 329 (2004). arXiv:nucl-th/0308054

55. A. Aleksejevs, S. Barkanova, Nucl. Phys. Proc. Suppl. 245, 17 (2013). arXiv:1309.3313 [hep-ph]

56. A. Aleksejevs, private communication

57. J. McGovern, H. Grießhammer, D. Phillips, Workshop on Compton scattering off Protons and Light Nuclei, ECT* Trento (2013). http:// www.ectstar.eu/sites/www.ectstar.eu/files/talks/mcgovern.pdf

58. D. Drechsel, S.S. Kamalov, L. Tiator, Eur. Phys. J. A 34, 69 (2007). arXiv:0710.0306 [nucl-th]

59. R.P. Hildebrandt, PhD Thesis, T. U. München (2005). arXiv:nucl-th/0512064

60. G. Blanpied, M. Blecher, A. Caracappa, R. Deininger, C. Djalali, G. Giordano, K. Hicks, S. Hoblit et al., Phys. Rev. C 64, 025203 (2001)

61. C. Collicott, PhD Thesis, Dalhousie University (2015)

62. O. Grinyuk, F. Hagelstein, N. Krupina, V. Pascalutsa, in preparation

63. J.A.M. Vermaseren, arXiv:math-ph/0010025

64. T. Hahn, M. Perez-Victoria, Comput. Phys. Commun. 118, 153 (1999). arXiv:hep-ph/9807565 